TRANSACTIONS OF THE

AMERICAN MATHEMATICAL SOCIETY

Volume 348, Number 10, October 1996

\title{
A NEW UNCOUNTABLY CATEGORICAL GROUP
}

\author{
ANDREAS BAUDISCH
}

\begin{abstract}
We construct an uncountably categorical group with a geometry that is not locally modular. It is not possible to interpret a field in this group. We show the group is CM-trivial.
\end{abstract}

\section{INTRODUCTION}

Let $T$ be a countable complete first order theory. $T$ is called $\lambda$-categorical if all models of $T$ of cardinality $\lambda$ are isomorphic. In [15] M. D. Morley proved J. Łoś's conjecture that a theory $T$ that is categorical in some uncountable power is categorical in every uncountable power. These uncountably categorical theories are the simplest case of a theory $T$ that allows the classification of its models in the sense of S. Shelah [22]. The main examples of such theories are the theories of algebraically closed fields of a given characteristic. The classification of the models of an uncountably categorical theory is given by the unique size of a "transcendence basis" of the models, similar to the classification of algebraically closed fields by Steinitz [1].

For any uncountably categorical theory $T$ one can find a formula $\phi(x)$ (with auxiliary parameters) which is strongly minimal in the sense that the set defined by $\phi$ in any model of $T$ cannot be split into two infinite definable sets. The appropriate generalization of the notion of "transcendence degree" involves a combinatorial geometry with the Steinitz exchange property, which is defined on any strongly minimal set.

Let $D$ be any set. Assume that $c l$ is an operator from the set of finite subsets of $D$ into the set of subsets of $D$. We say that $c l$ defines a pregeometry on $D$ if the following conditions are fulfilled. Let $a, b$ be elements of $D$ and $A, B$ be finite subsets of $D$ :

i) $A \subseteq \operatorname{cl}(A)$.

ii) $A \subseteq B$ implies $c l(A) \subseteq \operatorname{cl}(B)$.

iii) $\operatorname{cl}(\operatorname{cl}(A))=\operatorname{cl}(A)$.

iv) If $a \in \operatorname{cl}(A \cup\{b\}) \backslash \operatorname{cl}(A)$, then $b \in \operatorname{cl}(A \cup\{a\})$.

A pregeometry is called a geometry if $c l(\{a\})=\{a\}$ for each point a. One may pass from a pregeometry to a geometry by removing $c l(\emptyset)$ and taking the closures of the remaining points as the points of a geometry. There is a natural geometry on any strongly minimal set in which $\operatorname{cl}(A)$ is the union of all finite $A$-definable sets; this is called the algebraic closure in the sense of model theory. Hence every

Received by the editors October 26, 1993 and, in revised form, March 10, 1995.

1991 Mathematics Subject Classification. Primary 03C35, 03C45, 03C60.

Heisenberg-Stipendiat der Deutschen Forschungsgemeinschaft.

(C)1996 American Mathematical Society 
strongly minimal formula of $T$ defines a geometry for $T$, and all the geometries obtained in this way are isomorphic up to finite localizations. We speak of the geometry of $T$.

Let us mention the following examples:

1) The theory of the successor function on the naturals. The corresponding geometry is disintegrated, i.e.: $a \in \operatorname{cl}(A)$ if and only if $a \in A$.

2) The theory of a vector space where the language contains,,+- 0 and a unary function for each element of the base field, corresponding to scalar multiplication by that element. The corresponding geometry is the projective geometry. This geometry is modular: If $X$ and $Y$ are closed sets, then the geometrical dimension $d$ fulfills the following equation:

$$
d(X)+d(Y)=d(X \cap Y)+d(X \cup Y) .
$$

3) The theory of an algebraically closed field. The corresponding geometry is not locally modular. That means even a localization of the geometry at a finite set is not modular.

First B. Zil'ber [25] tried to classify uncountably categorical theories $T$ according to their geometries. He proved:

- If the geometry is disintegrated, then no group is interpretable in $T$.

Then E. Hrushovski showed:

- If the geometry is not disintegrated but still locally modular, then a group but no field is interpretable in $T$.

B. Zil'ber conjectured that in the the remaining case (the geometry is not locally modular) a field is interpretable in T. In $1988 \mathrm{E}$. Hrushovski [12] found a counterexample of an uncountably categorical theory with a non-locally-modular geometry and without the possibility of interpreting a group. The aim of this paper is to construct a pure group with an uncountably categorical theory and a non-locallymodular geometry that does not allow the interpretation of a field. "Pure group" means that we work in the language of group theory only.

There are only two possibilities in searching for such a group $G$. Either $G$ is a nilpotent group of bounded exponent, or a quotient of definable subgroups of $G$ is a counterexample to the Cherlin-Zil'ber Conjecture that every simple group of finite Morley rank is an algebraic group over an algebraically closed field. This is shown in section 2 . We consider nilpotent groups of class 2 with exponent $p$ where $p$ is a prime greater than 2 . Note that an uncountably categorical pure group has a locally modular geometry if and only if it is abelian by finite [13].

We transfer the construction of a Hrushovski-geometry in relational languages [12] (see also [10]) to the case of a pure group. The most difficult part is the proof of the corresponding Amalgamation Theorems.

The final group is obtained by the method of successive amalgamation as introduced by R. Fraïssé [9]. We want to amalgamate finite nilpotent groups of class 2 of exponent $p>2$ with the additional property that the commutator subgroup is the center. But it is more convenient to work in an auxiliary category $\mathcal{S}$ whose objects are vector spaces $V$ over a finite field $F_{p}$ of order p together with a distinguished subspace $N(V)$ of the exterior square $\wedge^{2} V$ (see section 3). There is a functorial correspondence between the groups above and $\mathcal{S}$ that is 1-1 at the level of isomorphism types of objects. We lose some morphisms. 
In the relational case E. Hrushovski defined a function

$$
\delta(A)=\text { number of elements - number of relations }
$$

for all finite substructures $A$ of the structure under consideration. The corresponding function in our construction is

$$
\delta(H)=\operatorname{dim}(H)-\operatorname{dim}(N(H))
$$

where $H$ is a finite subspace of the underlying vectorspace $V$ of a structure in $\mathcal{S}$ and $\operatorname{dim}$ is the linear dimension. Note that by definition $N(H)=N(V) \cap \wedge^{2} H$. We only consider structures in $\mathcal{S}$ that fulfill the following condition:

( $\Sigma 3)$ : For all finite subspaces $H$ of $V$ we have $\delta(H) \geq 3$ if $\operatorname{dim}(H) \geq 3$, and $\delta(H)=\operatorname{dim}(H)$ otherwise.

As in the relational case, $\delta$ can be used to define a pregeometry $c l$ on $V$ provided $(\Sigma 3)$ is fulfilled. We give a short description of the definition of $\mathrm{cl}$. A finite subspace $H$ of $V$ is called selfsufficient if $\delta(K) \geq \delta(H)$ for all finite $K$ with $H \subseteq K \subseteq V$. Every finite subspace $H$ is contained in the smallest finite selfsufficient subspace $C S S(H)$ that is contained in every selfsufficient extension of $H$. Then we can define the desired pregeometry by

$$
a \in \operatorname{cl}\left(a_{1}, \ldots, a_{n}\right) \quad \text { iff } \quad \delta\left(C S S\left(\left\langle\left\{a_{1}, \ldots, a_{n}\right\}\right\rangle\right)\right)=\delta\left(C S S\left(\left\langle\left\{a, a_{1}, \ldots, a_{n}\right\}\right\rangle\right)\right) .
$$

The details of the definitions of all notions above are given in section 3 .

Now assume that $A$ is a subspace of $B$ and both are finite subspaces of $V$ in some $\mathcal{S}$-structure. Then $B$ is called a minimal extension of $A$ if $\delta(A)=\delta(B)$ and $\delta(K)>\delta(A)$ for all $K$ with $A \subseteq K \subseteq B$ and $A \neq K \neq B$. To describe the amalgamation process let $A, B$, and $C$ be finite structures in $\mathcal{S}$ such that $A$ is a common substructure of $B$ and $C$, and $B$ is a minimal extension of $A$. If $A$ is selfsufficient in $C$ and $B$ is not realized in $C$ over $A$, then we replace $C$ by the following free amalgam $D=B \star_{A} C$ of $B$ and $C$ over $A$ : The underlying vectorspace $D$ is $B \oplus_{A} C$, and $N(D)$ is the subspace $N(B)+N(C)$ of $\wedge^{2} D$. The First Amalgamation Theorem in section 4 ensures that $D$ satisfies ( $\Sigma 3$ ) again, provided that $A, B$, and $C$ fulfil ( $\Sigma 3)$. Section 5 contains some preparations for the argument in section 6 .

Let $(M, N(M))$ be the final $\mathcal{S}$-structure produced by the amalgamation process described above. Let $G$ be the corresponding group and let $\bar{G}$ be $G / Z(G)$ where $Z(G)$ is the center of $G$. Then we can identify the vector spaces $M$ and $\bar{G}$. We have to modify the construction in such a way that we get $c l=a c l$ on $\bar{G}$, where $c l$ is the pregeometry given by $(M, N(M))$ and acl is the algebraic closure on $\bar{G}$ given by the group $G$. For this it is sufficient that the number of realizations of a minimal extension $K$ over $H$ has a bound which depends only on $\operatorname{dim}(K)$. The complicated condition $(\Sigma 4)$ implies such a bound. In section 6 we prove that $(\Sigma 4)$ is preserved by the described amalgamation (Second Amalgamation Theorem). Then we can show that the theory $\Sigma$ of the corresponding group will be uncountably categorical and its geometry will be given by $c l$ on $M$. In section 7 the construction of the group and the final results are given apart from the non-interpretability of a field. This will be done in section 8. We show CM-triviality of the theory $\Sigma$. CM-triviality was introduced by E. Hrushovski in [12]. This implies the desired non-interpretability of a field. In the first version of this paper we proved directly that every group $H$ that we can interpret in our group is nilpotent by finite and bounded. 
I am very grateful to Paul Eklof and Ali Nesin for an inspiring time at the University of California at Irvine in winter and spring 1991 when I started this work. Furthermore, I gratefully acknowledge helpful suggestions of Greg Cherlin, Ehud Hrushovski, Anand Pillay, and Martin Ziegler.

\section{On Groups of Finite Morley RANK}

If $G$ is a group, then let $[X, Y]$ be the set $\{[a, b]: a \in X, b \in Y\}$. For subsets $X \subseteq$ $G$ let $\langle X\rangle$ be the subgroup generated by $X$. Hence we can define the commutator subgroup $G^{\prime}$ as $\langle[X, Y]\rangle$. We use $Z(G)$ to denote the center of $G$.

Uncountably categorical theories are $\omega$-stable of finite Morley rank. A group is called connected if it contains no proper definable subgroup of finite index. An $\omega$ stable group $G$ contains a definable connected normal subgroup $G^{0}$ of finite index. It is called the connected component of $G$.

There is a well-known conjecture of G. Cherlin and B. Zil'ber that every simple infinite group of finite Morley rank is an algebraic group over an algebraically closed field. Combining a number of known results, we obtain the following:

Theorem 2.1. Let $G$ be a group of finite Morley rank that does not allow the interpretation of an infinite field. Either we get a counterexample to the Cherlin Zil'ber-Conjecture as a quotient of definable subgroups of $G$, or $G^{0}=A * B$ where $A$ is Abelian and $B$ is a nilpotent definable subgroup of bounded exponent. (Here * denotes a central product.)

Proof. Even in the superstable case there are definable subgroups $H_{0} \triangleleft H_{1} \triangleleft \ldots \triangleleft$ $H_{r}=G^{0}$ of $G$ where $H_{i+1} / H_{i}$ is infinite and furthermore either abelian or simple over a finite center. In the case of finite Morley rank this follows from Zil'ber's Indecomposability Theorem ([23], see [7]). The proof for superstable groups is found in [5]. Hence we either have an infinite simple group as a quotient of definable subgroups of $G$, or else $G^{0}$ is soluble. We need the following result of Zil'ber [24]:

Theorem 2.2. In every connected soluble non-nilpotent group of finite Morley rank it is possible to interpret a field.

In a simple algebraic group over an algebraically closed field one can find a definable connected soluble non-nilpotent subgroup. By Theorem 2.2 a field is definable. Hence if we get a simple group above, then it is a counterexample to the Cherlin-Zil'ber Conjecture. Otherwise we know that $G^{0}$ is soluble. By Theorem 2.2 again we obtain that $G^{0}$ is nilpotent.

Note that B. Zil'ber [26] has shown that every torsion-free nilpotent uncountably categorical group is an algebraic group over an algebraically closed field. C. Grünenwald and F. Haug obtained the following (Theorem 2.3 in [11] ):

Theorem 2.3. Let $G$ be a non-Abelian nilpotent stable group. Assume that the commutator subgroup $G^{\prime}$ is torsion-free. Then an infinite field of characteristic 0 is interpretable.

Furthermore we need the following result of A. Nesin [16]:

Theorem 2.4. Let $G$ be an $\omega$-stable nilpotent group. Then there are definable characteristic subgroups $D$ and $B$ such that $D$ is divisible, $B$ has finite exponent, $G=D B$, and $[D, B]=\langle 1\rangle$. D can be decomposed as $D=D^{+} \oplus F$, where $D^{+}$is the central subgroup of torsion elements of $D$ and $F$ is a torsion-free subgroup. 
Now our group $G^{0}$ has the structure described by Theorem 2.4. If $D$ is Abelian, then $D$ is central and the assertion follows. Otherwise consider the definable subgroup $D$. Then $D^{\prime}=F^{\prime} . F^{\prime}$ is torsion-free and non-trivial. By Theorem 2.3 it is possible to interpret a field, a contradiction.

Theorem 2.1 gives the motivation to consider only groups $G$, that satisfy the following property:

$(\Sigma 1): G$ is nilpotent of class 2 and of exponent $p$, where $p$ is a fixed prime greater than 2.

Let $F_{c}(p, \kappa)$ be the free group in the variety of nilpotent groups of class $c$ and of exponent $p(p>c) . \kappa$ is the number of free generators. Let $\kappa$ be infinite. In [2] it is shown that $F_{c}(p, \kappa)$ has an $\omega$-stable theory with $c$ dimensions. Thus $F_{2}(p, \kappa)$ is not so far from the group we desire; but this group has infinite Morley-rank. The reason for its two dimensions and the infinite Morley-rank is that there is no integer $n$ such that every element of the commutator subgroup $G^{\prime}$ can be presented as a product of at most $n$ commutators. In a group $G$ of finite Morley-rank we get, as a consequence of B. Zil'ber's Indecomposability Theorem, that there are $g_{1}, \ldots, g_{k} \in G$ such that

$$
G^{\prime}=\left[g_{1}, G\right]\left[g_{2}, G\right] \ldots\left[g_{k}, G\right] .
$$

Our final group will satisfy this condition in a very strong form:

( $\Sigma 2): \forall z \in Z(G) \forall x \notin Z(G) \exists y(z=[x, y])$.

All the groups $G$ considered here will satisfy $(\Sigma 1)$ and a weak form of $(\Sigma 2)$ :

$(\Sigma 2)^{w}: Z(G)=G^{\prime}$.

Note however that this condition is not elementary.

\section{The Geometry}

As above all the groups $G$ considered here will fulfill $(\Sigma 1)$ and $(\Sigma 2)^{w}$. Let $\bar{G}$ be $G / Z(G)$. If $G$ satisfies $(\Sigma 1)$ and $(\Sigma 2)^{w}$, then $[x, y]$ defines an alternating bilinear map of $\bar{G} \times \bar{G}$ into $Z(G)$ such that $[\bar{G}, \bar{G}]$ generates $Z(G)$. We will show that $G$ is determined up to isomorphism by this bilinear map, using the fact that $\bar{G}$ and $Z(G)$ are vector spaces over the field $F_{p}$ with $p$ elements.

Lemma 3.1. Let $G$ and $H$ be groups with the properties $(\Sigma 1)$ and $(\Sigma 2)^{w}$. Let $\bar{G}=G / Z(G), \bar{H}=H / Z(H)$, and let $[,]_{G},[,]_{H}$ be the corresponding commutator maps. Assume there is an isomorphism $f^{B}:\left(\bar{G}, Z(G) ;[,]_{G}\right) \simeq\left(\bar{H}, Z(H) ;[,]_{H}\right)$. Let $\left\{a_{0}, a_{1}, \ldots\right\}$ be a basis of the vector space $\bar{G}$ and choose $c_{i} \in G$ in the coset modulo $Z(G)$ corresponding to $a_{i}$. Let $f\left(c_{i}\right)$ be any element in $H$ in the coset modulo $Z(H)$ corresponding to $f^{B}\left(a_{i}\right)$.

Then $f^{B}$ extends to an isomorphism $f$ of $G$ onto $H$.

Proof. $G$ can be reconstructed from $\left(\bar{G}, Z(G) ;[,]_{G}\right)$ in the following way: Let $G^{*}$ be the following group. The elements are tuples $\left(\sum_{\alpha} r_{\alpha} a_{\alpha}, z\right)$, where $z \in Z(G)$ and $r_{\alpha}=0$ up to finitely many $\alpha$. The group multiplication is defined by

$$
\left(\sum_{\alpha} r_{\alpha} a_{\alpha}, z_{1}\right) \circ\left(\sum_{\alpha} s_{\alpha} a_{\alpha}, z_{2}\right)=\left(\sum_{\alpha}\left(r_{\alpha}+s_{\alpha}\right) a_{\alpha}, z_{1}+z_{2}+\sum_{\beta<\alpha} r_{\alpha} s_{\beta}\left[a_{\alpha}, a_{\beta}\right]\right) .
$$

Then $G$ and $G^{*}$ are isomorphic. To prove this we write elements of $G$ uniquely as $\prod_{\alpha} c_{\alpha}^{r_{\alpha}} z$, where $z \in Z(G)$ and $r_{\alpha}=0$ up to finitely many $\alpha . g\left(\prod_{\alpha} c_{\alpha}^{r_{\alpha}} z\right)=$ 
$\left(\sum_{\alpha} r_{\alpha} g_{\alpha}, z\right)$ gives the desired isomorphism of $G$ and $G^{*}$ with $g\left(c_{i}\right)=\left(a_{i}, 0\right)$. Analogously we obtain an isomorphism $h$ of $H$ onto $H^{*}$ with $h\left(f\left(c_{i}\right)\right)=\left(f^{B}\left(a_{i}\right), 0\right)$. If $f^{+}$is the isomorphism of $G^{*}$ onto $H^{*}$ given by $f^{B}$, then $f=h^{-1} f^{+} g$ has the desired properties.

Let $\mathcal{G}$ be the category of all groups that satisfy $(\Sigma 1)$ and $(\Sigma 2)^{w}$ with group monomorphisms as morphisms. Let $\mathcal{B}$ be the category of all alternating bilinear maps $\beta: V \times V \rightarrow W$, where $V$ and $W$ are vector spaces over the field $F_{p}$, and the image of $\beta$ generates $W$ (this is an analog of condition $\left.(\Sigma 2)^{w}\right)$. The morphisms from $\left(V_{1}, W_{1} ; \beta_{1}\right)$ to $\left(V_{2}, W_{2} ; \beta_{2}\right)$ are pairs $(f, g)$ with $f: V_{1} \rightarrow V_{2}, g: W_{1} \rightarrow W_{2}$ so that $\beta_{2} f=g \beta_{1}$. The correspondence between groups $G$ in $\mathcal{G}$ and the associated structures $\left(\bar{G}, Z(G) ;[,]_{G}\right)$ in $\mathcal{B}$ rise to a functor from $\mathcal{G}$ onto $\mathcal{B}$; this correspondence is 1-1 at the level of objects, by the proof of Lemma 3.1. (Lemma 3.1 does not say that the map is onto, but the proof shows it.)

For every vector space $V$ there is a "free bilinear map over $V$ ", namely $\wedge$ : $V \times V \rightarrow \wedge^{2} V$ in $\mathcal{B}$, characterized by the following universal property:

$(\wedge)$ : For every alternating bilinear map $\beta: V \times V \rightarrow W$ there is a unique linear $f_{\beta}: \wedge^{2} V \rightarrow W$ such that $f_{\beta} \wedge=\beta$.

The vector space $\wedge^{2} V$ (equipped with the associated bilinear map $\wedge$ ) is called the exterior square of $V$ (cf. [14]). We denote the kernel of $f_{\beta}$ on $\wedge^{2} V$ by $N(\beta)$, or more commonly, abusing the notation, by $N(V)$. For any structure $(V, W ; \beta)$ in $\mathcal{B}$, there is a canonical isomorphism $(V, W ; \beta) \simeq\left(V, \wedge^{2} V / N(\beta) ; \pi\right)$ where $\pi$ is the composition of the canonical maps $V \times V \rightarrow \wedge^{2} V \rightarrow \wedge^{2} V / N(\beta)$. These considerations lead to the introduction of another category $\mathcal{S}$ equivalent to $\mathcal{B}$. The objects of $\mathcal{S}$ are pairs $(V, N)$ with $N \subseteq \wedge^{2} V$ and the maps $f:\left(V_{1}, N_{1}\right) \rightarrow\left(V_{2}, N_{2}\right)$ such that the induced map $\wedge^{2} f$ between the exterior squares satisfies: $\wedge^{2} f\left[N_{1}\right]=\wedge^{2} f\left[V_{1}\right] \cap N_{2}$. The main work in this paper is done in $\mathcal{S}$. Let $(M, N(M))$ be an element of $\mathcal{S}$. Often we write $M$ only. For $H \leq M$ we let $N(H)=\wedge^{2} H \cap N(M)$, where of course $\wedge^{2} H$ is canonically identified with a subspace of $\wedge^{2} M$. Note that $(H, N(H)) \in \mathcal{S}$. By the equivalence of the categories $\mathcal{B}$ and $\mathcal{S}$ Lemma 3.1 implies the following:

Corollary 3.2. Let $G$ and $H$ be groups in $\mathcal{G}$. Assume there is an isomorphism $f_{0}$ of a subgroup $G_{0} \in \mathcal{G}$ of $G$ onto a subgroup $H_{0} \in \mathcal{G}$ of $H$. Let $M$ and $N$ be $\mathcal{S}$-structures corresponding to $G$ and $H$ respectively. Let $M_{0}$ be the substructure of $M$ corresponding to $G_{0}$ and let $N_{0}$ be the substructure of $N$ corresponding to $H_{0}$. Assume there is an $\mathcal{S}$-isomorphism $g$ of $M$ onto $N$ such that the restriction of $g$ to $M_{0}$ is the $\mathcal{S}$-isomorphism induced by $f_{0}$. Then there is an isomorphism $f$ of $G$ onto $H$ that extends $f_{0}$ and induces the given $\mathcal{S}$-isomorphism $g$ of $M$ onto $N$.

With $M$ fixed, we let $\delta$ be the function defined on finite dimensional subspaces $H$ of $M$ by $\delta(H)=\operatorname{dim}(H)-\operatorname{dim}(N(H))$. We are interested in groups with relatively few relations. First we formulate this requirement for $\mathcal{S}$ :

$(\Sigma 3)$ : Let $(M, N(M))$ be an element of $\mathcal{S}$. For every finite subspace $H$ of $M$, $\delta(H) \geq \min \{3, \operatorname{dim}(H)\}$.

Sometimes a weaker condition is sufficient:

$(\Sigma 3)^{0}$ : For every finite subspace $H$ of $M, \delta(H) \geq 0$.

Let $G$ be a group in $\mathcal{G}$. We say that $G$ fulfills $(\Sigma 3)$ if the corresponding structure in $\mathcal{S}$ satisfies $(\Sigma 3)$. In the language of groups we can formulate this by the following statement. It is possible to express this by a set of elementary sentences. 


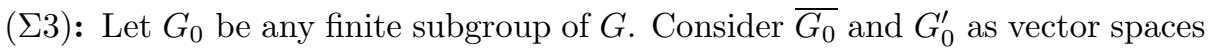
over $F_{p}$. If $\operatorname{dim}\left(\overline{G_{0}}\right)=n$, then

$$
\operatorname{dim}\left(G_{0}^{\prime}\right) \geq \min \{(n / 2)(n-1)-n+3,(n / 2)(n-1)\}
$$

We see that we can also speak about $\delta(H)$ for subspaces $H$ of $\bar{G}$.

With this definition of $\delta$ we can define a geometry on $\mathcal{S}$-structures $M$ following the approach of E. Hrushovski [12] for relational structures. We use the presentation of J.B. Goode and B. Poizat in [10]. Then we have the same geometry on $\bar{G}$ for the corresponding $G$ in $\mathcal{G}$.

Definition. For $(M, N(M))$ in $\mathcal{S}$ and $H \leq M, H$ is selfsufficient in $M$ if for all finite subspaces $K$ with $H \leq K \leq M$ we have $\delta(H) \leq \delta(K)$.

If $(\Sigma 3)^{0}$ is true for $M$, then every finite subspace of $M$ is contained in a finite selfsufficient subspace of $M$.

Lemma 3.3. For subspaces $H$ and $K$ of $M, N(H \cap K)=N(H) \cap N(K)$.

Proof. This holds because $\wedge^{2} H \cap \wedge^{2} K=\wedge^{2}(H \cap K)$.

Lemma 3.4. Let $H$ and $K$ be finite subspaces of $M$ Then

$$
\delta(H+K) \leq \delta(H)+\delta(K)-\delta(H \cap K) .
$$

Proof. $\operatorname{dim}(H+K)=\operatorname{dim}(H)+\operatorname{dim}(K)-\operatorname{dim}(H \cap K)$, and $\operatorname{dim}(N(H)+N(K))=$ $\operatorname{dim}(N(H))+\operatorname{dim}(N(K))-\operatorname{dim}(N(H \cap K))$, using Lemma 3.3. As $N(H)+N(K) \leq$ $N(H+K)$, our claim follows from the definition of $\delta$.

To define a pregeometry on $M$ (on $\bar{G}$ respectively) we only need $(\Sigma 3)^{0}$, Lemma 3.4 , and $\delta(\emptyset)=0$ (see [12]). This definition is described in the following.

Corollary 3.5. Let $H$ and $K$ be finite subspaces of $M$.

1. If $\delta(H \cap K)>\delta(H)$, then $\delta(H+K)<\delta(K)$.

2. If $\delta(H \cap K) \geq \delta(H)$, then $\delta(H+K) \leq \delta(K)$.

Lemma 3.6. If $H$ and $K$ are selfsufficient subspaces of $M$, then $A=H \cap K$ is selfsufficient in $M$.

Proof. Otherwise consider a counterexample $A=H \cap K \neq\langle 0\rangle$ such that $\delta(K) \leq$ $\delta(H)$ and $\delta(K)$ is minimal. If $\delta(A)>\delta(K)$ then, by Corollary 3.5, $\delta(K+H)<\delta(H)$. This contradicts the selfsufficiency of $H$. Therefore we can assume that $\delta(A) \leq$ $\delta(K)$. There exists $B \supseteq A$ such that $B$ is selfsufficient and $\delta(B)<\delta(A) \leq \delta(K)$. Since $\delta(B)<\delta(K)$ and $\delta(K)$ was chosen minimal for a counterexample, it follows that $B \cap K$ is selfsufficient. Note that $B \cap K \supseteq A \neq\langle 0\rangle$. We obtain a new counterexample:

$$
A=(B \cap K) \cap H \text {, where } B \cap K \text { and } H \text { are selfsufficient. }
$$

Then $\delta(B \cap K) \leq \delta(B)<\delta(K)$, contradicting the minimality of $\delta(K)$.

Let us assume for the rest of this section that $M$ satisfies $(\Sigma 3)^{0}$. Hence every finite subspace $A$ of $M$ is contained in a finite selfsufficient subspace $B$ of $M$. Therefore and because of Lemma 3.6 we can define:

Definition. Let $A$ be a finite subspace of $M$.

1. The selfsufficient closure $C S S(A)$ of $A$ is the intersection of all finite selfsufficient subspaces $B$ with $A \leq B$. 
2. The geometrical dimension $d(A)$ of $A$ is $\delta(C S S(A))$.

3. For $a_{1}, \ldots, a_{n}, a$ in $M$ let us define $a \in \operatorname{cl}\left(\left\{a_{1}, \ldots, a_{n}\right\}\right)$ if and only if

$$
d\left(\left\langle\left\{a_{1}, \ldots, a_{n}, a\right\}\right\rangle\right)=d\left(\left\langle\left\{a_{1}, \ldots, a_{n}\right\}\right\rangle\right) .
$$

By the definition, for all finite subspaces $A$ and $B$ of $M$ with $A \subseteq B$ we have $\delta(C S S(A)) \leq \delta(B)$, especially $\delta(C S S(A)) \leq \delta(A)$. We use the same notions in $\bar{G}$ for $G \in \mathcal{G}$. If $A$ is a finite subspace of $\bar{G}$, then in $G^{e q} C S S(A)$ is part of the algebraic closure of $A$. If $m=\operatorname{dim}(C S S(A))$ and $n=\delta(C S S(A))$, then $C S S(A)$ is the subspace $X$ of $\bar{G}$ that contains $A$, has linear dimension $m$, and has $\delta(X)=n$. Using this we can write a formula that says " $x \in X$ ". This formula uses elements of $G$ that represent $A$ modulo $Z(G)$ as parameters.

Lemma 3.7. Let $A$ and $B$ be subspaces of $M$ with $A \subseteq B$. Then $d(A) \leq d(B)$.

Proof. $A \subseteq B \subseteq C S S(B)$ implies $C S S(A) \subseteq C S S(B)$.

Lemma 3.8. If $d(A+\langle a\rangle)>d(A)$ then $d(A+\langle a\rangle)=d(A)+1$.

Proof. W.l.o.g. $C S S(A)=A$. By definition $d(A+\langle a\rangle)=\delta(C S S(A+\langle a\rangle)) \leq$ $\delta(A+\langle a\rangle)$. It is sufficient to show that $\delta(A+\langle a\rangle) \leq \delta(A)+1$. This follows from Lemma 3.4: $\delta(A+\langle a\rangle) \leq \delta(A)+\delta(\langle a\rangle)=\delta(A)+1$.

Theorem 3.9. cl defines a pregeometry on $M$.

Proof. (1) $\left\{a_{1}, \ldots, a_{n}\right\} \subseteq \operatorname{cl}\left(a_{1}, \ldots, a_{n}\right)$ is true by the definition. (2) For finite subsets $A \subseteq B$ we obtain $\operatorname{cl}(A) \subseteq \operatorname{cl}(B)$.

Proof of (2). W.l.o.g. $A$ and $B$ are subspaces. Assume that $a \in \operatorname{cl}(A)$. Then $d(A+\langle a\rangle)=d(A)$. Choose a selfsufficient $K$ with $A \cup\{a\} \subseteq K$ and $\delta(K)=$ $d(K)=d(A+\langle a\rangle)=d(A)$. Choose $B \subseteq H$ such that $H$ is selfsufficient and $\delta(H)=d(B)$. By Lemma $3.7 d(A) \leq d(B)$. Therefore $\delta(K)=d(A) \leq d(B)=$ $\delta(H)$. We have $A \subseteq K \cap H$. By Lemma 3.6 $K \cap H$ is selfsufficient. It follows that $d(A) \leq d(K \cap H) \leq d(K) \leq d(A)$. That means $\delta(K \cap H)=\delta(K)$. By Corollary $3.5 \delta(K+H) \leq \delta(H)=d(B)$. Therefore $d(B) \leq d(K+H) \leq \delta(K+H) \leq d(B)$. Since $B \cup\{a\} \subseteq K+H$, we get $d(B+\langle a\rangle) \leq d(K+H)=d(\bar{B})$, as desired.

(3) $\operatorname{cl}(\operatorname{cl}(A))=\operatorname{cl}(A)$ for finite $A$.

Proof of (3). By (1) $A \subseteq \operatorname{cl}(A)$, and by $(2) \operatorname{cl}(A) \subseteq \operatorname{cl}(\operatorname{cl}(A))$. To prove the other direction assume $a \in \operatorname{cl}(\operatorname{cl}(A))$. Choose $a_{1}, \ldots, a_{n} \in \operatorname{cl}(A) \backslash A$ such that $a \in$ $\operatorname{cl}\left(\left\{a_{1}, \ldots, a_{n}\right\} \cup A\right)$. Then $d(A)=d\left(A+\left\langle a_{n}\right\rangle\right)$. By $(2) a_{1}, \ldots, a_{n-1} \in \operatorname{cl}\left(A \cup\left\{a_{n}\right\}\right)$. If we iterate the argument we get $d(A)=d\left(A+\left\langle\left\{a_{1}, \ldots, a_{n}\right\}\right\rangle\right)$. Using Lemma 3.7 twice we get the desired result:

$$
\begin{aligned}
d(A+\langle a\rangle) & \leq d\left(A+\left\langle\left\{a_{1}, \ldots, a_{n}, a\right\}\right\rangle\right) \\
& =d\left(A+\left\langle\left\{a_{1}, \ldots, a_{n}\right\}\right\rangle\right)=d(A) \leq d(A+\langle a\rangle) .
\end{aligned}
$$

(4) The Steinitz Exchange Lemma. For finite $A \subseteq M, a \in \operatorname{cl}(A \cup\{b\}) \backslash \operatorname{cl}(A)$ implies $b \in \operatorname{cl}(A \cup\{a\})$.

Proof of (4). By the assumption and Lemma 3.7 we know that $d(A)<d(A+\langle a\rangle) \leq$ $d(A+\langle\{a, b\}\rangle)=d(A+\langle b\rangle)$. Hence by Lemma $3.8 d(\langle A \cup\{a, b\}\rangle)=d(A+\langle b\rangle)=$ $d(A)+1$. By assumption $d(A+\langle a\rangle)>d(A)$ and by Lemma $3.8 d(A+\langle a\rangle)=d(A)+1$. Both equations together imply $d(\langle A \cup\{a, b\}\rangle)=d(A+\langle a\rangle)$, as desired. 
Definition. Let $A$ be a finite subspace of $M$. We say that $A$ is $n$-selfsufficient in $M$ if $\delta(A) \leq \delta(B)$ for every finite $B$ with $A \subseteq B$ and $\operatorname{dim}(B / A)=n$.

Note that a subspace $A$ is selfsufficient in $M$ if and only if it is $n$-selfsufficient in $M$ for all $n$. There are formulas $\varphi_{n, m}\left(x_{1}, \ldots, x_{m}\right)$ which express the property that $x_{1}, \ldots, x_{m}$ generate an $n$-selfsufficient subspace.

Lemma 3.10. If $A$ is selfsufficient in $B$ and $B$ is selfsufficient in $M$, then $A$ is selfsufficient in $M$. If $A$ is $n$-selfsufficient in $B$ and $B$ is selfsufficient in $M$ then $A$ is $n$-selfsufficient in $M$.

Proof. For every $K \supseteq A$ we have to show that $\delta(A) \leq \delta(K)$. This is clear for $K \subseteq B$ and for $B \subseteq K$. Assume neither $K \subseteq B$ nor $B \subseteq K$. Then $\delta(A) \leq \delta(B \cap K)$ and $\delta(A) \leq \delta(B)$. If $\delta(K)<\delta(A)$ then $\delta(K)<\delta(B \cap K)$. By Corollary 3.5 $\delta(B+K)<\delta(B)$. Since $B$ is selfsufficient in $M$, this is a contradiction. The same proof works for $A n$-selfsufficient in $B$.

Lemma 3.11. Let $A$ be a finite subspace of $B$.

1. If $A$ is selfsufficient in $B$, then for every $K \subseteq B$ we obtain that $A \cap K$ is selfsufficient in $B \cap K=K$.

2. If $A$ is $n$-selfsufficient in $B$, then for every $K \subseteq B$ we obtain that $A \cap K$ is $n$-selfsufficient in $B \cap K=K$.

Proof. To prove (1) we have to show that for every $C$ with $A \cap K \subseteq C \subseteq K$ we have $\delta(A \cap K) \leq \delta(C)$. Note that $A \cap K=A \cap C$. Now $\delta(C)<\delta(A \cap C)$ would imply $\delta(A+C)<\delta(A)$ by Corollary 3.5. This is a contradiction to the selfsufficiency of $A$ in $B$.

(2) follows in the same way. We have to consider $C$ with $\operatorname{dim}(C / A \cap C) \leq n$ only. Note that $\operatorname{dim}((A+C) / A)=\operatorname{dim}(C / A \cap C)$.

\section{The First Amalgamation Lemma}

Let $A, B$, and $C$ be structures in $\mathcal{S}$, where $A$ is a common substructure of $B$ and $C$. We define a structure $D=B \star_{A} C$, that we call the free amalgam of $B$ and $C$ over $A$ : Let $D$ be the vector space that is the free amalgam $B \oplus_{A} C$ of the vector spaces $B$ and $C$ over $A$ in the category of vector spaces over the field with $p$ elements. It remains to define the subspace $N(D)$ of $\wedge^{2} D . \wedge^{2} B, \wedge^{2} C$ and $\wedge^{2} A$ are subspaces of $\wedge^{2} D$ with $\wedge^{2} B \cap \wedge^{2} C=\wedge^{2} A$. Therefore $N(B)$ and $N(C)$ are subspaces of $\wedge^{2} D$ with $N(B) \cap N(C)=N(A)$ (Lemma 3.3). We define $N(D)$ to be the subspace of $\wedge^{2} D$ generated by $N(B) \cup N(C)$. In particular we have $\delta(D)=\delta(B)+\delta(C)-\delta(A)$. Under certain conditions we will show $(\Sigma 3)$ for $D$ provided that $A, B$, and $C$ fulfill $(\Sigma 3)$.

Let $(M, N(M))$ be a structure in $\mathcal{S}$ with $(\Sigma 3)^{0}$ and let $H \subseteq K$ be subspaces of $M$. We say that $K$ is a minimal extension of $H$ if $\delta(H)=\delta(\bar{K})$ and $\delta(L)>\delta(H)$ for every $L$ with $H \subseteq L \subseteq K$ and $H \neq L \neq K$. We also use this notion for the induced $\mathcal{S}$-substructures. $K_{1}$ is a realization of the minimal extension $K$ of $H$, if there is a vector space isomorphism of $K$ onto $K_{1}$ that is the identity on $H$ and that induces an isomorphism of the corresponding structures in $\mathcal{S}$.

Theorem 4.1 (First Amalgamation Theorem). Assume that A, B, $C$ are finite structures in $\mathcal{S}$ that fulfill $(\Sigma 3)$, where $A$ is a common substructure of $B$ and $C$. Suppose that $A$ is selfsufficient in $B$ and $n$-selfsufficient in $C$, where $2+\operatorname{dim}(B / A) \leq$ $n$. Then $D=B \star_{A} C$ fulfills $(\Sigma 3)^{0}, C$ is selfsufficient in $D$ and $B$ is $n$-selfsufficient 
in $D$. If $B$ is a minimal extension of $A$ that is not realized in $C$, or if $\operatorname{dim}(B)=$ $\operatorname{dim}(A)+1$ and $\delta(B)=\delta(A)+1$, then $D$ fulfills $(\Sigma 3)$.

Let us introduce the following notation. If $M$ is an $\mathcal{S}$-structure and $X$ is an ordered basis of the vector space $M$, then we call the elements $a \wedge b$ of $\wedge^{2} M$ with $a<b$ the basic commutators. They form a basis of $\wedge^{2} M$. If we speak about the basic commutators of $\wedge^{2} M$ with respect to the basis $X$, then we assume that some ordering of $X$ is given. If $X$ is of the form $X_{1} \ldots X_{n}$, then we assume that the ordering of $X$ has the property $x<y$ for $x \in X_{i}$ and $y \in X_{j}$ with $i<j$.

First we prove some helpful lemmas. If we want to show $(\Sigma 3)$, we have to consider any $K \subseteq D$. Then $(K \cap B) \cap(K \cap C)=K \cap(B \cap C)=K \cap A$. Let $Y^{a}$ be a basis for the vector space $K \cap A, Y^{a} Y^{b}$ be a basis for $K \cap B$, and $Y^{a} Y^{c}$ be a basis of $K \cap C$. Then $Y^{b} Y^{c}$ is linearly independent over $A$. If $\operatorname{dim}(K)=$ $\operatorname{dim}(K \cap B)+\operatorname{dim}(K \cap C)-\operatorname{dim}(K \cap A)$ then $Y^{a} Y^{b} Y^{c}$ is a basis for $K$. By induction on $\operatorname{dim}(K)-(\operatorname{dim}(K \cap B)+\operatorname{dim}(K \cap C)-\operatorname{dim}(K \cap A))$ we show:

Lemma 4.2. Let $K$ be a finite subspace of $D$. Assume that $d_{1}, \ldots, d_{m}$ are linearly independent over $(K \cap B)+(K \cap C)$ such that

$$
\left\langle(K \cap B) \cup(K \cap C) \cup\left\{d_{1}, \ldots, d_{m}\right\}\right\rangle=K .
$$

Then for every $U=\left\{u_{1}, \ldots, u_{m}\right\} \subseteq B$ and $V=\left\{v_{1}, \ldots, v_{m}\right\} \subseteq C$ with $u_{i}=d_{i}$ modulo $C$ and $d_{i}=u_{i}+v_{i}$ for $1 \leq i \leq m$ we obtain that $U V$ is linearly independent over $(K \cap B)+(K \cap C)+A$.

Proof. $m=0$ is the base of the induction. Otherwise choose

$$
H=\left\langle(K \cap B) \cup(K \cap C) \cup\left\{d_{1}, \ldots, d_{m-1}\right\}\right\rangle .
$$

Then $\operatorname{dim}(H)-(\operatorname{dim}(K \cap B)+\operatorname{dim}(K \cap C)-\operatorname{dim}(K \cap A))=m-1$. We apply the induction hypothesis and obtain that $Y^{b} Y^{c}\left\{u_{1}, \ldots, u_{m-1}\right\}\left\{v_{1}, \ldots, v_{m-1}\right\}$ is linearly independent over $A$. If the assertion were not true, we could infer

$$
\sum_{1 \leq i \leq m} r_{i} u_{i}+\sum_{1 \leq i \leq m} s_{i} v_{i}=0 \text { modulo }\left\langle Y^{b} Y^{c} A\right\rangle
$$

where $r_{m} \neq 0$ or $s_{m} \neq 0$. Assume w.l.o.g. that $s_{m} \neq 0$. Then there is some $z$ in $\left\langle Y^{c}\right\rangle$ such that

$$
\sum_{1 \leq i \leq m} s_{i} d_{i}+z=\sum_{1 \leq i \leq m}\left(s_{i}-r_{i}\right) u_{i} \text { modulo }\left\langle Y^{b} A\right\rangle .
$$

It follows that there is some $h \in H$ such that $s_{m} d_{m}+h \in K \cap B$. Hence $d_{m} \in H$, a contradiction.

A basis $Y^{a} Y^{b} Y^{c}\left\{u_{1}+v_{1}, \ldots, u_{m}+v_{m}\right\}$ of a subspace $K$ of $D$ as described in Lemma 4.2 is called a suitable basis. A suitable basis $Z^{a} Z^{b} Z^{c}$ of $D$ is consistent with the suitable basis $Y^{a} Y^{b} Y^{c}\left\{u_{1}+v_{1}, \ldots, u_{m}+v_{m}\right\}$ of $K$, if $Y^{a} \subseteq Z^{a}, Y^{b} \subseteq Z^{b}$, $Y^{c} \subseteq Z^{c}$, and the roles of $\left\{u_{1}, \ldots, u_{m}\right\}$ and $\left\{v_{1}, \ldots, v_{m}\right\}$ can be played by elements of $Z^{b} \backslash Y^{b}$ and $Z^{c} \backslash Y^{c}$ respectively. The description of $K$ above gives us a description of $N(K)=N(D) \cap \wedge^{2} K$.

Lemma 4.3. Let $Y^{a} Y^{b} Y^{c} U V$ be a part of a basis of $D$ chosen according to $K$ as above in Lemma 4.2. Assume that there is given an order of this basis with $Y^{a}<Y^{b}<Y^{c}<U<V$. Then a linear combination $\Phi$ of basic commutators over $Y^{a} Y^{b} Y^{c} U V$ is in $N(D)$ if and only if $\Phi=\Phi_{B}+\Phi_{C}+\Phi_{U}+\Phi_{V}$, where: 
$\Phi_{B}$ is a linear combination of basic commutators $a \wedge b$ with $a, b \in Y^{a} Y^{b}$ and $a<b$,

$\Phi_{C}$ is a linear combination of basic commutators $a \wedge b$ with $a, b \in Y^{a} Y^{c}$ and $a<b$,

$\Phi_{U}$ is a linear combination of basic commutators $a \wedge u_{j}$ with $a \in Y^{a}$ and $u_{j} \in U$, $\Phi_{V}$ is obtained from $\Phi_{U}$ by replacing $u_{j}$ by $v_{j}$,

and there exists $J \in \wedge^{2} A$ such that $\Phi_{B}+\Phi_{U}+J \in N(B)$ and $\Phi_{C}+\Phi_{V}-J \in$ $N(C)$.

Proof. $\wedge^{2} K$ has a basis $\left\{a \wedge b: a<b, a, b \in Y^{a} Y^{b} Y^{c}\left\{u_{i}+v_{i}: 1 \leq i \leq m\right\}\right\}$. Let $\Phi$ be a linear combination of such basic commutators that is in $N(D)$. With respect to any basis of $D$, that extends $Y^{a} Y^{b} Y^{c} U V$, we have $\Phi=\Phi_{B}+\Phi_{C}+\sum_{i} \alpha_{i}\left(a_{i} \wedge u_{i}\right)+$ $\sum_{i} \alpha_{i}\left(a_{i} \wedge v_{i}\right)$, where $\Phi_{B} \in \wedge^{2}\left\langle Y^{a} Y^{b}\right\rangle, \Phi_{C} \in \wedge^{2}\left\langle Y^{a} Y^{c}\right\rangle$, and $a_{i} \in Y^{a} Y^{b} Y^{c}\left\{u_{l}+v_{l}\right.$ : $1 \leq l \leq i\}$, because $b \wedge c$ with $b \in Y^{b}$ and $c \in Y^{c}$ cannot occur in $\Phi \in N(B)+$ $N(C)$. By the same argument $r b, r c, r\left(u_{i}+v_{i}\right)$ with $b \in Y^{b}$ and $c \in Y^{c}$ cannot occur in the presentation with respect to the basis above of any $a_{j}$. Therefore $a_{i} \in\left\langle Y^{a}\right\rangle$. We define $\Phi_{U}=\sum_{i} \alpha_{i}\left(a_{i} \wedge u_{i}\right)$ and $\Phi_{V}=\sum_{i} \alpha_{i}\left(a_{i} \wedge v_{i}\right)$. We get $\Phi=\Phi_{B}+\Phi_{U}+\Phi_{C}+\Phi_{V}=\Psi_{B}+\Psi_{C}$, where $\Psi_{B} \in N(B)$ and $\Psi_{C} \in N(C)$. Let $J$ be $\Psi_{B}-\left(\Phi_{B}+\Phi_{U}\right)=-\Psi_{C}+\left(\Phi_{C}+\Phi_{V}\right)$. Then $J \in \wedge^{2} B \cap \wedge^{2} C=\wedge^{2} A$. The assertion follows.

Lemma 4.4. Assume that $A, B$, and $C$ are finite structures in $\mathcal{S}$ with $(\Sigma 3)$, such that $A$ is a common substructure of $B$ and $C$. Let $D$ be $B \star_{A} C$. Suppose that $A$ is selfsufficient in $B$, and $A$ is $(\operatorname{dim}(B / A)+n)$-selfsufficient in $C$, and $B$ is a minimal extension of $A$, that is not realized in $C$. Assume that $U=\left\{u_{1}, \ldots, u_{m}\right\} \subseteq B$ and $V=\left\{v_{1}, \ldots, v_{m}\right\} \subseteq C$, such that $U V$ is linearly independent over $A$. Let $X=\left\{a_{1}, \ldots, a_{k}\right\}$ be a part of a basis of $A$. For $1 \leq i \leq h$ let

$$
\Phi_{i}=\sum_{\substack{1 \leq s \leq k \\ 1 \leq t \leq m}} r_{s t}^{i}\left(a_{s} \wedge u_{t}+v_{t}\right)
$$

be relations in $N\left(\left\langle X\left\{u_{1}+v_{1}, \ldots, u_{m}+v_{m}\right\}\right\rangle\right)$ that are linearly independent over $N(\langle X\rangle)$. Then $h<m$.

Proof. Assume that $h \geq m$. By Lemma 4.3 there exists $J_{i} \in \wedge^{2} A$ such that

$$
\begin{aligned}
\Psi_{i} & =\sum_{\substack{1 \leq s \leq k \\
1 \leq t \leq m}} r_{s t}^{i}\left(a_{s} \wedge u_{t}\right)+J_{i} \in N(B), \\
\Delta_{i} & =\sum_{\substack{1 \leq s \leq k \\
1 \leq t \leq m}} r_{s t}^{i}\left(a_{s} \wedge-v_{t}\right)+J_{i} \in N(C) .
\end{aligned}
$$

Then $\Phi_{i}=\Psi_{i}-\Delta_{i}$. We can write down $J_{i}$ as a linear combination of basic commutators over some basis of $A$ that extends $X$. Since the $\Phi_{i}$ 's are linearly independent over $\wedge^{2}\langle X\rangle$, every non-trivial linear combination of them contains $a_{s} \wedge u_{t}$ and $a_{s} \wedge v_{t}$ for some $s$ and $t$. Therefore every nontrivial linear combination of the $\Psi_{i}$ 's and every nontrivial linear combination of the $\Delta_{i}$ 's contains a basic commutator $a_{s} \wedge u_{t}$ and $a_{s} \wedge v_{t}$ respectively. Hence the $\Psi_{i}$ 's and the $\Delta_{i}$ 's respectively are linearly independent over $\wedge^{2} A$. They freely generate $N(\langle U A\rangle)$ over $N(A)$ and $N(\langle V A\rangle)$ over $N(A)$ respectively, since $A$ is $\operatorname{dim}(B / A)$-selfsufficient in $B$ and $C$, $m \leq \operatorname{dim}(B / A)$, and $h \geq m$. It follows that $h=m$. Therefore the vector space 
isomorphism $f$, defined by $f(a)=a$ for $a \in A$ and $f\left(u_{j}\right)=-v_{j}$ of $\langle A U\rangle$ onto $\langle A V\rangle$ induces an isomorphism of the corresponding structures in $\mathcal{S}$. Since $B$ is a minimal extension of $A$ and $\delta(\langle A U\rangle)=\delta(A)$ (in $B$ ), we obtain $\langle A U\rangle=B$. Now $\langle A V\rangle$ is a realization of the minimal extension $B$ of $A$ in $C$. This contradicts the assumption of the lemma.

Lemma 4.5. Assume that $M$ is a structure in $\mathcal{S}$ sucht that $\delta(K)=\operatorname{dim}(K)$ for $K \subseteq M$ with $\operatorname{dim}(K) \leq 2$. Then $\delta(K)=\operatorname{dim}(K)$ for $K \leq M$ with $\operatorname{dim}(K) \leq 3$.

Proof. Choose $K \subseteq M$ with $\operatorname{dim}(K)=3$. Assume that $K$ has the basis $\{a, b, c\}$. If $\delta(K)<3$ then there is a relation $r_{1}(a \wedge b)+r_{2}(a \wedge c)+r_{3}(b \wedge c)$ in $N(K)$. Assume w.l.o.g. that $r_{1} \neq 0$. If $r_{3}=0$, then $r_{2} \neq 0$ by assumption. But then $a \wedge\left(r_{1} b+r_{2} c\right) \in N(K)$, a contradiction. We obtain a similar contradiction if $r_{2}=0$. Therefore we can assume w.l.o.g. that $r_{1}=1, r_{2} \neq 0$, and $r_{3} \neq 0$. Hence $a \wedge\left(b+r_{2} c\right)+r_{3}(b \wedge c) \in N(K)$. Choose $s$ such that $s r_{2}=r_{3}$ modulo $p$. Then $a \wedge\left(b+r_{2} c\right)+s b \wedge\left(b+r_{2} c\right) \in N(K)$ and $(a+s b) \wedge\left(b+r_{2} c\right) \in N(K)$, a contradiction.

Proof of Theorem 4.1. We show $(\Sigma 3)^{0}$ for $D$ and $(\Sigma 3)$ under the further assumptions. Let $K \subseteq D$. $Y^{a}, Y^{b}, Y^{c}, U$ and $V$ are chosen as above. If $\operatorname{dim}(K) \leq 2$ then by Lemma 4.3 either $N(K)=\langle 0\rangle$ or $K=\langle\{a, u+v\}\rangle$, where $a \in A, u \in B, v \in C$, $\{u, v\}$ is linearly independent over $A$, and $N(K)$ is generated by $a \wedge(u+v)$. It follows that $\delta(K) \geq 1$. This implies $(\Sigma 3)^{0}$ for $K$ with $\operatorname{dim}(K) \leq 2$. Assume that $\operatorname{dim}(K)=2$. If $\operatorname{dim}(B)=\operatorname{dim}(A)+1$ and $\delta(B)=\delta(A)+1$ then $\operatorname{dim}(N(K))=0$, since a relation $a \wedge u+J \in N(B)$ is not possible. If $B$ is a minimal extension of $A$ that is not realized in $C$, then $\operatorname{dim}(N(K))=0$ follows from Lemma 4.4. Hence the assertion is proved for all $K \subseteq M$ with $\operatorname{dim}(K) \leq 2$.

Now we can suppose that $\operatorname{dim}(K) \geq 3$. We choose a basis for $K$ as above. We say that $K$ is moderate if $U=V=\emptyset$. First we show $(\Sigma 3)$ for moderate $K$. Using Lemma 4.3 we prove for moderate $K$ :

(1) $\operatorname{dim}(N(K)) \leq \operatorname{dim}(N(K \cap C)+\operatorname{dim}(N((K \cap B)+A))-\operatorname{dim}(N(A))$.

Proof of (1). $N(K)$ is a subspace of $\wedge^{2} K=\wedge^{2}((K \cap B)+(K \cap C))$. We construct a basis of $N(K)$. First we choose $\Phi_{1}, \ldots, \Phi_{s}$ as a basis of $N(K \cap C)$. Let $\Delta_{1}, \ldots, \Delta_{r}$ be linear combinations of basic commutators in $N(K)$ such that their images in $N(K) / N(K \cap C)$ are a basis of this vector space. By Lemma $4.3 \Delta_{i}=\Delta_{i}^{B}+J_{i}+$ $\Delta_{i}^{C}-J_{i}$ where $\Delta_{i}^{B} \in \wedge^{2}(K \cap B), \Delta_{i}^{C} \in \wedge^{2}(K \cap B), J_{i} \in \wedge^{2} A, \Delta_{i}^{B}+J_{i} \in N(B)$, and $\Delta_{i}^{C}-J_{i} \in N(C) . \Delta_{i}^{B}$ is a linear combination of basic commutators over $Y^{a} Y^{b}$. Then every non-trivial linear combination $\Delta$ of the $\Delta_{i}^{B}$ contains a basic commutator $w \wedge y$ with $y \in Y^{b}$, since otherwise the corresponding linear combination of the $\Delta_{i}$ 's would be in $N(K \cap C)$. Hence the $\Delta_{i}^{B}+J_{i} \in N((K \cap B)+A)$ are linearly independent over $\wedge^{2} A$ and therefore over $N(A)$.

Note that $\operatorname{dim}(K)=\operatorname{dim}(K \cap C)+\operatorname{dim}((K \cap B)+A)-\operatorname{dim}(A)$. Therefore (1) implies

(2) $\delta(K) \geq \delta(K \cap C)+\delta((K \cap B)+A)-\delta(A)$.

Since $A$ is selfsufficient in $B$ we obtain $\delta((K \cap B)+A) \geq \delta(A)$, and therefore

(3) $\delta(K) \geq \delta(K \cap C)$.

$(\Sigma 3)^{0}$ follows for moderate $K$ and $(\Sigma 3)$ for moderate $K$ with $\operatorname{dim}(K \cap C) \geq 3$. 
If $\operatorname{dim}(B)=\operatorname{dim}(A)+1$ and $\delta(B)=\delta(A)+1$, then either $K \subseteq C$ or $\delta(K)=$ $\delta(K \cap C)+1$ by $(2)$. The assertion follows from $(\Sigma 3)$ for $C$. Now we can assume that $B$ is a minimal extension of $A$, that is not realized in $C$. Similarly as in (2) we obtain

(4) $\delta(K) \geq \delta(K \cap B)+\delta((K \cap C)+A)-\delta(A)$.

It suffices to consider the cases where $\left|Y^{c}\right| \leq 2$. Since $\operatorname{dim}((K \cap C)+A / A)=$ $\left|Y^{c}\right| \leq 2$, the 2-selfsufficiency of $A$ in $C$ implies

(5) $\delta(K) \geq \delta(K \cap B)$, if $\left|Y^{c}\right| \leq 2$.

The assertion follows if $\operatorname{dim}(K \cap B) \geq 3$. Hence we can suppose that $\operatorname{dim}(K \cap C)<3$ and $\operatorname{dim}(K \cap B)<3$. At the beginning $(\Sigma 3)$ was shown for all $K$ with $\operatorname{dim}(K) \leq 2$. By Lemma 4.5 it is true for $K$ with $\operatorname{dim}(K) \leq 3$. We can assume that $\operatorname{dim}(K)>3$. The only possibility to fulfill all these conditions is $\left|Y^{a}\right|=0,\left|Y^{b}\right|=\left|Y^{c}\right|=2$. In this case we show $\operatorname{dim}(N(K)) \leq 1$. Let $Y^{b}$ be $\left\{y_{1}, y_{2}\right\}$ and $Y^{c}$ be $\left\{z_{1}, z_{2}\right\}$. By $(\Sigma 3)$ for $B$ and $C, y_{1} \wedge y_{2}$ and $z_{1} \wedge z_{2}$ are not in $N(K)$. Therefore by Lemma 4.3 every element in $N(K)$ has the form $r_{1}\left(y_{1} \wedge y_{2}\right)+r_{2}\left(z_{1} \wedge z_{2}\right)$ with $r_{1} \neq 0$ and $r_{2} \neq 0$. There are not two linearly independent elements of this form in $N(K)$, since their existence would imply $y_{1} \wedge y_{2} \in N(K)$. It follows that $\operatorname{dim}(N(K)) \leq 1$, as desired. $(\Sigma 3)$ is shown for moderate $K$.

Now we assume that $K$ is not moderate. Remember that by assumption $\operatorname{dim}(K)$ $\geq 3$. Let $K^{-}$be $(K \cap B)+(K \cap C)=\left\langle Y^{a} Y^{b} Y^{c}\right\rangle$. $K^{-}$is moderate. By definition $N\left(K^{-}\right) \subseteq N(K)$. We use a similar proof as in the moderate case. First we show

(6) $\operatorname{dim}(N(K)) \leq \operatorname{dim}(N(K \cap C))+\operatorname{dim}\left(N\left(\left\langle Y^{b} U A\right\rangle\right)\right)-\operatorname{dim}(N(A))$.

Proof of (6). Let $\Phi_{1}, \ldots, \Phi_{s}$ be a basis of $N(K \cap C)$. Let $\Delta_{1}, \ldots, \Delta_{r}$ in $N\left(K^{-}\right)$ be a basis of $N\left(K^{-}\right) / N(K \cap C)$. In the proof of (1) it is shown that $\Delta_{1}^{+}=$ $\Delta_{1}^{B}+J_{1}, \ldots, \Delta_{r}^{+}=\Delta_{r}^{+}+J_{r} \in N\left(\left\langle Y^{b} A\right\rangle\right)$ are linearly independent over $\wedge^{2} A$. Now we choose $\Psi_{1}, \ldots, \Psi_{t}$ in $N(K)$ such that their images in $N(K) / N\left(K^{-}\right)$are a basis of this vector space. Then $\left\{\Phi_{1}, \ldots, \Phi_{s}, \Delta_{1}, \ldots, \Delta_{r}, \Psi_{1}, \ldots, \Psi_{t}\right\}$ is a basis of $N(K)$. According to Lemma 4.3 let $\Psi_{i}=\Psi_{i}^{B}+\Psi_{i}^{C}+\Psi_{i}^{U}+\Psi_{i}^{V}$ and $I_{i} \in \wedge^{2} A$ with $\Psi_{i}^{B}+\Psi_{i}^{U}+I_{i} \in N(B)$ and $\Psi_{i}^{C}+\Psi_{i}^{V}-I_{i} \in N(C)$. Then every non-trivial linear combination of the $\Psi_{i}$ 's contains some $a \wedge u_{j}$ with $a \in Y^{a}$ and $u_{j} \in U$. Therefore the $\Psi_{i}^{+}=\Psi_{i}^{B}+\Psi_{i}^{U}+I_{i} \in N(B)$ are linearly independent over $\left\langle\wedge^{2} A \cup\left\{\Delta_{1}^{+}, \ldots, \Delta_{r}^{+}\right\}\right\rangle$. Claim (6) follows, since $\Delta_{1}^{+}, \ldots, \Delta_{r}^{+}, \Psi_{1}^{+}, \ldots, \Psi_{t}^{+}$are elements of $N\left(\left\langle Y^{b} U A\right\rangle\right)$ that are linearly independent over $\wedge^{2} A$.

As above it follows that

(7) $\delta(K) \geq \delta(K \cap C)+\delta\left(\left\langle Y^{b} U A\right\rangle\right)-\delta(A)$.

By the selfsufficiency of $A$ in $B$ we obtain that $\delta\left(\left\langle Y^{b} U A\right\rangle\right)-\delta(A) \geq 0$ and therefore

(8) $\delta(K) \geq \delta(K \cap C)$.

$(\Sigma 3)^{0}$ follows for all $K$, as does $(\Sigma 3)$ for all $K$ with $\operatorname{dim}(K \cap C) \geq 3$. Note that we have assumed that $\operatorname{dim}(K) \geq 3$ and $K$ is not moderate. If $\operatorname{dim}(B)=\operatorname{dim}(A)+1$ and $\delta(B)=\delta(A)+1$, then $\operatorname{dim}(K \cap C)=\operatorname{dim}(K)-1,\left|Y^{b}\right|=0,|U|=1$, and $\delta\left(\left\langle Y^{b} U A\right\rangle\right)-\delta(A)=1$. Therefore $(\Sigma 3)$ follows from $(7)$ in this case.

Now we assume that $B$ is a minimal extension of $A$ that is not realized in $C$. The assertion is proved for $\operatorname{dim}(K \cap C) \geq 3$. If $\operatorname{dim}(K \cap C)<3$, then we can show analogously as above that $\delta(K) \geq \delta(K \cap B)$. We use the 2-selfsufficiency of $A$ in $C$. Therefore we can assume now that $\operatorname{dim}(K \cap C)<3$ and $\operatorname{dim}(K \cap B)<3$. The following cases are possible: $\left|Y^{a}\right|=0,\left|Y^{a}\right|=1$, and $\left|Y^{a}\right|=2$. 
Case $\left|Y^{a}\right|=0$ : By Lemma $4.3 N(K)=N\left(K^{-}\right)$. Therefore $\delta(K)=\operatorname{dim}(K)-$ $\operatorname{dim}\left(K^{-}\right)+\delta\left(K^{-}\right)$. Since the assertion is already proved for the moderate $K^{-}$, it follows for $K$.

Case $\left|Y^{a}\right|=1$ : Since $\operatorname{dim}(K \cap C)<3$ and $\operatorname{dim}(K \cap B)<3,\left|Y^{b}\right|,\left|Y^{c}\right| \leq 1$. Let $\Phi$ be any non-trivial relation in $N(K)$. Note that $N\left(\left\langle Y^{a}\right\rangle\right)=0$. If $Y^{a}=\{a\}$, then by Lemma $4.3 \Phi=a \wedge(u+v)$, where $u \in\left\langle Y^{b} U\right\rangle$ and $v \in\left\langle Y^{c} V\right\rangle . u=0$ or $v=0$ is impossible by $(\Sigma 3)$ for $B$ and $C$. By Lemma $4.4 \operatorname{dim}(N(\langle\{a, u+v\}\rangle))=0$ and therefore $\operatorname{dim}(N(K))=0$. Hence $\delta(K)=\operatorname{dim}(K)$, as desired.

Case $\left|Y^{a}\right|=2$ : By assumption $\left|Y^{b}\right|=\left|Y^{c}\right|=0$. Let $Y^{a}$ be $\left\{a_{1}, a_{2}\right\},|U|=l$. We suppose that there are at least $l$ relations $\Phi_{i}$ in $N(K)$ that are linearly independent over $N\left(\left\langle Y^{a}\right\rangle\right)=\langle 0\rangle$, and show a contradiction. By Lemma 4.3:

(9) $\Phi_{i}=\sum_{\substack{s=1,2 \\ 1 \leq t \leq l}} r_{s t}^{i}\left(a_{s} \wedge\left(u_{t}+v_{t}\right)\right)+r_{0}^{i}\left(a_{1} \wedge a_{2}\right)$.

We show that it is possible to replace every $u_{t}$ by another element $u_{t}^{+}$of its coset modulo $\left\langle Y^{a}\right\rangle$, such that the representation of (9) with respect to this basis $Y^{a} U^{+} V$ has no summands $r_{0}^{i}\left(a_{1} \wedge a_{2}\right)$. Then we apply Lemma 4.4 and obtain a contradiction. It follows that $\operatorname{dim}\left(N\left(\left\langle Y^{a}\left\{u_{1}+v_{1}, \ldots, u_{l}+v_{l}\right\}\right\rangle\right)\right) \leq l-1$. Therefore $\delta(K) \geq 3$, as desired.

To get the desired basis $Y^{a} U^{+} V$ we start with $\Phi_{1}$. Since $\Phi_{1} \notin N\left(\left\langle Y^{a}\right\rangle\right)$ there are $s_{1}$ and $t_{1}$ such that $r_{s_{1} t_{1}}^{1} \neq 0$. For each $i$ with $2 \leq i \leq l$ we subtract a suitable $q_{i} \Phi_{1}$ from $\Phi_{i}$. Therefore we can assume w.l.o.g. that $r_{s_{1} t_{1}}^{i}=0$ for $2 \leq i \leq l$. Using the linear independence of $\Phi_{1}, \ldots, \Phi_{l}$ over $\wedge^{2} A$ we iterate this procedure. Therefore we can assume w.l.o.g. that there are pairwise distinct pairs $\left(s_{i}, t_{i}\right)$ such that $r_{s_{i} t_{i}}^{i} \neq 0$ and $r_{s_{i} t_{i}}^{j}=0$ for $i \neq j$. For each $t$ with $1 \leq t \leq l$ we define $u_{t}^{+}$:

(i) If there is no $i$ with $t_{i}=t$, then $u_{t}^{+}=u_{t}$.

(ii) If there is exactly one $i$ with $t_{i}=t$, then $u_{t}^{+}=u_{t}+(-1)^{3-s_{i}}\left(r_{0}^{i} / r_{s_{i} t_{i}}^{i}\right) a_{3-s_{i}}$.

(iii) If $t_{i}=t_{j}=t$ for $i \neq j$, then

$$
u_{t}^{+}=u_{t}+(-1)^{3-s_{i}}\left(r_{0}^{i} / r_{s_{i} t}^{i}\right) a_{3-s_{i}}+(-1)^{3-s_{j}}\left(r_{0}^{j} / r_{s_{j} t}^{j}\right) a_{3-s_{j}} .
$$

Note that in case (iii) $s_{i}=3-s_{j}$ and $s_{j}=3-s_{i}$. Now we write down (9) with respect to the basis $Y^{a} U^{+} V$ : Let $I$ be the set of all $t$ with $1 \leq t \leq l$ and $t \neq t_{j}$ for all $j$. Let $J_{i}$ be the set of all $t$ with $1 \leq t \leq l$ and $t=t_{j}$ for exactly one $j$, and this $j$ is not $i$.

$$
\begin{aligned}
\Phi_{i}= & \sum_{\substack{s=1,2 \\
t \in I}} r_{s t}^{i}\left(a_{s} \wedge\left(u_{t}+v_{t}\right)\right) \\
& +r_{s_{i} t_{i}}^{i}\left(a_{s_{i}} \wedge\left(u_{t_{i}}+v_{t_{i}}+(-1)^{3-s_{i}}\left(r_{0}^{i} / r_{s_{i} t_{i}}^{i}\right) a_{3-s_{i}}\right)\right) \\
& +r_{3-s_{i} t_{i}}^{i}\left(a_{3-s_{i}} \wedge\left(u_{t_{i}}+v_{t_{i}}+(-1)^{3-s_{i}}\left(r_{0}^{i} / r_{s_{i} t_{i}}^{i}\right) a_{3-s_{i}}\right)\right) \\
& +\sum_{t_{j} \in J_{i}} r_{3-s_{j} t_{j}}^{i}\left(a_{3-s_{j}} \wedge\left(u_{t}+v_{t}+(-1)^{3-s_{j}}\left(r_{0}^{j} / r_{s_{j} t_{j}}^{j}\right) a_{3-s_{j}}\right)\right) \\
= & \sum_{\substack{s=1,2 \\
1 \leq t \leq l}} r_{s t}^{i}\left(a_{s} \wedge\left(u_{t}^{+}+v_{t}\right)\right) .
\end{aligned}
$$

Note that $r_{s_{j} t_{j}}^{i}=0$ for $i \neq j$. If there is some $j \neq i$ such that $t_{j}=t_{i}$, then $r_{3-s_{i} t_{i}}^{i}=r_{s_{j} t_{j}}^{i}=0$. This finishes the proof of $(\Sigma 3)$ for the situation where $B$ is a minimal extension of $A$ that is not realized in $C$. 
Next we show that $C$ is selfsufficient in $D$. If $C \subseteq K$, then $K$ is moderate, $K \cap C=C$, and by $(3) \delta(K) \geq \delta(K \cap C)=\delta(C)$, as desired. Finally we prove that $B$ is $n$-selfsufficient in $D$. If $B \subseteq K$ and $\operatorname{dim}(K / B) \leq n$, then $K$ is moderate, $K \cap B=B$ and by $(4) \delta(K) \geq \delta(B)+\delta(K \cap C)-\delta(A)$. Since $\operatorname{dim}((K \cap C) / A)=$ $\operatorname{dim}(K / B) \leq n$, it follows that $\delta(K) \geq \delta(B)$ by the $n$-selfsufficiency of $A$ in $C$, as desired.

\section{Minimal Extensions}

Let $D$ be $B \star_{A} C$ as in Theorem 4.1, where $A, B$, and $C$ are finite structures in $\mathcal{S}$ that satisfy ( $\Sigma 3), A$ is a common substructure of $B$ and $C, A$ is selfsufficient in $B$ and $(\operatorname{dim}(B / A)+n)$-selfsufficient in $C$. Furthermore we suppose that $B$ is a minimal extension of $A$ that is not realized in $C$. By Theorem 4.1 $D$ satisfies $(\Sigma 3), C$ is selfsufficient in $D$, and $B$ is $n$-selfsufficient in $D$. We consider a minimal extension $K$ of $H$ (both finite) in $D$. As defined above, this means that $\delta(H)=\delta(K)$ but $\delta(L)>\delta(H)$ for every $L$ with $H \subseteq L \subseteq K$ and $H \neq L \neq K$. According to Lemma 4.2 we choose a suitable basis for $H$ in the following way:

Let $Y^{a}$ be a basis for $H \cap A$.

Let $Y^{b}$ be a sequence such that $Y^{a} Y^{b}$ is a basis for $H \cap B$.

Let $Y^{c}$ be a sequence such that $Y^{a} Y^{c}$ is a basis for $H \cap C$.

Let $Y^{d}$ be a basis of $H$ over $(H \cap B)+(H \cap C)$.

Then $Y^{a} Y^{b} Y^{c} Y^{d}$ is a suitable basis of $H$.

Now we start with $Y^{a} Y^{b} Y^{c} Y^{d}$, which we will extend to a suitable basis for $K$. First we choose $X^{a}$ such that $Y^{a} X^{a}$ is a basis for $K \cap A$. Let $X^{b y}$ be a basis of $K \cap B \cap(H+C)$ over $(H \cap B)+(K \cap A)$. For every $u \in X^{b y}$ we choose a $v \in C$ such that $u+v \in H . v$ exists by definition. It follows that $v \in C \cap K . v$ is uniquely determined modulo $(H \cap C)+(K \cap A)$. Let $X^{c y}$ be the set of these $v$ 's. Then $X^{c y}$ is a basis of $K \cap C \cap(H+B)$ over $(H \cap C)+(K \cap A)$. By construction we obtain $\left|X^{b y}\right|=\left|X^{c y}\right|$. For every $u \in X^{b y}$ there is exactely one $v \in X^{c y}$ such that $u+v \in H$, and for every $v \in X^{c y}$ there is exactely one $u \in X^{b y}$ with $u+v \in H$. Then we choose $X^{b x}$ and $X^{c x}$ such that $Y^{a} X^{a} Y^{b} X^{b y} X^{b x}$ is a basis for $K \cap B$ and $Y^{a} X^{a} Y^{c} X^{c y} X^{c x}$ is a basis for $K \cap C$.

Now $Y^{a} X^{a} Y^{b} X^{b y} X^{b x} Y^{c} X^{c y} X^{c x}$ is a basis for $(K \cap B)+(K \cap C)$. It can be extended to a basis of $K$. First we look for a basis of $H$ over $(K \cap B)+(K \cap C)$ and then we extend it to a basis of $K$. By Lemma 4.2 there are

$$
\begin{aligned}
Y^{b e} & =\left\{u_{1}, \ldots, u_{l}\right\}, & Y^{c e} & =\left\{v_{1}, \ldots, v_{l}\right\}, \\
X^{b e} & =\left\{t_{1}, \ldots, t_{k}\right\}, & X^{c e} & =\left\{w_{1}, \ldots, w_{k}\right\}
\end{aligned}
$$

with the following properties:

- $Y^{a} X^{a} Y^{b} X^{b y} X^{b x} Y^{b e} X^{b e}$ is part of a basis of $B$,

- $Y^{a} X^{a} Y^{c} X^{c y} X^{c x} Y^{c e} X^{c e}$ is part of a basis of $C$,

$\bullet Y^{b} X^{b y} X^{b x} Y^{b e} X^{b e} Y^{c} X^{c y} X^{c x} Y^{c e} X^{c e}$ is linearly independent over $A$

$\bullet Y^{e}=\left\{u_{1}+v_{1}, \ldots, u_{l}+v_{l}\right\} \subseteq H$ is a basis of $H$ modulo $(K \cap B)+(K \cap C)$, and

- $Y^{a} Y^{b} Y^{c} Y^{e} X^{a} X^{b y} X^{b x} X^{c y} X^{c x} X^{e}$ is a suitable basis of $K$, where $X^{e}=\left\{t_{1}+w_{1}, \ldots, t_{k}+w_{k}\right\}$. We call it a suitable basis of $K$ that respects $H$. 
We also say that $X^{a} X^{b y} X^{b x} X^{c x} X^{e}$ or $X^{a} X^{b x} X^{c y} X^{c x} X^{e}$ respectively is a suitable extension of $Y^{a} Y^{b} Y^{c} Y^{d}$ for $K$. All such terminology refers to the situation described above.

Lemma 5.1. Assume that $K$ is a minimal extension of $H$ in $D$.

1. If $K+C \neq H+C$ and $K \cap C \neq H \cap C$, then $\delta((K+C) \cap B)<\delta((H+C) \cap B)$.

2. If $K+B \neq H+B$ and $K \cap B \neq H \cap B$, then $\delta((K+B) \cap C)<\delta((H+B) \cap C)$.

3. If $K \cap C=H \cap C, K \cap B \neq H \cap B$, and $K \neq H+(K \cap B)$, then $\delta((K+B) \cap C)<$ $\delta((H+B) \cap C)$.

4. If $K \cap B=H \cap B, K \cap C \neq H \cap C$, and $K \neq H+(K \cap C)$, then $\delta((K+C) \cap B)<$ $\delta((H+C) \cap B)$.

5. If $K \cap C=H \cap C$, then $\delta((K+C) \cap B) \leq \delta((H+C) \cap B)$.

6. If $K \cap B=H \cap B$, then $\delta((K+B) \cap C) \leq \delta((H+B) \cap C)$.

Proof. Assume that $Y=Y^{a} Y^{b} Y^{c} Y^{d}$ is a suitable basis of $H$, and

$$
X=X^{a} X^{b y} X^{b x} X^{c x} X^{e} \quad \text { or } \quad X=X^{a} X^{b x} X^{c y} X^{c x} X^{e}
$$

respectively gives us a suitable extension of $Y$. Let

$$
Y^{a} Y^{b} Y^{c} Y^{e} X^{a} X^{b y} X^{b x} X^{c y} X^{c x} X^{e}
$$

be the corresponding suitable basis for $K$, where $Y^{e}=\left\{u_{1}+v_{1}, \ldots, u_{l}+v_{l}\right\}$ and $X^{e}=\left\{t_{1}+w_{1}, \ldots, t_{k}+w_{k}\right\}$. Using this suitable basis, we can formulate the assertions of the lemma in the following way:

1. If $\emptyset \neq X^{b x} X^{e} \neq X$, then

$$
\begin{aligned}
& \operatorname{dim}\left(N\left(\left\langle X^{b x}\left\{t_{1}, \ldots, t_{k}\right\} Y^{b} X^{b y}\left\{u_{1}, \ldots, u_{l}\right\} A\right\rangle\right)\right) \\
& \quad-\operatorname{dim}\left(N\left(\left\langle Y^{b} X^{b y}\left\{u_{1}, \ldots, u_{l}\right\} A\right\rangle\right)\right)>\left|X^{b x}\right|+k .
\end{aligned}
$$

2. If $\emptyset \neq X^{c x} X^{e} \neq X$, then

$$
\begin{aligned}
& \operatorname{dim}\left(N\left(\left\langle X^{c x}\left\{w_{1}, \ldots, w_{k}\right\} Y^{c} X^{c y}\left\{v_{1}, \ldots, v_{l}\right\} A\right\rangle\right)\right) \\
& \quad-\operatorname{dim}\left(N\left(\left\langle Y^{c} X^{c y}\left\{v_{1}, \ldots, v_{l}\right\} A\right\rangle\right)\right)>\left|X^{c x}\right|+k .
\end{aligned}
$$

3. If $X^{b x} X^{e}=X, X^{b x} \neq \emptyset$, and $X^{e} \neq \emptyset$, then

$$
\begin{gathered}
\operatorname{dim}\left(N\left(\left\langle\left\{w_{1}, \ldots, w_{k}\right\} Y^{c}\left\{v_{1}, \ldots, v_{l}\right\} A\right\rangle\right)\right) \\
-\operatorname{dim}\left(N\left(\left\langle Y^{c}\left\{v_{1}, \ldots, v_{l}\right\} A\right\rangle\right)\right)>k .
\end{gathered}
$$

4. If $X^{c x} X^{e}=X, X^{c x} \neq \emptyset$, and $X^{e} \neq \emptyset$, then

$$
\begin{array}{r}
\operatorname{dim}\left(N\left(\left\langle\left\{t_{1}, \ldots, t_{k}\right\} Y^{b}\left\{u_{1}, \ldots, u_{l}\right\} A\right\rangle\right)\right) \\
-\operatorname{dim}\left(N\left(\left\langle Y^{b}\left\{u_{1}, \ldots, u_{l}\right\} A\right\rangle\right)\right)>k .
\end{array}
$$

5. If $X^{b x} X^{e}=X$, then

$$
\begin{gathered}
\operatorname{dim}\left(N\left(\left\langle X^{b x}\left\{t_{1}, \ldots, t_{k}\right\} Y^{b}\left\{u_{1}, \ldots, u_{l}\right\} A\right\rangle\right)\right) \\
-\operatorname{dim}\left(N\left(\left\langle Y^{b}\left\{u_{1}, \ldots, u_{l}\right\} A\right\rangle\right)\right) \geq|X| .
\end{gathered}
$$

6. If $X^{c x} X^{e}=X$, then

$$
\begin{gathered}
\operatorname{dim}\left(N\left(\left\langle X^{c x}\left\{w_{1}, \ldots, w_{k}\right\} Y^{c}\left\{v_{1}, \ldots, v_{l}\right\} A\right\rangle\right)\right) \\
-\operatorname{dim}\left(N\left(\left\langle Y^{c}\left\{v_{1}, \ldots, v_{l}\right\} A\right\rangle\right)\right) \geq|X| .
\end{gathered}
$$


We have rephrased the statements of the lemma in the form above since the proofs use a basic commutator argument.

ad (1) We assume that $\left\{u_{1}^{+}, \ldots, u_{m}^{+}\right\}=X^{b y}\left\{u_{1}, \ldots, u_{l}\right\}$ and $\left\{v_{1}^{+}, \ldots, v_{m}^{+}\right\}=$ $X^{c y}\left\{v_{1}, \ldots, v_{l}\right\}$. By the minimality of $K$ over $H$ and the assumption we have $\operatorname{dim}(N(\langle X Y\rangle))-\operatorname{dim}\left(N\left(\left\langle X^{c x} X^{b y} X^{a} Y\right\rangle\right)\right)>\left|X^{b x}\right|+k$. Let $\Phi_{1}, \ldots, \Phi_{r}$ be a basis of $N\left(\left\langle\left(X \backslash X^{e}\right) Y\right\rangle\right)$ over $N\left(\left\langle\left(X \backslash X^{b x} X^{e}\right) Y\right\rangle\right)$. Let $\Delta_{1}, \ldots, \Delta_{s}$ be a basis of $N(\langle X Y\rangle)$ over $N\left(\left\langle\left(X \backslash X^{e}\right) Y\right\rangle\right)$. Then $\left.r+s\right\rangle\left|X^{b x}\right|+k$. First we describe the $\Phi_{i}$ 's and $\Delta_{i}$ 's as linear combinations of basic commutators with respect to

$$
Y^{a} Y^{b} Y^{c}\left\{u_{1}^{+}, \ldots, u_{m}^{+}\right\}\left\{v_{1}^{+}, \ldots, v_{m}^{+}\right\} X^{a} X^{b x} X^{c x}\left\{t_{1}, \ldots, t_{k}\right\}\left\{w_{1}, \ldots, w_{k}\right\}
$$

This set is part of a basis of $D$. By Lemma $4.3 \Phi_{i}=\Phi_{i}^{b}+\Phi_{i}^{c}$, where $\Phi_{i}^{b}$ is a linear combination of basic commutators over

$$
X^{a} X^{b x}\left\{u_{1}^{+}, \ldots, u_{m}^{+}\right\} Y^{a} Y^{b},
$$

and $\Phi_{i}^{c}$ is a linear combination of basic commutators over

$$
X^{a} X^{c x}\left\{v_{1}^{+}, \ldots, v_{m}^{+}\right\} Y^{a} Y^{c},
$$

and there exists some $I_{i} \in \wedge^{2} A$, such that $\Phi_{i}^{b}+I_{i} \in N(B)$ and $\Phi_{i}^{c}-I_{i} \in N(C)$.

By assumption every non-trivial combination of the $\Phi_{i}$ 's contains a basic commutator $z \wedge b_{x}$, where $b_{x} \in X^{b x}$. Therefore the $\left(\Phi_{i}^{b}+I_{i}\right)$ 's are linearly independent over $N\left(\left\langle Y^{b}\left\{u_{1}^{+}, \ldots, u_{m}^{+}\right\} A\right\rangle\right)$.

Again by Lemma 4.3: $\Delta_{i}=\Delta_{i}^{b}+\Delta_{i}^{c}+\Delta_{i}^{b e}+\Delta_{i}^{c e}$, where $\Delta_{i}^{b}$ is a linear combination of basic commutators over $X^{a} X^{b x} Y^{a} Y^{b}\left\{u_{1}^{+}, \ldots, u_{m}^{+}\right\}, \Delta_{i}^{c}$ is a linear combination of basic commutators over $X^{a} X^{c x} Y^{a} Y^{c}\left\{v_{1}^{+}, \ldots, v_{m}^{+}\right\}, \Delta_{i}^{b e}$ is a linear combination of basic commutators $a \wedge t_{i}$ where $a \in Y^{a} X^{a}, \Delta_{i}^{c e}$ is obtained from $\Delta_{i}^{b e}$ by replacing $t_{i}$ by $w_{i}$, and there exists some $J_{i} \in \wedge^{2} A$ with $\Delta_{i}^{b}+\Delta_{i}^{b e}+J_{i} \in N(B)$ and $\Delta_{i}^{c}+\Delta_{i}^{c e}-J_{i} \in$ $N(C)$.

Since the $\Delta_{i}$ 's are linearly independent over $N\left(\left\langle\left(X \backslash X^{e}\right) Y\right\rangle\right)$, every non-trivial linear combination of them contains some basic commutator $a \wedge t_{i}$. It follows that $\left\{\Phi_{i}^{b}+I_{i}: 1 \leq i \leq r\right\} \cup\left\{\Delta_{i}^{b e}+\Delta_{i}^{b}+J_{i}: 1 \leq i \leq s\right\}$ is linearly independent over $N\left(\left\langle Y^{b}\left\{u_{1}^{+}, \ldots, u_{m}^{+}\right\} A\right\rangle\right)$, as desired.

ad (2) Obviously (2) is proved analogously to (1).

ad (5) Also for (5) we use the method of (1). For the start, minimality gives $\operatorname{dim}(N(\langle X Y\rangle))-\operatorname{dim}(N(\langle Y\rangle))=|X|$.

ad (6) Again (6) is shown as (5).

ad (3) We use the notation of (1). By the minimality we get $s>\left|X^{e}\right|=k$. The linear independence of $\Delta_{1}, \ldots, \Delta_{s}$ over $N\left(\left\langle Y X^{b x}\right\rangle\right)$ implies that every non-trivial linear combination of them contains the basic commutators $a \wedge t_{i}$ and $a \wedge w_{i}$ for some $i$ and $a \in Y^{a} X^{a}$. Therefore the $\left(\Delta_{i}^{c}+\Delta^{c e}-J_{i}\right)$ 's are linearly independent over $N\left(\left\langle Y^{c}\left\{v_{1}^{+}, \ldots, v_{m}^{+}\right\} A\right\rangle\right)$, as desired.

ad (4) We show (4) similarly as (3).

Lemma 5.2. Let $K$ be a minimal extension of $H$ in $D$. Assume that $H \subseteq A$. Let $K_{1}, \ldots, K_{h}$ be a set of realizations of $K$ over $H$. Suppose that there is no realization $K_{i}$ of this minimal extension over $H$ with $\left(K_{i} \cap B\right)+\left(K_{i} \cap C\right)=H$. Then either all realizations $K_{i}$ are in $B$ or all realizations $K_{i}$ are in $C$. In the first case $\left\langle A K_{i}\right\rangle=B$ or $K_{i} \subseteq A$.

Proof. If for some $i$ with $1 \leq i \leq h, K_{i}$ is contained neither in $B$ nor $C$, then either $K_{i} \cap C \neq H$ and 5.1 (1) yields $\delta\left(\left(K_{i}+C\right) \cap B\right)<\delta(A)$, contradicting the 
selfsufficiency of $A$ in $B$, or else $K_{i} \cap C=H$ and 5.1 (3) yields $\delta\left(\left(K_{i}+B\right) \cap C\right)<\delta(A)$, which contradicts the $(\operatorname{dim}(B / A))$-selfsufficiency of $A$ in $C$, as $K_{i} \cap C \subseteq A$.

Thus for each $i$ we have $K_{i} \subseteq B$ or $K_{i} \subseteq C$. If $K_{i} \subseteq B$ and $K_{i}$ is not contained in $A$, then again by 5.1 (1) we have $K_{i} \cap C=H$, and by 5.1 (5) $\delta\left(\left\langle A K_{i}\right\rangle\right) \leq \delta(A)$, forcing $\left\langle A K_{i}\right\rangle=B$ by the minimality of $B$ over $A$.

Now suppose toward a contradiction that $\left\langle A K_{i}\right\rangle=B$ and that for some $j K_{j} \not{Z}$ $B$. Then $K_{j} \leq C$, and by $5.1(2)$ and the $(\operatorname{dim}(B / A))$-selfsufficiency of $A$ in $C$ we find that $K_{j} \cap B=H$, and then by 5.1 (6) we get $\delta\left(\left\langle K_{j} A\right\rangle\right)=\delta(A)$. Let $\Phi_{1}, \ldots, \Phi_{r}$ be a basis of $N\left(K_{i}\right)$ modulo $N(H)$, where $r=\operatorname{dim}(K / H)$. Then $\Phi_{1}, \ldots, \Phi_{r}$ are linearly independent modulo $\wedge^{2} A$. Let $f$ be an isomorphism of $K_{i}$ with $K_{j}$ over $H$. Then $f\left(\Phi_{1}\right), \ldots, f\left(\Phi_{r}\right)$ are linearly independent over $\wedge^{2} H$ and hence over $\wedge^{2} A$. As $\delta\left(\left\langle K_{j} A\right\rangle\right)=\delta(A)$, we find that $f\left(\Phi_{1}\right), \ldots, f\left(\Phi_{r}\right)$ form a basis for $N\left(\left\langle A K_{j}\right\rangle\right)$ over $N(A)$, and hence $f$ extends to an isomorphic embedding of $\left\langle A K_{i}\right\rangle=B$ into $C$, a contradiction.

Lemma 5.3. Assume that $M$ is a structure in $\mathcal{S}$ that satisfies $(\Sigma 3)$. Let $K_{1}, \ldots, K_{h}$ be realizations of the minimal extension $K_{1}$ of $H$, where $H$ is selfsufficient in $M$. Furthermore suppose that $\left.K_{i} \nsubseteq \backslash \bigcup_{1 \leq j<i} K_{j}\right\rangle$ for every $i$ with $1 \leq i \leq h$. Then for every $i$ we have: $K_{1}, \ldots, K_{i}$ are linearly independent over $H$ and $\delta\left(\left\langle\bigcup_{1 \leq j \leq i} K_{j}\right\rangle\right)=$ $\delta(H)$.

Proof. We use induction on $i$. For $i=1$ the assertion is clear. Assume that the assertion is proved for all $j<i$. Then $\delta\left(\left\langle\bigcup_{1 \leq j<i} K_{j}\right\rangle\right)=\delta(H)$ and $K_{1}, \ldots, K_{i-1}$ are linearly independent over $H$. By Lemma 3.4

$$
\delta\left(\left\langle\bigcup_{1 \leq j \leq i} K_{j}\right\rangle\right) \leq \delta\left(\left\langle\bigcup_{1 \leq j<i} K_{j}\right\rangle\right)+\delta\left(K_{i}\right)-\delta\left(K_{i} \cap\left\langle\bigcup_{1 \leq j<i} K_{j}\right\rangle\right) .
$$

By induction and the assumption of the lemma we obtain

$$
\delta\left(\left\langle\bigcup_{1 \leq j \leq i} K_{j}\right\rangle\right) \leq \delta(H)+\delta(H)-\delta\left(K_{i} \cap\left\langle\bigcup_{1 \leq j<i} K_{j}\right\rangle\right)
$$

Obviously $H \subseteq\left(K_{i} \cap\left\langle\bigcup_{1<j<i} K_{j}\right\rangle\right) \subseteq K_{i}$. Let us assume that $K_{1}, \ldots, K_{i}$ are not linearly independent over $\bar{H}$. This and the linear independence of $K_{1}, \ldots, K_{i-1}$ over $H$ imply the first of the following two inequations: $H \neq K_{i} \cap\left\langle\bigcup_{1 \leq j<i} K_{j}\right\rangle \neq K_{i}$. The second is the assumption of the lemma. By the minimality of $K_{i}$ over $H$, $\delta\left(K_{i} \cap\left\langle\bigcup_{1 \leq j<i} K_{j}\right\rangle\right)>\delta(H)$. By $(1) \delta\left(\left\langle\bigcup_{1 \leq j \leq i} K_{j}\right\rangle\right)<\delta(H)$, a contradiction to the selfsufficiency of $H$ in $M$. Hence $K_{1}, \ldots, \bar{K}_{i}$ are linearly independent over $H$ and we have $K_{i} \cap\left\langle\bigcup_{1 \leq j<i} K_{j}\right\rangle=H$. By (1) $\delta\left(\left\langle\bigcup_{1 \leq j \leq i} K_{j}\right\rangle\right) \leq \delta(H)$, and by the selfsufficiency of $H$ in $M$ we get equality, as desired.

\section{The Second Amalgamation Theorem}

Let $M$ be any structure in $\mathcal{S}$ that fulfills ( $\Sigma 3$ ). Assume that $H \subseteq K \subseteq M$, $H$ and $K$ are finite subspaces, and $K$ is a minimal extension of $H$. Suppose that $K_{1}, \ldots, K_{h}$ is a sequence of realizations of $K=K_{1}$ over $H$. Let $X_{i}$ be a basis of $K_{i}$ over $H$. We say that $K_{1}, \ldots, K_{h}$ (or $X_{1}, \ldots, X_{h}$ respectively) are in free composition over $H$, if $X_{1}, \ldots, X_{h}$ are linearly independent over $H$ and if

$$
\delta\left(\left\langle\bigcup_{1 \leq j \leq h} K_{j}\right\rangle\right)=\delta(H)
$$


If $H$ is selfsufficient in $M$ and $\left.K_{i} \nsubseteq \backslash \bigcup_{1 \leq j<i} K_{j}\right\rangle$ for all $1 \leq i \leq h$, then $K_{1}, \ldots, K_{h}$ are in free composition over $H$, by Lemma 5.3. Let $X_{i}$ be $\left\{x_{1}^{i}, \ldots, x_{r}^{i}\right\}$ for $1 \leq i \leq$ $h$. We call $X_{1}, \ldots, X_{h}$ a sequence of compatible bases, if the map $f_{k}$ defined by $f_{k}\left(x_{j}^{1}\right)=x_{j}^{k}$, and $f(y)=y$ for $y \in H$ induces an isomorphism of the $\mathcal{S}$ - structures $K_{1}$ onto $K_{k}$ over $H$. Of course a compatible system of bases $X_{1}, \ldots, X_{h}$ over $H$ exists for every sequence of realizations $K_{1}, \ldots, K_{h}$ of a minimal extension $K_{1}$ over $H$. Suppose such a system is given. Let $Y$ be a basis of $H$. Let $\Phi$ be any element of $\wedge^{2} K_{1}$, presented as a linear combination of basic commutators over $Y X_{1}$. Then $f_{k}(\Phi)$ denotes its image in $\wedge^{2} K_{k}$, replacing each $x_{j}^{1}$ by $x_{j}^{k}$.

Lemma 6.1. Assume that $K_{1}$ is a minimal extension of $H$ in $M$. Let $X_{1}, \ldots, X_{h}$ be a sequence of compatible bases of realizations $K_{1}, \ldots, K_{h}$ of $K_{1}$ over $H$ that are in free composition over $H$. Let $\sigma$ be any permutation of $1, \ldots, h$.

1. For every $i$ with $1 \leq i \leq h$, we have that $\left\langle\bigcup_{1 \leq j \leq i} K_{\sigma(j)}\right\rangle$ is a minimal extension of $\left\langle\bigcup_{1 \leq j<i} K_{\sigma(j)}\right\rangle$.

2. Let $f_{\sigma}$ be the map $f_{\sigma}\left(x_{j}^{i}\right)=x_{j}^{\sigma(i)}$ and $f_{\sigma}(y)=y$ for $y \in H$. Then $f_{\sigma}$ can be extended to an isomorphism of $\left\langle H X_{1} \ldots X_{h}\right\rangle$ onto $\left\langle H X_{1} \ldots X_{h}\right\rangle$ that induces an isomorphism of the corresponding structures in $\mathcal{S}$.

Proof. As above let $Y$ be a basis of $H$. Let us consider all permutations $\sigma$ of $1, \ldots, h$ and all $i$ with $1 \leq i \leq h$. By the minimality of $K_{1}$ over $H$ there are $\Phi_{1}, \ldots, \Phi_{r}(r=|X|)$ in $N\left(\left\langle Y X_{1}\right\rangle\right)$ such that $\Phi_{1}, \ldots, \Phi_{r}$ freely generate $N\left(\left\langle Y X_{1}\right\rangle\right)$ over $N(H)$. We consider $\Phi_{1}, \ldots, \Phi_{r}$ as linear combinations of basic commutators over $Y X_{1}$. Then every non-trivial linear combination of them contains some $z \wedge x_{j}^{1}$ for some $1 \leq j \leq r$ and $z \in Y X_{1}$. Hence $f_{\sigma(i)}\left(\Phi_{1}\right), \ldots, f_{\sigma(i)}\left(\Phi_{r}\right)$ in $N\left(\left\langle Y X_{\sigma(i)}\right\rangle\right)$ are linearly independent over $N(H)$ and also in $N\left(\left\langle Y \bigcup_{1<j<i} X_{\sigma(j)}\right\rangle\right)$ over $N\left(\left\langle Y \bigcup_{1 \leq j<i} X_{\sigma(j)}\right\rangle\right)$. From this and $\delta\left(\sum_{1 \leq j \leq h} K_{j}\right)=\delta(H)$ it follows that $\delta\left(\left\langle Y \bigcup_{1 \leq j \leq i} X_{\sigma(j)}\right\rangle\right)=\delta(H)$. Hence $f_{\sigma(i)}\left(\Phi_{1}\right), \ldots, f_{\sigma(i)}\left(\Phi_{r}\right)$ freely generate $N\left(\left\langle Y \bigcup_{1 \leq j \leq i}^{\leq j \leq i} X_{\sigma(j)}\right\rangle\right)$ over $N\left(\left\langle Y \bigcup_{1 \leq j<i} X_{\sigma(j)}\right\rangle\right)$. This implies both assertions of the lemma.

As above, let $K_{1}, \ldots, K_{h}$ be a sequence of realizations of the minimal extension $K_{1}$ of $H$ in $M$. We assume that they are in free composition over $H$. Again let $X_{j}=\left\{x_{1}^{j}, \ldots, x_{r}^{j}\right\}$ for $1 \leq j \leq h$ be a system of compatible bases. Let $\sigma$ be any permutation of $1, \ldots, h$. As above in Lemma 6.1 let $f_{\sigma}$ be the corresponding automorphism of the $\mathcal{S}$-structure generated by $H X_{1} \ldots X_{h}$ in $M$. We write $f_{i}$ instead of $f_{\sigma}$, if $\sigma$ is the permutation that only exchanges 1 and $i$. Let $\Phi_{1}, \ldots, \Phi_{r}$ be free generators of $N\left(\left\langle H X_{1}\right\rangle\right)$ over $N(H)$. Then $f_{i}\left(\Phi_{1}\right), \ldots, f_{i}\left(\Phi_{r}\right)$ freely generate $N\left(H X_{1} X_{i}\right)$ over $N\left(H X_{1}\right)$. But also $f_{i}\left(\Phi_{1}\right)-\Phi_{1}, \ldots, f_{i}\left(\Phi_{r}\right)-\Phi_{r}$ do this. By definition $f_{i}\left(\Phi_{j}\right)-\Phi_{j}$ for $1<i \leq h$ has the following form, where $0 \leq \alpha_{t s}^{j}<p$ and $y_{t}^{j} \in K$ :

$$
\sum_{s<t} \alpha_{s t}^{j}\left(\left(x_{s}^{i} \wedge x_{t}^{i}\right)-\left(x_{s}^{1} \wedge x_{t}^{1}\right)\right)+\sum_{t}\left(y_{t}^{j} \wedge\left(x_{t}^{i}-x_{t}^{1}\right)\right) .
$$

Note that $\alpha_{s t}^{j}$ and $y_{t}^{j}$ do not depend on $i$. If we apply a suitable permutation to the elements of $X_{j}$ uniformly for all $j$, then we find some $k(1 \leq k \leq r)$ such that $\alpha_{t s_{1}}^{j}=0=\alpha_{s_{2} t}^{j}$ for all $j$ and all $s_{1}, s_{2}$ if and only if $k<t$. We introduce the following notation: 
For $1 \leq i \leq h, X_{i}^{+}=\left\{x_{1}^{i}, \ldots, x_{k}^{i}\right\}$ and for $2 \leq i \leq h, X_{i}^{-}=\left\{z_{k+1}^{i}, \ldots, z_{r}^{i}\right\}$, where $z_{t}^{i}=x_{t}^{i}-x_{t}^{1}$. We choose a linearly independent set $Y \subseteq H$ such that

$$
\langle Y\rangle=\left\langle\left\{y_{t}^{j}: 1 \leq t \leq r, 1 \leq j \leq r\right\}\right\rangle
$$

Then the sequence $Y X_{1}^{+} X_{2}^{+} X_{2}^{-} \ldots X_{h}^{+} X_{h}^{-}$has the following properties:

(S1): For $2 \leq i \leq h$ the vector space $\left\langle Y X_{1}^{+} X_{2}^{+} X_{2}^{-} \ldots X_{i}^{+} X_{i}^{-}\right\rangle$is a minimal extension of $\left\langle Y X_{1}^{+} X_{2}^{+} X_{2}^{-} \ldots X_{i-1}^{+} X_{i-1}^{-}\right\rangle$. Also $N\left(\left\langle Y X_{1}^{+} X_{2}^{+} X_{2}^{-} \ldots X_{i}^{+} X_{i}^{-}\right\rangle\right)$ is freely generated by $\Phi_{1}^{i}, \ldots, \Phi_{r}^{i}$ over $N\left(\left\langle Y X_{1}^{+} X_{2}^{+} X_{2}^{-} \ldots X_{i-1}^{+} X_{i-1}^{-}\right\rangle\right)$, where $\Phi_{j}^{i}$ is of the form

$$
\sum_{1 \leq s<t \leq k} \alpha_{s t}^{j}\left(\left(x_{s}^{i} \wedge x_{t}^{i}\right)-\left(x_{s}^{1} \wedge x_{t}^{1}\right)\right)+\sum_{1 \leq t \leq k}\left(y_{t}^{j} \wedge\left(x_{t}^{i}-x_{t}^{1}\right)\right)+\sum_{k<t \leq r}\left(y_{t}^{j} \wedge z_{t}^{i}\right) .
$$

$\alpha_{t s}^{j}$ and $y_{t}^{j}$ do not depend on $i$, for every $t \leq k$ there is some $s \leq k$ and some $j$ such that $\alpha_{t s}^{j} \neq 0$ or $\alpha_{s t}^{j} \neq 0$, and $\langle Y\rangle=\left\langle\left\{y_{t}^{j}: 1 \leq t \leq r, 1 \leq j \leq r\right\}\right\rangle$.

(S2): For every permutation $\sigma$ of $1, \ldots, h$ with $\sigma(1)=1$ define $f_{\sigma}(y)=y$, $f_{\sigma}\left(x_{t}^{i}\right)=x_{t}^{\sigma(i)}$ for $1 \leq t \leq k$ and $f_{\sigma}\left(z_{t}^{i}\right)=z_{t}^{\sigma(i)}$ for $k<t \leq r$. Then $f_{\sigma}$ induces an automorphism of $\left\langle Y X_{1}^{+} X_{2}^{+} X_{2}^{-} \ldots X_{h}^{+} X_{h}^{-}\right\rangle$and the corresponding structure in $\mathcal{S}$.

(S3): Let $1<j$. For $1 \leq t \leq k$ define $f_{j}\left(x_{t}^{i}\right)=x_{t}^{i}$ for $2 \leq i \leq h$ and $i \neq j$, $f_{j}\left(x_{t}^{1}\right)=x_{t}^{j}$, and $f_{j}\left(x_{t}^{j}\right)=x_{t}^{1}$. For $k<t \leq r$ define $f_{j}\left(z_{t}^{i}\right)=z_{t}^{i}-z_{t}^{j}$ for $2 \leq i \leq h$ and $i \neq j$, and $f_{j}\left(z_{t}^{j}\right)=-z_{t}^{j}$. Then $f_{j}$ induces an automorphism of $\left\langle Y X_{1}^{+} X_{2}^{+} X_{2}^{-} \ldots X_{h}^{+} X_{h}^{-}\right\rangle$and the corresponding structure in $\mathcal{S}$.

(S1) was already proved above. (S2) follows from Lemma 6.1. To obtain (S3) we repeat our considerations with $X_{j}$ and $X_{1}$ exchanged, and compare the result with the situation in (S1) via Lemma 6.1.

Now we have to realize that there are many ways to choose a sequence of compatible bases $X_{1}, \ldots, X_{h}$ of $K_{1}, \ldots, K_{h}$ over $H$. Among all such, we choose one that gives us first a minimal $k$ in (S1), and then a minimal $\langle Y\rangle$ in (S1). Let $X_{i}^{+}-X_{1}^{+}$ be $\left\{x_{t}^{i}-x_{t}^{1}: 1 \leq t \leq k\right\}$.

if

We call a vector space automorphism $f$ of $\left\langle Y X_{1}^{+} X_{2}^{+} X_{2}^{-} \ldots X_{h}^{+} X_{h}^{-}\right\rangle$well-tolerated

(a) $f(y)=y$ for $y \in Y, f\left(\left\langle Y X_{i}^{+}\right\rangle\right)=\left\langle Y X_{i}^{+}\right\rangle, f\left(\left\langle\left(X_{i}^{+}-X_{1}^{+}\right) X_{i}^{-}\right\rangle\right)=\left\langle\left(X_{i}^{+}-\right.\right.$ $\left.\left.X_{1}^{+}\right) X_{i}^{-}\right\rangle$,

(b) for $1 \leq i, j \leq h$ the equality $f\left(x_{t}^{i}\right)=y+\sum_{1 \leq s \leq k} \gamma_{s} x_{s}^{i}$ holds if and only if

$$
f\left(x_{t}^{j}\right)=y+\sum_{1 \leq s \leq k} \gamma_{s} x_{s}^{j} \quad \text { where } y \in Y,
$$

(c) for $2 \leq i, j \leq r$ the equality $f\left(z_{t}^{i}\right)=\sum_{1 \leq s \leq k} \eta_{s}\left(x_{s}^{i}-x_{s}^{1}\right)+\sum_{k<s \leq r} \eta_{s} z_{s}^{i}$ holds if and only if

$$
f\left(z_{t}^{j}\right)=\sum_{1 \leq s \leq k} \eta_{s}\left(x_{s}^{j}-x_{s}^{1}\right)+\sum_{k<s \leq r} \eta_{s} z_{s}^{j} .
$$

Note that a well-tolerated automorphism $f$ of $\left\langle Y X_{1}^{+} X_{2}^{+} X_{2}^{-} \ldots X_{h}^{+} X_{h}^{-}\right\rangle$as above can be extended to a vector space automorphism $g$ of $\left\langle Y X_{1}, \ldots, X_{h}\right\rangle$ with $g\left(\left\langle Y X_{i}\right\rangle\right)$ $=\left\langle Y X_{i}\right\rangle$. By our choice of $Y, X_{1}, \ldots, X_{h}$ we obtain: 
(S4): For every well-tolerated automorphism $f$ of $\left\langle Y X_{1}^{+} X_{2}^{+} X_{2}^{-} \ldots X_{h}^{+} X_{h}^{-}\right\rangle$the sequence $Y f\left(X_{1}^{+}\right) f\left(X_{2}^{+}\right) f\left(X_{2}^{-}\right) \ldots f\left(X_{h}^{+}\right) f\left(X_{h}^{-}\right)$again satisfies (S1) with the same $k$ and $\langle Y\rangle$, (S2), and (S3).

Now let $Y X_{1}^{+} X_{2}^{+} X_{2}^{-} \ldots X_{h}^{+} X_{h}^{-}$be any sequence with $X_{i}^{+}=\left\{x_{1}^{i}, \ldots, x_{k}^{i}\right\}$ and $X_{i}^{-}=\left\{z_{k+1}^{i}, \ldots, z_{r}^{i}\right\}$. We call $Y X_{1}^{+} X_{2}^{+} X_{2}^{-} \ldots X_{h}^{+} X_{h}^{-}$a reduced k-sequence, if $Y X_{1}^{+} X_{2}^{+} X_{2}^{-} \ldots X_{h}^{+} X_{h}^{-}$is linearly independent and $Y X_{1}^{+} X_{2}^{+} X_{2}^{-} \ldots X_{h}^{+} X_{h}^{-}$fulfills (S1), (S2), (S3), and (S4).

Let $(\Sigma 4)^{m}$ be the following property:

$(\Sigma 4)^{m}$ : For every reduced $k$-sequence $Y X_{1}^{+} X_{2}^{+} X_{2}^{-} \ldots X_{h}^{+} X_{h}^{-}$in $M$ with $k+$ $|Y|, r<m$ we obtain

$$
h \leq 2(\max \{k+|Y|, r\})+1 .
$$

Furthermore we define:

$(\Sigma 4)$ : For every reduced $k$-sequence $Y X_{1}^{+} X_{2}^{+} X_{2}^{-} \ldots X_{h}^{+} X_{h}^{-}$we obtain $h \leq$ $2(\max \{k+|Y|, r\})+1$.

In the following lemma we summarize some trivial facts about reduced $k$-sequences:

Lemma 6.2. Let $Y X_{1}^{+} X_{2}^{+} X_{2}^{-} \ldots X_{h}^{+} X_{h}^{-}$be a reduced $k$-sequence.

1. $|Y| \leq r^{2}$.

2. Linear combinations of the relations $\Phi_{1}^{i}, \ldots, \Phi_{r}^{i}$ in (S1) are again of the form described in (S1).

3. The composition of two well-tolerated automorphisms is again well-tolerated. Therefore we can iterate (S4).

4. Assume that $M$ fulfills $(\Sigma 4)$. If $K_{1}, \ldots, K_{h}$ are realizations of a minimal extension of $H$ that are in free composition over $H$, then $h \leq 2 \operatorname{dim}\left(K_{1}\right)+1$.

Proof. To prove (4) we construct a reduced $k$-sequence from $H$ and $K_{1}, \ldots, K_{h}$ as described above. Then the assertion follows.

By Lemma 6.2.3 the application of well-tolerated automorphisms transforms a reduced $k$-system into a reduced $k$-system again. As above let $A, B$, and $C$ be finite structures in $\mathcal{S}$ that fulfill $(\Sigma 3)$. Assume that $A$ is a common substructure of $B$ and $C$, and that $A$ is selfsufficient in $B$ and $(\operatorname{dim}(B / A)+2+l)$-selfsufficient in $C$ for some $l$. Let $D$ be $B \star_{A} C$. By Theorem 4.1 $D$ fulfills $(\Sigma 3)^{0}$. Often we assume, furthermore, that $B$ is a minimal extension of $A$ that is not realized in $C$. Then $D$ fulfills $(\Sigma 3)$.

Lemma 6.3. Assume $A, B, C$, and $D=B \star_{A} C$ in $\mathcal{S}$ are chosen as described above such that $D$ fulfills $(\Sigma 3)^{0}$. Let $Y X_{1}^{+} X_{2}^{+} X_{2}^{-} \ldots X_{h}^{+} X_{h}^{-}$be a reduced $k$-sequence in $D$ with $0<k$. Assume that $Y$ is a suitable basis of $\langle Y\rangle$.

1. If $\left\langle Y X_{1}^{+} X_{2}^{+}\right\rangle \cap C=\langle Y\rangle \cap C$, then $\left\langle Y X_{1}^{+} X_{2}^{+}\right\rangle=\langle Y\rangle+\left(\left\langle Y X_{1}^{+} X_{2}^{+}\right\rangle \cap B\right)$.

2. If $\left\langle Y X_{1}^{+}\right\rangle \cap C=\langle Y\rangle \cap C$, and $\left\langle Y X_{1}^{+} X_{2}^{+}\right\rangle \cap B=\left\langle Y X_{1}^{+}\right\rangle \cap B$, then $\left\langle Y X_{1}^{+}\right\rangle=$ $\langle Y\rangle+\left(\left\langle Y X_{1}^{+}\right\rangle \cap B\right),\left\langle Y X_{2}^{+}\right\rangle=\langle Y\rangle+\left(\left\langle Y X_{2}^{+}\right\rangle \cap C\right)$, and $\left\langle Y X_{1}^{+} X_{2}^{+}\right\rangle=\left\langle Y X_{1}^{+}\right\rangle+$ $\left(\left\langle Y X_{1}^{+} X_{2}^{+}\right\rangle \cap C\right)$.

3. If $\left\langle Y X_{1}^{+} X_{2}^{+}\right\rangle \cap B=\langle Y\rangle \cap B$, then $\left\langle Y X_{1}^{+} X_{2}^{+}\right\rangle=\langle Y\rangle+\left(\left\langle Y X_{1}^{+} X_{2}^{+}\right\rangle \cap C\right)$.

4. If $\left\langle Y X_{1}^{+}\right\rangle \cap B=\langle Y\rangle \cap B$ and $\left\langle Y X_{1}^{+} X_{2}^{+}\right\rangle \cap C=\left\langle Y X_{1}^{+} X_{2}^{+}\right\rangle \cap C$, then $\left\langle Y X_{1}^{+}\right\rangle=$ $\langle Y\rangle+\left(\left\langle Y X_{1}^{+}\right\rangle \cap C\right),\left\langle Y X_{2}^{+}\right\rangle=\langle Y\rangle+\left(\left\langle Y X_{2}^{+}\right\rangle \cap B\right)$, and $\left\langle Y X_{1}^{+} X_{2}^{+}\right\rangle=\left\langle Y X_{1}^{+}\right\rangle+$ $\left(\left\langle Y X_{1}^{+} X_{2}^{+}\right\rangle \cap B\right)$. 
Proof. Since we use bases in our proofs, we write the lemma in the language of suitable bases:

1. Assume that $Y S_{1}^{+b x} S_{1}^{+e}$ is a suitable basis for $\left\langle Y X_{1}^{+}\right\rangle$. If $Y S_{2}^{+b x} S_{2}^{+e}$ is a suitable basis for $\left\langle Y X_{2}^{+}\right\rangle$and $Y S_{1}^{+b x} S_{1}^{+e} Z_{2}^{+b x} Z_{2}^{+e}$ is a suitable basis for $\left\langle Y X_{1}^{+} X_{2}^{+}\right\rangle$, then $S_{1}^{+e}=S_{2}^{+e}=Z_{2}^{+e}=\emptyset$.

2. Assume that $Y S_{1}^{+b x} S_{1}^{+e}$ is a suitable basis for $\left\langle Y X_{1}^{+}\right\rangle$. If $Y S_{2}^{+c x} S_{2}^{+e}$ is a suitable basis for $\left\langle Y X_{2}^{+}\right\rangle$and $Y S_{1}^{+b x} S_{1}^{+e} Z_{2}^{+c x} Z_{2}^{+e}$ is a suitable basis for $\left\langle Y X_{1}^{+} X_{2}^{+}\right\rangle$, then $S_{1}^{+e}=S_{2}^{+e}=Z_{2}^{+e}=\emptyset$.

3. Assume that $Y S_{1}^{+c x} S_{1}^{+e}$ is a suitable basis for $\left\langle Y X_{1}^{+}\right\rangle$. If $Y S_{2}^{+c x} S_{2}^{+e}$ is a suitable basis for $\left\langle Y X_{2}^{+}\right\rangle$and $Y S_{1}^{+c x} S_{1}^{+e} Z_{2}^{+c x} Z_{2}^{+e}$ is a suitable basis for $\left\langle Y X_{1}^{+} X_{2}^{+}\right\rangle$, then $S_{1}^{+e}=S_{2}^{+e}=Z_{2}^{+e}=\emptyset$.

4. Assume that $Y S_{1}^{+c x} S_{1}^{+e}$ is a suitable basis for $\left\langle Y X_{1}^{+}\right\rangle$. If $Y S_{2}^{+b x} S_{2}^{+e}$ is a suitable basis for $\left\langle Y X_{2}^{+}\right\rangle$and $Y S_{1}^{+c x} S_{1}^{+e} Z_{2}^{+b x} Z_{2}^{+e}$ is a suitable basis for $\left\langle Y X_{1}^{+} X_{2}^{+}\right\rangle$, then $S_{1}^{+e}=S_{2}^{+e}=Z_{2}^{+e}=\emptyset$.

We show (1) and (2). The proofs of (3) and (4) are similar, since we do not use that $A$ is selfsufficient in $B$ and $(\operatorname{dim}(B / A)+2+l)$-selfsufficient in $C$.

We start with the proof of (1). First we assume that $Z_{2}^{+e} \neq \emptyset$ and show a contradiction. We rewrite (S1) with respect to $Y S_{1}^{+b x} S_{1}^{+e} Z_{2}^{+b x} Z_{2}^{+e}$ to get a contradiction with the help of Lemma 4.3. First we apply several well-tolerated automorphisms of $\left\langle Y X_{1}^{+} X_{2}^{+} X_{2}^{-} \ldots X_{h}^{+} X_{h}^{-}\right\rangle$to make this easier.

By (S4) we can assume w.l.o.g. that

(1) $X_{2}^{+}=S_{2}^{+b x} S_{2}^{+e}$.

Then there exists $k_{0}$ such that $1 \leq k_{0} \leq k$ and $\left\{x_{1}^{2}, \ldots, x_{k_{0}}^{2}\right\}=S_{2}^{+b x}$. Using the notation of (S3) we have $x_{i}^{1}=f_{2}^{-1}\left(x_{i}^{2}\right)$. Using (S4) for a suitable well-tolerated automorphism, we can assume that (1) remains true and w.l.o.g.:

(2) For every $i$ with $1 \leq i \leq k_{0}$ either $x_{i}^{1}+w \notin B$ for all $w \in\left\langle Y\left\{x_{1}^{1}, \ldots, x_{i-1}^{1}\right\}\right\rangle$ or there exists some $y_{i} \in\langle Y\rangle$ such that $x_{i}^{1}-y_{i} \in B$.

In the first case we define $u_{i}^{1}=x_{i}^{1}$, and in the second $u_{i}^{1}=x_{i}^{1}-y_{i} . Y\left\{u_{1}^{1}, \ldots, u_{k_{0}}^{1}\right\}$ can be extended to a suitable basis of $\left\langle Y X_{1}^{+}\right\rangle$. By application of (S4) to welltolerated automorphisms of the form $f\left(x_{i}^{j}\right)=x_{i}^{j}-\sum_{l<i} r_{l} x_{l}^{j}-y$, where $y \in\langle Y\rangle$ and $k_{0}<i$, and then of the form of a suitable permutation, we can assume w.l.o.g. that (1) and (2) remain true and:

(3) There exists $k_{1}$ with $k_{0} \leq k_{1} \leq k$ such that $\left\{u_{i}^{1}: 1 \leq i \leq k_{0}, u_{i} \in B\right\} \cup$ $\left\{x_{k_{0}+1}^{1}, \ldots, x_{k_{1}}^{1}\right\}$ can be considered as $S_{1}^{+b x}$ and $\left\{u_{i}^{1}: 1 \leq i \leq k_{0}, u_{i} \notin B\right\}$ $\cup\left\{x_{k_{1}+1}^{1}, \ldots, x_{k}^{1}\right\}$ as $S_{1}^{+e}$.

For $k_{0} \leq i \leq k$ we define $u_{i}^{1}=x_{i}^{1}$. Then $S_{1}^{+b x}$ can be chosen as the sequence of all $u_{i}^{1}$ with $u_{i}^{1} \in B$ and $S_{1}^{+e}$ as the sequence of all $u_{i}^{1}$ with $u_{i}^{1} \notin B$. We have $X_{2}^{+}=S_{2}^{+b x} S_{2}^{+e}$. But $X_{1}^{+} \neq S_{1}^{+b x} S_{1}^{+e}$ is possible. To obtain equality as sets we have to add the $y_{i}$ 's, as defined above in (2). Now we want to extend $Y S_{1}^{+b x} S_{1}^{+e}$ to a suitable basis of $\left\langle Y X_{1}^{+} X_{2}^{+}\right\rangle$. By assumption of the lemma $\left\langle Y X_{1}^{+} X_{2}^{+}\right\rangle$has a suitable basis of the form $Y S_{1}^{+b x} S_{1}^{+e} Z_{2}^{+b x} Z_{2}^{+e}$. Therefore

(4) $Y S_{1}^{b x} S_{1}^{+e}\left\{x_{1}^{2}, \ldots, x_{k_{0}}^{2}\right\}$ is an initial part of a suitable basis of $\left\langle Y X_{1}^{+} X_{2}^{+}\right\rangle$.

We define $u_{i}^{2}=x_{i}^{2}$ for $1 \leq i \leq k_{0}$. For $k_{0}<i$ we define either

(a) $u_{i}^{2}=x_{i}^{2}$, if $x_{i}^{2}$ is not in $B$ modulo $\left\langle Y S_{1}^{+e}\left\{u_{j}^{2}: j<i\right\}\right\rangle$ or otherwise 
(b) $u_{i}^{2}=x_{i}^{2}-w_{i}^{+}$, where $u_{i}^{2} \in B \backslash A$ and $w_{i}^{+} \in\left\langle Y^{c} Y^{d} S_{1}^{+e}\left\{u_{l}^{2}: l<i, u_{l}^{2} \notin B\right\}\right\rangle$.

Since by (3) $x_{i}^{1}=u_{i}^{1} \in S_{1}^{+b x}$ for $k_{0}<i \leq k_{1}$ and $x_{i}^{1}=u_{i}^{1} \in S_{1}^{+e}$ for $k_{1}<i \leq k$, we can apply (S4) again for well-tolerated automorphisms defined by $f\left(x_{i}^{j}\right)=x_{i}^{j}-$ $\sum_{k_{0}<l<i} r_{l} x_{l}^{j}$. Then (1), (2), (3), and (4) remain true, and

$$
\text { in case (b) } w_{i}^{+} \in\left\langle Y^{c} Y^{d} S_{1}^{+e}\right\rangle \text {. }
$$

Now we can regard $\left\{u_{i}^{j}: j \in\{1,2\}, 1 \leq i \leq k\right\}$ as another enumeration of $S_{1}^{+b x} S_{1}^{+e} Z_{2}^{+b x} Z_{2}^{+e}$. We have the following connections with the basis $Y X_{1}^{+} X_{2}^{+}$:

$u_{i}^{1}=x_{i}^{1}-y_{i}$ for $1 \leq i \leq k_{0}$, where $y_{i} \in\langle Y\rangle$, and $y_{i} \neq 0$ implies $u_{i}^{1} \in S_{1}^{+b x}$

$u_{i}^{2}=x_{i}^{2} \in B$ for $1 \leq i \leq k_{0}$,

$u_{i}^{1}=x_{i}^{1}$ for $k_{0}<i \leq k$, and

$u_{i}^{2}=x_{i}^{2}-w_{i}^{+}$for $k_{0}<i \leq k$, where $w_{i}^{+}=0$ if $u_{i}^{2} \notin B$ and $w_{i}^{+} \in\left\langle Y^{c} Y^{d} S_{1}^{+e}\right\rangle$ otherwise.

First we show that $Z_{2}^{+e}=\emptyset$. That means that all $u_{i}^{2}$ are in $B$. Otherwise there is some $u_{i_{0}}^{2} \notin B$. Then $k_{0}<i_{0}$ and $u_{i_{0}}^{2}$ occurs in the unique representation of the $x_{j}^{l}$ 's $(l=1,2)$ with respect to the basis $Y\left\{u_{i}^{1}: 1 \leq i \leq k\right\}\left\{u_{i}^{2}: 1 \leq i \leq k\right\}$ in the case $l=2$ and $j=i_{0}$ only. By (S1) we know that $N\left(\left\langle Y X_{1}^{+} X_{2}^{+} X_{2}^{-}\right\rangle\right)$is freely generated by some $\Phi_{1}^{2}, \ldots, \Phi_{r}^{2}$ over $N\left(\left\langle Y X_{1}^{+}\right\rangle\right)$that are of the following form:

$$
\sum_{1 \leq s<t \leq k} \alpha_{s t}^{j}\left(\left(x_{s}^{2} \wedge x_{t}^{2}\right)-\left(x_{s}^{1} \wedge x_{t}^{1}\right)\right)+\sum_{1 \leq t \leq k}\left(y_{t}^{j} \wedge\left(x_{t}^{2}-x_{t}^{1}\right)\right)+\sum_{k<t \leq r}\left(y_{t}^{j} \wedge z_{t}^{2}\right)
$$

For every $t$ with $1 \leq t \leq k$ there exist $s$ and $j$ such that $s<t$ and $\alpha_{s t}^{j} \neq 0$ or $t<s$ and $\alpha_{t s}^{j} \neq 0$. Choose $l$ and $j$ such that $l<i_{0}$ and $\alpha_{l i_{0}}^{j} \neq 0$ or $i_{0}<l$ and $\alpha_{i_{0} l}^{j} \neq 0$. If $\Phi_{j}^{2}$ is rewritten with respect to $Y\left\{u_{i}^{1}: 1 \leq i \leq k\right\}\left\{u_{i}^{2}: 1 \leq i \leq k\right\}$, then $u_{l}^{2} \wedge u_{i_{0}}^{2}$ or $u_{i_{0}}^{2} \wedge u_{l}^{2}$ respectively occurs exactly once in this representation of $\Phi_{j}^{2}$. (5) is used. But this contradicts Lemma 4.3, if we consider a suitable basis of $D$ that is consistent with $Y\left\{u_{1}^{1}, \ldots, u_{k}^{1}\right\}\left\{u_{1}^{2}, \ldots, u_{k}^{2}\right\}$.

It remains to show that $S_{1}^{+e}=\emptyset$. Then $S_{1}^{+e}=\emptyset$ and $Z_{2}^{+e}=\emptyset$ imply $S_{2}^{+e}=\emptyset$. Let us assume that $S_{1}^{+e} \neq \emptyset$. For $u_{i_{0}}^{1} \in S_{1}^{+e}$ we have $u_{i_{0}}^{1}=x_{i_{0}}^{1}$. If $u_{i_{0}}^{1}$ is not involved in the representation of any $w_{j}^{+}$with respect to $Y^{c} Y^{d} S_{1}^{+e}$, then we obtain a contradiction as above:

We rewrite $\Phi_{1}^{2}, \ldots, \Phi_{r}^{2}$ in (S1) with respect to $Y\left\{u_{1}^{1}, \ldots, u_{k}^{1}\right\}\left\{u_{1}^{2}, \ldots, u_{k}^{2}\right\}$. Then there is some $\Phi_{j}^{2}$ and some $t$ such that $\Phi_{j}^{2}$ contains exactly once the basic commutator $u_{i_{0}}^{1} \wedge u_{t}^{1}$ or $u_{t}^{1} \wedge u_{i_{0}}^{1}$ respectively. Again this is impossible by Lemma 4.3.

Therefore we assume now that $u_{i_{0}}^{1}$ occurs in some $w_{m}^{+}$. For all $i$ with $k_{0}<i \leq k$ we define $w_{i}^{+}=q_{i} u_{i_{0}}+w_{i}$, where $w_{i} \in\left\langle Y^{c} Y^{d}\left(S_{1}^{+e} \backslash\left\{u_{i_{0}}^{1}\right\}\right)\right\rangle$. By assumption $q_{m} \neq 0$. Then $k_{0}<m$. We want to procduce a situation such that $q_{i} \neq 0$ for exactly one $i$. Otherwise we fix $m$ minimal with $q_{m} \neq 0$. We need $m \neq i_{0}$. If $i_{0} \leq k_{0}$, then this is true. If $k_{0}<i_{0}$, then $k_{1}<i_{0}$ by (3). In the case $k_{1}<i_{0}=m$ we choose another $m_{1}$ with $q_{m_{1}} \neq 0$. Then $m<m_{1}$. We can exchange $x_{m}^{j}$ and $x_{m_{1}}^{j}$ by (S4). By construction (1), (2), (3), (4), and (5) remain true. Therefore we can assume w.l.o.g. that $m \neq i_{0}$ and $m$ is minimal. Now we use (S4) again and apply the 
following well-tolerated automorphism determined by

$$
f\left(x_{i}^{j}\right)= \begin{cases}x_{i}^{j}-\left(q_{i} / q_{m}\right) x_{m}^{j} & \text { if } m<i \text { and } x_{i}^{2}=u_{i}^{2}+q_{i} u_{i_{0}}^{1}+w_{i}, \\ x_{i}^{j} & \text { otherwise }\end{cases}
$$

In the first case of the definition we have

$$
\begin{aligned}
f\left(x_{i}^{2}\right) & =u_{i}^{2}+q_{i} u_{i_{0}}^{1}+w_{i}-\left(q_{i} / q_{m}\right)\left(u_{m}^{2}+q_{m} u_{i_{0}}^{1}+w_{m}\right) \\
& =\left(u_{i}^{2}-\left(q_{i} / q_{m}\right) u_{m}^{2}\right)+\left(w_{i}-\left(q_{i} / q_{m}\right) w_{m}\right)
\end{aligned}
$$

and $f\left(x_{i}^{1}\right)=x_{i}^{1}-\left(q_{i} / q_{m}\right) x_{m}^{1}$.

Note that $u_{i}^{1}=x_{i}^{1}$ for $u_{i}^{1} \in S_{1}^{+e}$; in particular, $u_{i_{0}}^{1}=x_{i_{0}}^{1}$. Since $m \neq i_{0}$ we have for $k_{0} \leq i \leq m$

$$
w_{i} \in\left\langle Y^{c} Y^{d} f\left(S_{1}^{+e}\right) \backslash\left\{f\left(x_{i_{0}}^{1}\right)\right\}\right\rangle
$$

and for $m<i$

$$
w_{i}-\left(q_{i} / q_{m}\right) w_{m} \in\left\langle Y^{e} Y^{d} f\left(S_{1}^{+e} \backslash\left\{f\left(x_{i_{0}}^{1}\right)\right\}\right\rangle .\right.
$$

Hence after this application of (S4) we can assume that all our assumptions remain true and that $x_{i_{0}}^{1}=u_{i_{0}}^{1}$ occurs in $w_{m}^{+}=q_{m} u_{i_{0}}^{1}+w_{m}$ where $q_{m} \neq 0$ but in no other $w_{i}^{+}$with respect to the basis $Y^{c} Y^{d} S_{1}^{+e}$. Again we rewrite $(\mathrm{S} 1)$ with respect to the basis

$$
Y\left\{u_{1}^{1}, \ldots, u_{k}^{1}\right\}\left\{u_{1}^{2}, \ldots, u_{k}^{2}\right\}
$$

Then there is some $\Phi_{j}^{2}$ in $N\left(\left\langle Y X_{1}^{+} X_{2}^{+} X_{2}^{-}\right\rangle\right)$and some $t$ such that $\alpha_{m t}^{j} \neq 0$ or $\alpha_{t m}^{j} \neq 0$, and $u_{i_{0}}^{1} \wedge u_{t}^{2}$ or $u_{t}^{2} \wedge u_{i_{0}}^{1}$ occurs in the representation of (S1) exactly once. Again we have a contradiction to Lemma 4.3.

To prove (2) we apply (S4) similarly as above and obtain:

(6) $X_{2}^{+}=S_{2}^{+c x} S_{2}^{+e}$, which means $\left\{x_{1}^{2}, \ldots, x_{k_{0}}^{2}\right\}=S_{2}^{+c x}$ and $\left\{x_{k_{0}+1}^{2}, \ldots, x_{k}^{2}\right\}=$ $S_{2}^{+e}$.

(7) For $1 \leq i \leq k_{0}$ either $x_{i}^{1}=u_{i}^{1} \in S_{1}^{+e}$, or there exists $y_{i} \in\langle Y\rangle$ with $u_{i}^{1}=$ $x_{i}^{1}-y_{i} \in S_{1}^{+b x}$.

(8) There exists $k_{1}$ such that $k_{0} \leq k_{1} \leq k$,

$$
\left\{u_{i}^{1}: 1 \leq i \leq k_{0}, u_{i}^{1} \in B\right\}\left\{x_{k_{0}+1}^{1}, \ldots, x_{k_{1}}^{1}\right\}
$$

can be considered as $S_{1}^{+b x}$, and

$$
\left\{u_{i}^{1}: 1 \leq i \leq k_{0}, u_{i}^{1} \notin B\right\}\left\{x_{k_{1}+1}^{1}, \ldots, x_{k}^{1}\right\}
$$

can be considered as $S_{1}^{+e}$.

We define $u_{i}^{1}=x_{i}^{1}$ for $k_{0}<i \leq k$. Since $Y S_{1}^{+b x} S_{1}^{+e} Z_{2}^{+c x} Z_{2}^{+e}$ is a suitable basis for $\left\langle Y X_{1}^{+} X_{2}^{+}\right\rangle$, we have:

(9) $Y S_{1}^{+b x} S_{1}^{+e}\left\{x_{1}^{2}, \ldots, x_{k_{0}}^{2}\right\}$ is the initial part of a suitable basis of $\left\langle Y X_{1}^{+} X_{2}^{+}\right\rangle$.

We define $u_{i}^{2}=x_{i}^{2}$ for $1 \leq i \leq k_{0}$. As in the proof of 1 ) we get w.l.o.g.:

(10) For $k_{0}<i$

(a) either $u_{i}^{2}=x_{i}^{2}$ is not in $C$ modulo $\left\langle Y S_{1}^{+b x} S_{1}^{+e}\left\{u_{j}^{2}: j<i\right\}\right\rangle$,

(b) or $u_{i}^{2}=x_{i}^{2}-w_{i}^{+}$is in $C$ for some $w_{i}^{+} \in\left\langle Y^{b} Y^{d} S_{1}^{+b x} S_{1}^{+e}\right\rangle$. 
In the first case $u_{i}^{2}$ is in $Z_{2}^{+e}$ and in the second case $u_{i}^{2}$ is in $Z^{+c x}$. First we show that $Z_{2}^{+e}=\emptyset$. If $u_{i}^{2} \in Z_{2}^{+e}$, then we know by (10) that it does not occur in the representation of any $w_{j}^{+}$. Therefore we obtain a contradiction as in the proof of 1 . The next step is to show that $S_{1}^{+e}=\emptyset$. As above we assume that some $u_{i_{0}}^{1} \in S_{1}^{+e}$. Then $u_{i_{0}}^{1}=x_{i_{0}}^{1}$. If $u_{i_{0}}^{1}$ is not in the representation of some $w_{j}^{+}$, then we obtain a contradiction as above. Otherwise we show that we can assume w.l.o.g. that $u_{i_{0}}$ occurs exactly once in some $w_{j}^{+}$and get a contradiction.

It remains to show that $S_{2}^{+e}=\emptyset$. If $S_{2}^{+e} \neq \emptyset$, then by (6) and $S_{1}^{+e}=\emptyset$ we have $k_{0}<k_{1}=k$, and $x_{i}^{2} \in S_{2}^{+e}$ for all $i>k_{0}$. Then $x_{i}^{2}=u_{i}^{2}+w_{i}^{+}$, where $u_{i}^{2} \in C$ and $w_{i}^{+} \in\left\langle Y^{b} Y^{d} S_{1}^{+b x}\right\rangle$ as in (10b) for $i>k_{0} . w_{i}^{+}$has to contain some $u_{l}^{1}$ of $S_{1}^{+b x}$, since $Z_{2}^{+e}=\emptyset$. As in the proof of (1) we can show that w.l.o.g. some $u_{l}^{1}$ occurs in $w_{k_{0}+1}^{+}$ but not in any $w_{i}^{+}$with $i>k_{0}+1$. Again we get a contradiction to Lemma 4.3, since some $\Phi_{j}^{2}$ in (S1) has to contain a basic commutator $u_{t}^{2} \wedge u_{l}^{1}$ or $u_{l}^{1} \wedge u_{t}^{2}$ exactly once, where $u_{t}^{2} \in Z_{2}^{c x}$ and $u_{l}^{1} \in S_{1}^{+b x}$, a contradiction.

Now all our efforts are concentrated on the proof of the following:

Lemma 6.4. Assume that $A, B$, and $C$ are finite structures in $\mathcal{S}$ that satisfy $(\Sigma 3)$ and $(\Sigma 4)^{m}$. Let $A$ be a common substructure of $B$ and $C, B$ a minimal extension of $A$ that is not realized in $C$, and let $A$ be n-selfsufficient in $C$, where $2 m^{2}, \operatorname{dim}(B / A)+2 \leq n$. Let $D$ be $B \star_{A} C$. Then $C$ is selfsufficient in $D, B$ is $n$-selfsufficient in $D$, and $D$ satisfies $(\Sigma 3)$ and $(\Sigma 4)^{m}$.

By Theorem 4.1 it remains to show $(\Sigma 4)^{m}$ for $D$. Before we start we need three further lemmas.

Lemma 6.5. Assume the situation of Lemma 6.4. Let $Y X_{1}^{+} X_{2}^{+} X_{2}^{-} \ldots X_{h}^{+} X_{h}^{-}$be a reduced $k$-sequence with $r,|Y|+k<m$. For $2 \leq j \leq h$ we suppose that neither $\left\langle Y X_{1}^{+} X_{j}^{+} X_{j}^{-}\right\rangle \cap C=\left\langle Y X_{1}^{+}\right\rangle \cap C$ nor $\left\langle Y X_{1}^{+} X_{j}^{+} X_{j}^{-}\right\rangle \cap B=\left\langle Y X_{1}^{+}\right\rangle \cap B$ occurs. Then $h \leq 2(\max \{|Y|+k, r\})+1$.

Proof. Let $Q=Q^{a} Q^{b} Q^{c} Q^{d}$ be a suitable basis of $\left\langle Y X_{1}^{+}\right\rangle$that respects $\langle Y\rangle$. Assume that $S_{j}^{a} S_{j}^{b y} S_{j}^{b x} S_{j}^{c x} S_{j}^{e}$ is a suitable extension of $Q$ for $\left\langle Q X_{j}^{+} X_{j}^{-}\right\rangle$. By the assumptions of the lemma at least one of the following conditions is fulfilled:

(a) $S_{j}^{b x} S_{j}^{c x} S_{j}^{e}=\emptyset$.

(b) $S_{j}^{b x} S_{j}^{e} \neq \emptyset$ and $S_{j}^{a} S_{j}^{b y} S_{j}^{c x} \neq \emptyset$.

(c) $S_{j}^{c x} S_{j}^{e} \neq \emptyset$ and $S_{j}^{a} S_{j}^{b y} S_{j}^{b x} \neq \emptyset$.

Let $K$ be an extension of $H$. In the previous section, before Lemma 5.1, we described how to find a suitable basis of $K$, respecting a given suitable basis of $H$. $Q=Q^{a} Q^{b} Q^{c} Q^{d}$ is a suitable basis of $\left\langle Y X_{1}^{+}\right\rangle$that respects $\langle Y\rangle$. That means the following by definition:

$$
Q^{a}=Y^{a} Z_{1}^{a}, \quad Q^{b}=Y^{b} Z_{1}^{b y} Z_{1}^{b x}, \quad Q^{c}=Y^{c} Z_{1}^{c y} Z_{1}^{c x}, \quad Q^{d}=Y^{e 1} Z_{1}^{e},
$$

where $Y^{a} Y^{b} Y^{c} Y^{d}$ with $Y^{d}=Y^{e 1}\left\{u+v \in\langle Y\rangle: u \in Z_{1}^{b y}, v \in Z_{1}^{c y}\right\}$ is a suitable basis of $\langle Y\rangle$. This is the first step in an induction on $j \leq h$. For $1 \leq j \leq h$ we construct $Z_{j}=Z_{j}^{a} Z_{j}^{b y} Z_{j}^{b x} Z_{j}^{c x} Z_{j}^{e}$ and $Z_{j}^{c y}, Y^{e j}, Z_{1}^{e j}, \ldots, Z_{j-1}^{e j}, Z_{j}^{e j}=Z_{j}^{e}$ such that:

- For every $u \in Z_{j}^{b y}$ there is exactly one $v \in Z_{j}^{c y}$, and conversely for every $v \in Z_{j}^{c y}$ there is exactly one $u \in Z_{j}^{b y}$, such that

$$
u+v \in\left\langle Y X_{1}^{+} X_{2}^{+} X_{2}^{-} \ldots X_{j-1}^{+} X_{j-1}^{-}\right\rangle .
$$


- $\langle Y\rangle$ has the suitable basis

$$
Y^{a} Y^{b} Y^{c} Y^{e j}\left\{u+v \in\langle Y\rangle: u \in Z_{k}^{b y}, v \in Z_{k}^{c y}, 1 \leq k \leq j\right\}
$$

where $Y^{a}$ is a basis of $\langle Y\rangle \cap A, Y^{a} Y^{b}$ is a basis of $\langle Y\rangle \cap B, Y^{a} Y^{c}$ is a basis of $\langle Y\rangle \cap C$, and

$$
Y^{e j}\left\{u+v \in\langle Y\rangle: u \in Z_{k}^{b y}, v \in Z_{k}^{b c}, 1 \leq k \leq j\right\}
$$

is the part that is linearly independent over $(\langle Y\rangle \cap B)+(\langle Y\rangle \cap C)$.

- $\left\langle Y X_{1}^{+}\right\rangle$has the suitable basis

$$
\begin{aligned}
Y^{a} Z_{1}^{a} Y^{b} Z_{1}^{b y} Z_{1}^{b x} Y^{c} Z_{1}^{c y} Z_{1}^{c x} Y^{e j} Z_{1}^{e j}\{u & +v \in\left\langle Y X_{1}^{+}\right\rangle: \\
u & \left.\in Z_{k}^{b y}, v \in Z_{k}^{c y}, 2 \leq k \leq j\right\},
\end{aligned}
$$

where $Y^{a} Z_{1}^{a}$ is a basis of $\left\langle Y X_{1}^{+}\right\rangle \cap A, Y^{a} Z_{1}^{a} Y^{b} Z_{1}^{b y} Z_{1}^{b x}$ is a basis of $\left\langle Y X_{1}^{+}\right\rangle \cap B$, $Y^{a} Z_{1}^{a} Y^{c} Z_{1}^{c y} Z_{1}^{c x}$ is a basis of $\left\langle Y X_{1}^{+}\right\rangle \cap C$, and

$$
Y^{e j} Z_{1}^{e j}\left\{u+v \in\left\langle Y X_{1}^{+}\right\rangle: u \in Z_{k}^{b y}, v \in Z_{k}^{c y}, 2 \leq k \leq j\right\}
$$

is a basis of $\left\langle Y X_{1}^{+}\right\rangle$over $\left(\left\langle Y X_{1}^{+}\right\rangle \cap B\right)+\left(\left\langle Y X_{1}^{+}\right\rangle \cap C\right)$.

- For $\left\langle Y X_{1}^{+} X_{2}^{+} X_{2}^{-} \ldots X_{i}^{+} X_{i}^{-}\right\rangle$with $1<i \leq j$ we obtain a basis

$$
\begin{gathered}
Y^{a} Z_{1}^{a} Z_{2}^{a} \ldots Z_{i}^{a}, Y^{b} Z_{1}^{b y} Z_{1}^{b x} Z_{2}^{b y} Z_{2}^{b x} \ldots Z_{i}^{b y} Z_{i}^{b x}, Y^{c} Z_{1}^{c y} Z_{1}^{c x} Z_{2}^{c y} Z_{2}^{c x} \ldots Z_{i}^{c y} Z_{i}^{c x}, \\
Y^{e j} Z_{1}^{e j} Z_{2}^{e j} \ldots Z_{i}^{e j}\left\{u+v \in\left\langle Y X_{1}^{+} X_{2}^{+} X_{2}^{-} \ldots X_{i}^{+} X_{i}^{-}\right\rangle:\right. \\
\left.u \in Z_{k}^{b y}, v \in Z_{k}^{c y}, i<k \leq j\right\},
\end{gathered}
$$

where the $a$-part is a basis for

$$
\left\langle Y X_{1}^{+} X_{2}^{+} X_{2}^{-} \ldots X_{i}^{+} X_{i}^{-}\right\rangle \cap A
$$

the union of the $a$-part and the $b$-part is a basis for

$$
\left\langle Y X_{1}^{+} X_{2}^{+} X_{2}^{-} \ldots X_{i}^{+} X_{i}^{-}\right\rangle \cap B
$$

the union of the $a$-part and the $c$-part is a basis for

$$
\left\langle Y X_{1}^{+} X_{2}^{+} X_{2}^{-} \ldots X_{i}^{+} X_{i}^{-}\right\rangle \cap C
$$

and the remaining part is a basis of

$$
\begin{gathered}
\left\langle Y X_{1}^{+} X_{2}^{+} X_{2}^{-} \ldots X_{i}^{+} X_{i}^{-}\right\rangle \\
\text {over }\left(\left\langle Y X_{1}^{+} X_{2}^{+} X_{2}^{-} \ldots X_{i}^{+} X_{i}^{-}\right\rangle \cap B\right)+\left(\left\langle Y X_{1}^{+} X_{2}^{+} X_{2}^{-} \ldots X_{i}^{+} X_{i}^{-}\right\rangle \cap C\right) .
\end{gathered}
$$

The $Z_{j}$ are constructed by induction on $j$. Assume $Z_{j}$ has been built; we start the construction of $Z_{j+1}$. $Z_{j+1}^{a}$ is obtained as usual. Then $Z_{j+1}^{b y}=\bigcup_{0 \leq i \leq j} Z_{j+1}^{b y}(i)$ and $Z_{j+1}^{c y}=\bigcup_{0 \leq i \leq j} Z_{j+1}^{c y}(i)$, where $Z_{j+1}^{b y}(i)$ and $Z_{j+1}^{c y}(i)$ are obtained by induction on $i$. Let $Z_{j+1}^{b y}(0)$ be a basis of $(\langle Y\rangle+C) \cap B \cap\left\langle Y X_{1}^{+} X_{2}^{+} X_{2}^{-} \ldots X_{j+1}^{+} X_{j+1}^{-}\right\rangle$over $\left(B \cap\left\langle Y X_{1}^{+} X_{2}^{+} X_{2}^{-} \ldots X_{j}^{+} X_{j}^{-}\right\rangle\right)+\left\langle Z_{j+1}^{a}\right\rangle$. To get $Z_{j+1}^{c y}(0)$, choose for every $u \in$ $Z_{j+1}^{b y}(0)$ some $v$ in $C$ such that $u+v \in\langle Y\rangle$. If $Z_{j+1}^{b y}(i)$ and $Z_{j+1}^{c y}(i)$ are constructed, then define $Z_{j+1}^{b y}(i+1)$ to be a basis of $\left(\left\langle Y X_{1}^{+} X_{2}^{+} X_{2}^{-} \ldots X_{i+1}^{+} X_{i+1}^{-}\right\rangle+C\right) \cap B \cap$ $\left\langle Y X_{1}^{+} X_{2}^{+} X_{2}^{-} \ldots X_{j+1}^{+} X_{j+1}^{-}\right\rangle$over

$$
\left(B \cap\left\langle Y X_{1}^{+} X_{2}^{+} X_{2}^{-} \ldots X_{j}^{+} X_{j}^{-}\right\rangle\right)+\left\langle Z_{j+1}^{a} Z_{j+1}^{b y}(0) \ldots Z_{j+1}^{b y}(i)\right\rangle
$$


To obtain $Z_{j+1}^{c y}(i+1)$ we choose for every $u \in Z_{j+1}^{b y}(i+1)$ some $v \in C$ such that $u+v \in\left\langle Y X_{1}^{+} X_{2}^{+} X_{2}^{-} \ldots X_{i+1}^{+} X_{i+1}^{-}\right\rangle$. Then $Z_{j+1}^{b x}$ and $Z_{j+1}^{c x}$ are obtained as usual. $Y^{e(j+1)}$ is a basis of $\langle Y\rangle$ over

$$
\left(\left\langle Y X_{1}^{+} X_{2}^{+} X_{2}^{-} \ldots X_{j+1}^{+} X_{j+1}^{-}\right\rangle \cap B\right)+\left(\left\langle Y X_{1}^{+} X_{2}^{+} X_{2}^{-} \ldots X_{j+1}^{+} X_{j+1}^{-}\right\rangle \cap C\right)
$$

and, for $1 \leq i \leq j+1, Z_{i}^{e(j+1)}$ is a basis of $\left\langle Y X_{1}^{+} X_{2}^{+} X_{2}^{-} \ldots X_{i}^{+} X_{i}^{-}\right\rangle$over

$$
\begin{aligned}
& \left(\left\langle Y X_{1}^{+} X_{2}^{+} X_{2}^{-} \ldots X_{j+1}^{+} X_{j+1}^{-}\right\rangle \cap B\right) \\
& \quad+\left(\left\langle Y X_{1}^{+} X_{2}^{+} X_{2}^{-} \ldots X_{j+1}^{+} X_{j+1}^{-}\right\rangle \cap C\right)+\left\langle Y_{j+1}^{e} Z_{j+1}^{e 1} \ldots Z_{i-1}^{e(j+1)}\right\rangle .
\end{aligned}
$$

Now after modifying $Y^{d}$ and all $Z_{j}^{e}$ we can assume:

$$
Y^{d}=Y^{e h}\left\{u+v \in\langle Y\rangle: u \in Z_{l}^{b y}, v \in Z_{l}^{c y} \quad \text { for some } l \text { with } 1 \leq l \leq h\right\}
$$

and

$$
Z_{j}^{e}=Z_{j}^{e h}\left\{u+v \in\left\langle Z_{j}^{e}\right\rangle: u \in Z_{l}^{b y}, v \in Z_{l}^{c y} \quad \text { for some } l \text { with } \quad j<l \leq h\right\}
$$

for $1 \leq j \leq h$. We say:

$Y Z_{1} \ldots Z_{h}$ gives us a suitable basis of $\left\langle Y X_{1}^{+} X_{2}^{+} X_{2}^{-} \ldots X_{h}^{+} X_{h}^{-}\right\rangle$

$$
\text { that respects }\langle Y\rangle,\left\langle Y X_{1}^{+}\right\rangle, \ldots,\left\langle Y X_{1}^{+} X_{2}^{+} X_{2}^{-} \ldots X_{h-1}^{+} X_{h-1}^{-}\right\rangle \text {. }
$$

If $Z_{j}=Z_{j}^{a} Z_{j}^{b y}$, then we can carry out the procedure above so that we have $Z_{j}^{+}=Z_{j}^{+a} Z_{j}^{+b y}$ as a suitable extension for

$$
\left\langle Y X_{1}^{+} X_{2}^{+} X_{2}^{-} \ldots X_{j-1}^{+} X_{j-1}^{-} X_{j}^{+}\right\rangle
$$

over $\left\langle Y X_{1}^{+} X_{2}^{+} X_{2}^{-} \ldots X_{j-1}^{+} X_{j-1}^{-}\right\rangle$and $Z_{j}^{-}=Z_{j}^{-a} Z^{-b y}$ as a suitable extension for

$$
\left\langle Y X_{1}^{+} X_{2}^{+} X_{2}^{-} \ldots X_{j}^{+} X_{j}^{-}\right\rangle
$$

over $\left\langle Y X_{1}^{+} X_{2}^{+} X_{2}^{-} \ldots X_{j-1}^{+} X_{j-1}^{-} X_{j}^{+}\right\rangle$.

Note that $Q^{d}=Y^{d} Z_{1}^{e}$. We suppose that $Q^{d}=\left\{u_{1}+v_{1}, \ldots, u_{l_{1}}+v_{l_{1}}\right\}$. By our assumption either $u_{i}+v_{i}$ is not in

$$
\left(B \cap\left\langle Y X_{1}^{+} X_{2}^{+} X_{2}^{-} \ldots X_{h}^{+} X_{h}^{-}\right\rangle\right)+\left(C \cap\left\langle Y X_{1}^{+} X_{2}^{+} X_{2}^{-} \ldots X_{h}^{+} X_{h}^{-}\right\rangle\right)
$$

or $u_{i} \in Z_{j}^{b y}$ and $v_{i} \in Z_{j}^{c y}$ for some $j$ with $2 \leq j \leq h$. Define $U_{1}=Q^{b}\left\{u_{1}, \ldots, u_{l_{1}}\right\}$, $V_{1}=Q^{c}\left\{v_{1}, \ldots, v_{l_{1}}\right\}$, and $W_{1}=Q^{a}$.

If $U_{j-1}, V_{j-1}$, and $W_{j-1}$ are defined and $Z_{j}=Z_{j}^{a} Z_{j}^{b y} Z_{j}^{b x} Z_{j}^{c x} Z_{j}^{e}$, where $Z_{j}^{e}=$ $\left\{u_{l_{j-1}+1}+v_{l_{j-1}+1}, \ldots, u_{l_{j}}+v_{l_{j}}\right\}$, then we define

$U_{j}=U_{j-1} Z^{b x}\left\{u_{l_{j-1}+1}, \ldots, u_{l_{j}}\right\}$,

$V_{j}=V_{j-1} Z^{c x}\left\{v_{l_{j-1}+1}, \ldots, v_{l_{j}}\right\}$, and

$W_{j}=W_{j-1} Z_{j}^{a}$.

By assumption $u_{i}+v_{i} \in Z_{j}^{e}$ is either not in

$$
\left(B \cap\left\langle Y X_{1}^{+} X_{2}^{+} X_{2}^{-} \ldots X_{h}^{+} X_{h}^{-}\right\rangle\right)+\left(C \cap\left\langle Y X_{1}^{+} X_{2}^{+} X_{2}^{-} \ldots X_{h}^{+} X_{h}^{-}\right\rangle\right)
$$

or $u_{i} \in Z_{l}^{b y}$ and $v_{i} \in Z_{l}^{c y}$ for some $l$ with $j<l \leq h$.

Now the fact that (a), (b), or (c) is true implies that one of the following conditions is fulfilled:

I. $Z_{j}^{b x} Z_{j}^{c x} Z_{j}^{e}=\emptyset$.

II. $Z_{j}^{b x} Z_{j}^{e} \neq \emptyset$ and $Z_{j}^{a} Z_{j}^{b y} Z_{j}^{c x} \neq \emptyset$.

III. $Z_{j}^{c x} Z_{j}^{e} \neq \emptyset$ and $Z_{j}^{a} Z_{j}^{b y} Z_{j}^{b x} \neq \emptyset$. 
First we assume that $Y=Y^{a} \subseteq A$ and there is some $X_{j}^{+} \subseteq A$. By (S3) w.l.o.g. $X_{1}^{+} \subseteq A$. The case $X_{1}^{+}=\emptyset(k=0)$ is included. Then $Q=Q^{a} \subseteq A$. By (S1) a suitable basis of $\left\langle Y X_{1}^{+} X_{j}^{+} X_{j}^{-}\right\rangle$cannot be of the form $Q S_{j}^{e}$ for any $j$. If $k=0$, use Lemma 4.4. Hence $\left(\left\langle Y X_{1}^{+} X_{j}^{+} X_{j}^{-}\right\rangle \cap B\right)+\left(\left\langle Y X_{1}^{+} X_{j}^{+} X_{j}^{-}\right\rangle \cap C\right) \neq\left\langle Y X_{1}^{+}\right\rangle$for all $j$. Then Lemma 5.2 implies that either all $X_{j}^{+} X_{j}^{-}$are in $B$ or they are all in $C$. $(\Sigma 4)^{m}$ for $B$ and $C$ gives the assertion. In all other cases we prove the following claim (11) that implies the assertion, as we will explain.

(11) There are functions $f$ and $g$ with $f(1)=g(1)=0$ and $f(i-1) \leq f(i)$, $g(i-1) \leq g(i)$ for $2 \leq i \leq h$ and relations $\Delta_{1}, \ldots, \Delta_{f(j)} \in N(B)$ and $\Theta_{1}, \ldots, \Theta_{g(j)} \in N(C)$ such that for $2 \leq j \leq h$ :

(i) If we have Case II, then $f(j)=f(j-1)+\left|U_{j} \backslash U_{j-1}\right|+1$.

(ii) If we have Case III, then $g(j)=g(j-1)+\left|V_{j} \backslash V_{j-1}\right|+1$.

(iii) If we have Case I, then $f(j-1) \leq f(j) \leq f(j-1)+1, g(j-1) \leq g(j) \leq$ $g(j-1)+1$, and $f(j)=f(j-1)+1$ or $g(j)=g(j-1)+1$.

(iv) $\Delta_{1}, \ldots, \Delta_{f(j)}$ are linearly independent over $\wedge^{2} A$.

(v) $\Theta_{1}, \ldots, \Theta_{g(j)}$ are linearly independent over $\wedge^{2} A$.

(vi) If $f(j-1)<i \leq f(j)$, then $\Delta_{i}$ is in $\left(\wedge^{2}\left\langle U_{j} W_{j}\right\rangle+\wedge^{2} A\right) \cap N(B)$. If $\Delta_{i}$ is written as a linear combination of basic commutators with respect to a suitable basis of $D$ that extends $U_{j} W_{j}$, then every $u_{l}$ that is not in $\bigcup_{2 \leq s \leq j} Z_{s}^{b y}$ occurs in basic commutators of the form $a \wedge u$ only, where $a \in A$.

(vii) If $g(j-1)<i \leq g(j)$, then $\Theta_{i}$ is in $\left(\wedge^{2}\left\langle V_{j} W_{j}\right\rangle+\wedge^{2} A\right) \cap N(C)$.If $\Theta_{i}$ is written as a linear combination of basic commutators with respect to a suitable basis of $D$, that extends $V_{j} W_{j}$, then every $v_{l}$, that is not in $\cup_{2 \leq s \leq j} Z_{s}^{c y}$, occurs in basic commutators of the form $a \wedge v$ only, where $a \in A$.

First we show that (11.i-vii) implies $h \leq 2(k+|Y|)+1$. Otherwise w.l.o.g. $h=2(k+|Y|)+2$. By (11.i-iii) we have

$$
\left(f(h)-\left|U_{h} \backslash U_{1}\right|\right)+\left(g(h)-\left|V_{h} \backslash V_{1}\right|\right) \geq h-1>2(k+|Y|) .
$$

It follows that

$$
f(h)>k+|Y|+\left|U_{h}\right|-\left|U_{1}\right| \geq\left|U_{h}\right| \quad \text { or } \quad g(h)>k+|Y|+\left|V_{h}\right|-\left|V_{1}\right| \geq\left|V_{h}\right| .
$$

By (11.iv) or (11.v) respectively we have

$$
\operatorname{dim}\left(N\left(\left\langle U_{h} A\right\rangle\right)\right)-\operatorname{dim}(N(A))>\left|U_{h}\right|, \quad \text { or } \quad \operatorname{dim}\left(N\left(\left\langle V_{h} A\right\rangle\right)\right)-\operatorname{dim}(N(A))>\left|V_{h}\right| .
$$

This is equivalent to

$$
\delta\left(\left\langle U_{h} A\right\rangle\right)<\delta(A) \quad \text { or } \quad \delta\left(\left\langle V_{h} A\right\rangle\right)<\delta(A) .
$$

The first inequality contradicts the self-sufficiency of $A$ in $B$. Note that

$$
\left|V_{h}\right| \leq|Y|+\left|X_{1}^{+}\right|+(h-1)\left|X_{2}^{+} X_{2}^{-}\right|<2 m^{2},
$$

because $k+|Y|, r<m$. Therefore the second inequality contradicts the $2 m^{2}$ selfsufficiency of $A$ in $C$.

Now we assume that $Y \nsubseteq A$ or $Y \subseteq A$ but no $X_{j}^{+}$is in $A$. To prove (11) by induction on $j$ we suppose that (11) is true for $j-1$.

First we show (11) for $j$ in the Cases II and III. Let us assume Case II; Case III is similar. We have $Z_{j}^{b x} Z_{j}^{e} \neq \emptyset$ and $Z_{j}^{a} Z_{j}^{b y} Z_{j}^{c x} \neq \emptyset$. Then $f(j)=f(j-1)+\left|Z_{j}^{b x} Z_{j}^{e}\right|+1$. 
Since $\left\langle Q Z_{2} \ldots Z_{j}\right\rangle$ is a minimal extension of $\left\langle Q Z_{2} \ldots Z_{j-1}\right\rangle$, we obtain

$$
\delta\left(\left\langle Q Z_{2} \ldots Z_{j-1} Z_{j}^{a} Z_{j}^{b y} Z_{j}^{c x}\right\rangle\right)>\delta\left(\left\langle Q Z_{2} \ldots Z_{j-1}\right\rangle\right)=\delta\left(\left\langle Q Z_{2} \ldots Z_{j}\right\rangle\right) .
$$

Therefore

$$
\operatorname{dim}\left(N\left(\left\langle Q Z_{2} \ldots Z_{j}\right\rangle\right)\right)-\operatorname{dim}\left(N\left(\left\langle Q Z_{2} \ldots Z_{j-1} Z_{j}^{a} Z_{j}^{b y} Z_{j}^{c x}\right\rangle\right)\right)>\left|Z_{j}^{b x} Z_{j}^{e}\right|
$$

Denote $\left|Z_{j}^{b x} Z_{j}^{e}\right|+1=f(j)-f(j-1)$ by $s$. We choose relations $\Phi_{1}, \ldots, \Phi_{s}$ in $N\left(\left\langle Q Z_{2} \ldots Z_{j}\right\rangle\right)$ that are linearly independent over

$$
N\left(\left\langle Q Z_{2} \ldots Z_{j-1} Z_{j}^{a} Z_{j}^{b y} Z_{j}^{c x}\right\rangle\right) .
$$

This is equivalent to the following:

Every non-trivial linear combination of $\Phi_{1}, \ldots \Phi_{s}$ considered as

a linear combination of basic commutators over $Q Z_{2} \ldots Z_{j}$

contains a basic commutator with an element of $Z_{j}^{b x} Z_{j}^{e}$.

If we rewrite $\Phi_{i}$ as a linear combination of basic commutators with respect to $U_{j} V_{j} W_{j}$ then by Lemma $4.3 \Phi_{i}=\Phi_{i}^{b}+\Phi_{i}^{c}$, where $\Phi_{i}^{b} \in \wedge^{2}\left\langle W_{j} U_{j}\right\rangle, \Phi_{i}^{c} \in \wedge^{2}\left\langle W_{j} V_{j}\right\rangle$, and there is some $J_{i} \in \wedge^{2} A$ such that $\Phi_{i}^{b}+J_{i} \in N(B)$ and $\Phi_{i}^{c}-J_{i} \in N(C)$. We define $\Delta_{f(j-1)+i}=\Phi_{i}^{b}+J_{i}$. Then $\Delta_{f(j-1)+i} \in\left(\wedge^{2}\left\langle W_{j} U_{j}\right\rangle+\wedge^{2} A\right) \cap N(B)$. By construction every non-trivial combination of $\Delta_{f(j-1)+1}, \ldots, \Delta_{f(j)}$, represented as a linear combination of basic commutators over a suitable basis of $D$ that extends $W_{j} U_{j}$, contains a basic commutator with an element of $U_{j} \backslash U_{j-1}$. Therefore $\Delta_{1}, \ldots, \Delta_{f(j)}$ are linearly independent over $\wedge^{2} A$ by induction. It remains to show (11) (vi) for $\Delta_{f(j-1)+1}, \ldots, \Delta_{f(j)}$. This is an immediate consequence of Lemma 4.3 , if we use the suitable basis $Q Z_{2} \ldots Z_{j}$.

Now let us assume that $Y^{b} Y^{d} \neq \emptyset$. We show (11) for $j$ in the remaining Case I. We have $Z_{j}=Z_{j}^{a} Z_{j}^{b y}$. Take $y \in Y^{b} Y^{d}$. Define $y_{b}=y$, if $y \in Y^{b}$, and $y_{b} \in U_{1}$, $y_{c} \in V_{1}$, if $y=y_{b}+y_{c} \in Y^{d}$. By (S1) $N\left(\left\langle Y X_{1}^{+} X_{j}^{+} X_{j}^{-}\right\rangle\right)$is freely generated by $\Phi_{1}^{j} \ldots \Phi_{r}^{j}$, where $\Phi_{i}^{j}$ is of the form

$$
\sum_{1 \leq s<t \leq k} \alpha_{s t}^{i}\left(\left(x_{s}^{j} \wedge x_{t}^{j}\right)-\left(x_{s}^{1} \wedge x_{t}^{1}\right)\right)+\sum_{1 \leq t \leq k}\left(y_{t}^{i} \wedge\left(x_{t}^{j}-x_{t}^{1}\right)\right)+\sum_{k<t \leq r}\left(y_{t}^{i} \wedge z_{t}^{j}\right) .
$$

If we rewrite the $y_{t}^{i}$, s with respect to $Y$, then let $\gamma_{t}^{i}$ be the coefficient of $y \wedge\left(x_{t}^{j}-x_{t}^{1}\right)$ and $y \wedge z_{t}^{j}$ respectively in $\Phi_{i}^{j}$.

As mentioned above we have chosen $Z_{j}^{+}=Z_{j}^{+a} Z_{j}^{+b y}$ as a suitable extension for $\left\langle Y X_{1}^{+} X_{2}^{+} X_{2}^{-} \ldots X_{j-1}^{+} X_{j-1}^{-} X_{j}^{+}\right\rangle$over $\left\langle Y X_{1}^{+} X_{2}^{+} X_{2}^{-} \ldots X_{j-1}^{+} X_{j-1}^{-}\right\rangle$and $Z_{j}^{-}=$ $Z_{j}^{-a} Z_{j}^{-b y}$ for $\left\langle Y X_{1}^{+} X_{2}^{+} X_{2}^{-} \ldots X_{j}^{+} X_{j}^{-}\right\rangle$over $\left\langle Y X_{1}^{+} X_{2}^{+} X_{2}^{-} \ldots X_{j-1}^{+} X_{j-1}^{-} X_{j}^{+}\right\rangle$. Using an appropriate well-tolerated automorphism of $\left\langle Y X_{1}^{+} X_{2}^{+} X_{2}^{-} \ldots X_{j}^{+} X_{j}^{-}\right\rangle$, we may assume by (S4) that

$$
\begin{gathered}
\left\{x_{1}^{j}, \ldots, x_{k}^{j}\right\}=Z_{j}^{+} \text {modulo }\left\langle Y X_{1}^{+} X_{2}^{+} X_{2}^{-} \ldots X_{j-1}^{+} X_{j-1}^{-}\right\rangle, \\
\left\{z_{k+1}^{j}, \ldots, z_{r}^{j}\right\}=Z_{j}^{-} \text {modulo }\left\langle Y X_{1}^{+} X_{2}^{+} X_{2}^{-} \ldots X_{j-1}^{+} X_{j-1}^{-}\right\rangle .
\end{gathered}
$$

Let $x_{t}$ be the element of $Z_{j}^{+}$that corresponds to $x_{t}^{j}$, and let $z_{t}$ be the element of $Z_{j}^{-}$that corresponds to $z_{t}^{j}$. In the following we will find suitable $x_{t}=a \in Z_{j}^{+a}$ or $z_{t}=a \in Z_{j}^{-a}$ or $x_{t}=u \in Z^{+b y}$ or $z_{t}=u \in Z_{j}^{-b y}$ such that $y_{b} \wedge a$ or $y_{c} \wedge a$ or $y_{b} \wedge u$ 
or $y_{c} \wedge v$ has a non-zero coefficient in the representation of some $\Phi_{i}^{j}$ with respect to $W_{j} U_{j} V_{j}$, where $v \in Z_{j}^{c y}$ is obtained by $u+v \in\left\langle Y X_{1}^{+} X_{2}^{+} X_{2}^{-} \ldots X_{j-1}^{+} X_{j-1}^{-}\right\rangle$.

First we assume that we can choose $y=y_{b} \in Y^{b}$ or $y \in Y^{d}$ with $y=y_{b}+y_{c}$ where $y_{b} \in Z_{1}^{b y} Z_{2}^{b y} \ldots Z_{j-1}^{b y}$. Then $x_{t}^{j}=x_{t}+x_{t}^{0}+\epsilon_{t} y_{b}$ and $z_{t}^{j}=z_{t}+z_{t}^{0}$, where $x_{t}^{0}+\epsilon_{t} y_{b}$ and $z_{t}^{0}$ are in $\left\langle Q Z_{2} \ldots Z_{j-1}\right\rangle$ and $x_{t}^{0} \in\left\langle Q Z_{2} \ldots Z_{j-1} \backslash\left\{y_{b}\right\}\right\rangle$. In the representation of $\Phi_{i}^{j}$ with respect to $Q Z_{2} \ldots Z_{j}$ the coefficient $\eta_{t}^{i}$ of $y_{b} \wedge z_{t}$ is $\gamma_{t}^{i}$ and of $y_{b} \wedge x_{t}$ is $\gamma_{t}^{i}+\sum_{s<t} \alpha_{s t}^{i} \epsilon_{s}-\sum_{t<s} \alpha_{t s}^{i} \epsilon_{s}$. There has to be some $i$ such that at least one of these coefficients $\eta_{t}^{i}$ is not zero. Otherwise the well-tolerated automorphism $e$ defined by $e\left(x_{t}^{j}\right)=x_{t}^{j}-\epsilon_{t} y$ and $e\left(z_{t}^{j}\right)=z_{t}^{j}$ would give an (S1)-representation of the $\Phi_{i}^{j}$, s with respect to $(Y \backslash\{y\}) X_{1}^{+} X_{j}^{+} X_{j}^{-}$, a contradiction to (S4). Fix $i$ and $t$ with $\eta_{t}^{i} \neq 0$. If $x_{t}=a \in Z_{j}^{+a}$ for $t \leq k$ or $z_{t}=a \in Z_{j}^{-a}$ for $k<t$, then $\Phi_{i}^{j}$ contains $y_{b} \wedge a$ with the coefficient $\eta_{t}^{i}$ in its representation with respect to the basis $W_{j} U_{j} V_{j}$, because $a \notin W_{j-1} U_{j-1} V_{j-1}$. Otherwise $x_{t}=u \in Z_{j}^{+b y}$ for $t \leq k$ or $z_{t}=u \in Z_{j}^{-b y}$ for $k<t$. Note that $u \neq y_{b}$. Then there is some $v \in Z_{j}^{+c y}$ or $v \in Z_{j}^{-c y}$ such that $u+v \in Q^{d} Z_{2}^{e} \ldots Z_{j-1}^{e}$. In the representation of $\Phi_{i}^{j}$ with respect to $W_{j} U_{j} V_{j}$ the coefficient of $y_{b} \wedge u$ is $\left(\eta_{t}^{i}+\mu\right)$, where $\mu$ is the coefficient of $y_{b} \wedge v$ in this representation. Either $\eta_{t}^{i}+\mu \neq 0$ or $\mu \neq 0$. In the first case $y_{b} \wedge u$ occurs in this representation with the coefficient $\left(\eta_{t}^{i}+\mu\right) \neq 0$. In the second case $y_{b} \wedge v$ has the coefficient $\mu \neq 0$. This is impossible by Lemma 4.3 .

Now we suppose that $Y^{b}=\emptyset$ and there are no $l<j, y_{b} \in Z_{l}^{b y}$, and $y_{c} \in Z_{l}^{c y}$ such that $y_{b}+y_{c} \in Y^{d}$. By the construction of $Z_{1}, \ldots, Z_{h}$ we have that $Y^{d}$ is linearly independent over $\left.\left(\left\langle Q Z_{1} \ldots Z_{j-1}\right\rangle \cap B\right)+\left(\left\langle Q Z_{1} \ldots Z_{j-1}\right\rangle\right) \cap C\right)$. By assumption $Y^{d} \neq \emptyset$. As above, consider $x_{t}^{j}=x_{t}+x_{t}^{0}+\epsilon_{t} y, z_{t}^{j}=z_{t}+z_{t}^{0}$, where $x_{t}^{0}+\epsilon_{t} y, z_{t}^{0} \in$ $\left\langle Q Z_{2} \ldots Z_{j-1}\right\rangle$ and $x_{t}^{0} \in\left\langle Q Z_{2} \ldots Z_{j-1} \backslash\{y\}\right\rangle$. Again $\eta_{t}^{i}=\gamma_{t}^{i}$ is the coefficient of $y \wedge z_{t}$ and $\eta_{t}^{i}=\gamma_{t}^{i}+\sum_{s<t} \alpha_{s t}^{i} \epsilon_{s}-\sum_{t<s} \alpha_{t s}^{i} \epsilon_{s}$ is the coefficient of $y \wedge x_{t}$ for $t \leq k$ respectively in the representation of $\Phi_{i}^{j}$ with respect to the basis $Q Z_{2} \ldots Z_{j}$. By the same argument as above we obtain $i$ and $t$ with $\eta_{t}^{i} \neq 0$. We have to discuss the following cases:

(i) $Y^{d}$ is linearly independent over $\left(\left\langle Q Z_{1} \ldots Z_{j}\right\rangle \cap B\right)+\left(\left\langle Q Z_{1} \ldots Z_{j}\right\rangle \cap C\right)$.

(ii) There are some $y_{b} \in Z_{j}^{b y}$ and $y_{c} \in Z_{j}^{c y}$ such that $y=y_{b}+y_{c} \in Y^{d}$.

Again we have used the fact that $Y Z_{1} \ldots Z_{h}$ gives us a suitable basis of

$$
\left\langle Y X_{1}^{+} X_{2}^{+} X_{2}^{-} \ldots X_{h}^{+} X_{h}^{-}\right\rangle
$$

respecting

$$
\langle Y\rangle,\left\langle Y X_{1}^{+}\right\rangle, \ldots,\left\langle Y X_{1}^{+} X_{2}^{+} X_{2}^{-} \ldots X_{h-1}^{+} X_{h-1}^{-}\right\rangle .
$$

We consider Case (i) and choose any $y \in Y^{d}$. Then there is no $y_{b} \in Z_{j}^{b y}$ with $y-y_{b} \in Z_{j}^{c y}$. Let $\mu_{t}^{i}$ be the coefficient of $y \wedge z_{t}$ or $y \wedge x_{t}$ respectively in the representation of $\Phi_{i}^{j}$ with respect to the basis $W_{j} U_{j} V_{j}$. If $x_{t}=a \in Z_{j}^{+a}$ or $z_{t}=a \in Z_{j}^{-a}$ respectively, then $\mu_{t}^{i}=\eta_{t}^{i} \neq 0$ as above. Otherwise $x_{t}=u \in Z_{j}^{+b y}$ or $z_{t}=u \in Z_{j}^{-b y}$ respectively. Choose $v \in Z_{j}^{c y}$ such that $u+v \in Q^{d} Z_{2}^{e} \ldots Z_{j-1}^{e}$. By assumption $(u+v) \neq y$. Hence $\mu_{t}^{i}=\eta_{t}^{i}+\mu$, where $\mu$ is the coefficient of $y \wedge(u+v)$ in the representation of $\Phi_{i}^{j}$ with respect to the basis $Q Z_{2} \ldots Z_{j}$. If we consider the representation of $\Phi_{i}^{j}$ with respect to the basis $W_{j} U_{j} V_{j}$, then we have $\mu=0$ by Lemma 4.3. This case is not possible, since $\eta_{t}^{i} \neq 0$ would imply a contradiction. 
There remains Case (ii). We choose $y \in Y^{d}$ with some $y_{b} \in Z_{j}^{b y}$ such that $y-y_{b} \in Z_{j}^{c y}$. Assume $y_{b}=x_{t_{0}}$ or $y_{b}=z_{t_{0}}$ respectively. Then $\eta_{t_{0}}^{i}=0$ for all $i$ by Lemma 4.3. Note $i$ and $t$ are fixed above with $\eta_{t}^{i} \neq 0$. Therefore $x_{t}$ or $z_{t}$ respectively is different from $y_{b}$. If $x_{t}=a \in Z_{j}^{+a}$ or $z_{t}=a \in Z_{j}^{-a}$ respectively, then $\eta_{t}^{i} \neq 0$ is the coefficient of $y_{c} \wedge a$ in the representation of $\Phi_{i}^{j}$ with respect to the basis $W_{j} U_{j} V_{j}$. Otherwise $x_{t}=u \in Z_{j}^{+b y}$ or $z_{t}=u \in Z_{j}^{-b y}$ respectively. Choose $v \in Z_{j}^{c y}$ such that $u+v \in Q^{d} Z_{2}^{e} \ldots Z_{j-1}^{e}$. Let $\mu$ be the coefficient of $y \wedge(u+v)$ in the presentation of $\Phi_{i}^{j}$ with respect to $Q Z_{2} \ldots Z_{j}$. Define $\nu_{t}^{i}=0$ for $k<t, \nu_{t}^{i}=\alpha_{t_{0} t}^{i}$ for $t_{0}<t \leq k$, and $\nu_{t}^{i}=-\alpha_{t t_{0}}^{i}$ if $t<t_{0} \leq k$. We consider again the representation of $\Phi_{i}^{j}$ with respect to the basis $W_{j} U_{j} V_{j}$. Then

$$
\begin{aligned}
& \eta_{t}^{i}+\nu_{t}^{i}+\mu \text { is the coefficient of } y_{b} \wedge u ; \\
& \eta_{t}^{i}+\mu \text { is the coefficient of } y_{c} \wedge u ; \\
& \mu \text { is the coefficient of } y_{c} \wedge v .
\end{aligned}
$$

By Lemma 4.3, $\eta_{t}^{i}+\mu=0$. If $y_{b} \wedge u$ has the coefficient 0 , then $\nu_{t}^{i}=0$ and $\eta_{t}^{i} \neq 0$ implies $\mu \neq 0$. Hence either $y_{b} \wedge u$ or $y_{c} \wedge v$ has a non-zero coefficient.

Our assumption was that $Y^{b} Y^{d} \neq 0$. In all possible cases we have shown that there is some $\Phi_{i}^{j}$ that contains a basic commutator $y_{b} \wedge a$ or $y_{c} \wedge a$ or $y_{b} \wedge u$ or $y_{c} \wedge v$ in its representation with respect to $W_{j} U_{j} V_{j}$, where $a \in Z_{j}^{a}, u \in Z_{j}^{b y}$, and $v \in Z_{j}^{c y}$. Of course $y_{b} \wedge a$ cannot occur in the representation of any $\Delta_{i}$ with $i<f(j)$ as a linear combination of basic commutators with respect to $W_{j} U_{j} V_{j}$. The same is true for $y_{b} \wedge u$ and $\Delta_{i}$ with $i<f(j)$ and for $y_{c} \wedge v$ and $\Theta_{i}$ with $i<g(j)$, since the representation as a linear combination of basic commutators with respect to $W_{j} U_{j} V_{j}$ of $\Delta_{i}$ with $i<f(j)$ and of $\Theta_{i}$ with $i<g(j)$ contains $w \wedge u$ or $w \wedge v$ respectively for $w \in A$ only, by (11) (vi) and (vii). If we represent $\Phi_{i}^{j}$ as a linear combination of basic commutators with respect to a suitable basis of $D$ that contains $W_{j} U_{j} V_{j}$, then $\Phi_{i}^{j}=\Phi^{b}+\Phi^{c}$ and there is some $J \in \wedge^{2} A$ such that $\Phi^{b} \in \wedge^{2}\left\langle W_{j} U_{j}\right\rangle, \Phi^{c} \in \wedge^{2}\left\langle W_{j} V_{j}\right\rangle, \Phi^{b}+J \in N(B)$, and $\Phi^{c}-J \in N(C)$. We define $f(j)=f(j-1)+1$ and $\Delta_{f(j)}=\Phi^{b}+J$, if $y_{b} \wedge a$ or $y_{b} \wedge u$ occurs. Since $y_{b} \wedge a$ or $y_{b} \wedge u$ occurs in $\Delta_{l}$ for $1 \leq l \leq f(j)$ if and only if $l=f(j)$, we obtain the linear independence of $\Delta_{1}, \ldots, \Delta_{f(j)}$ by induction. Analogously we define $g(j)=g(j-1)+1$ and $\Theta_{g(j)}=\Phi^{c}-J$, if $y_{c} \wedge a$ or $y_{c} \wedge v$ occurs. The linear independence of $\Theta_{1}, \ldots, \Theta_{g(j)}$ follows. Again (11) (vi) and (vii) are obtained by Lemma 4.3. For the case $Y^{b} Y^{d} \neq \emptyset(11)$, and therefore the lemma are proved.

If $Y^{b} Y^{d}=\emptyset$ but $Y^{c} \neq \emptyset$, we prove Case I of (11) in the same way. We define $\Delta_{f(j)}$ and $\Theta_{g(j)}$ analogously as above, working with $Z_{j}^{c y}$ instead of $Z_{j}^{b y}$.

Now we carry out the induction assuming that $Y=Y^{a} \subseteq A$. By the assumption above $X_{j}^{+} \nsubseteq A$ for all $j$. Then $k>0$. Assume (11) is shown for $j-1$. It remains to show (11) for $j$ in Case I. As mentioned above, $Z_{j}$ is split into $Z_{j}^{+}=Z_{j}^{+a} Z_{j}^{+b y}$ and $Z_{j}^{-}=Z_{j}^{-a} Z_{j}^{-b y}$ such that $Y Z_{1} \ldots Z_{j}^{+} Z_{j}^{-} \ldots Z_{h}$ gives us a suitable basis of $\left\langle Y X_{1}^{+} X_{2}^{+} X_{2}^{-} \ldots X_{h}^{+} X_{h}^{-}\right\rangle$that respects

$$
\begin{aligned}
& \langle Y\rangle,\left\langle Y X_{1}^{+}\right\rangle,\left\langle Y X_{1}^{+} X_{2}^{+} X_{2}^{-}\right\rangle, \ldots,\left\langle Y X_{1}^{+} X_{2}^{+} X_{2}^{-} \ldots X_{j-1}^{+} X_{j-1}^{-}\right\rangle \\
& \left\langle Y X_{1}^{+} X_{2}^{+} X_{2}^{-} \ldots X_{j-1}^{+} X_{j-1}^{-} X_{j}^{+}\right\rangle,\left\langle Y X_{1}^{+} X_{2}^{+} X_{2}^{-} \ldots X_{j}^{+} X_{j}^{-}\right\rangle \\
& \left\langle Y X_{1}^{+} X_{2}^{+} X_{2}^{-} \ldots X_{j+1}^{+} X_{j+1}^{-}\right\rangle, \ldots,\left\langle Y X_{1}^{+} X_{2}^{+} X_{2}^{-} \ldots X_{h-1}^{+} X_{h-1}^{-}\right\rangle .
\end{aligned}
$$


The image of $X_{j}^{+}$and the image of $Z_{j}^{+}$form bases of the vector space

$$
\left\langle Y X_{1}^{+} X_{2}^{+} X_{2}^{-} \ldots X_{j-1}^{+} X_{j-1}^{-} X_{j}^{+}\right\rangle
$$

over $\left\langle Y X_{1}^{+} X_{2}^{+} X_{2}^{-} \ldots X_{j-1}^{+} X_{j-1}^{-}\right\rangle$. If $Z_{j}^{+a} Z_{j}^{+b y}=\left\{t_{1}^{j}, \ldots, t_{k}^{j}\right\}$, then by (S4) we can assume w.l.o.g. that $x_{i}^{j}=t_{i}^{j}+q_{i}^{j}$, where $q_{i}^{j} \in\left\langle Y X_{1}^{+} X_{2}^{+} X_{2}^{-} \ldots X_{j-1}^{+} X_{j-1}^{-}\right\rangle$. If $t_{i}^{j} \in Z_{j}^{+a}$, then $t_{i}^{j}$ does not occur in $W_{j-1} U_{j-1} V_{j-1}$. If $t_{i}^{j} \in Z_{j}^{+b y}$, then $t_{i}^{j} \in U_{j-1}$ and there exists some $w_{i}^{j} \in V_{j-1}$ such that $t_{i}^{j}+w_{i}^{j} \in Q^{d} Z_{2}^{e} \ldots Z_{j-1}^{e}$ but $t_{i}^{j}$ and $w_{i}^{j}$ are not in $\left\langle Y X_{1}^{+} X_{2}^{+} X_{2}^{-} \ldots X_{j-1}^{+} X_{j-1}^{-}\right\rangle$. If we rewrite $q_{i}^{j}$ with respect to $W_{j-1} U_{j-1} V_{j-1}$, then we have $x_{i}^{j}=\left(1+\gamma_{i}\right) t_{i}^{j}+\gamma_{i} w_{i}^{j}+q_{i}$ where $q_{i} \in\left\langle W_{j-1} U_{j-1} V_{j-1} \backslash\left\{t_{i}^{j}, w_{i}^{j}\right\}\right\rangle$. If $t_{i}^{j} \in Z_{j}^{+a}$, then we use the same representation with $\gamma_{i}=0$ and $w_{i}^{j}=0$. For $1 \leq i \leq k$ we define $x_{i}^{+}=t_{i}^{j}$ if $1+\gamma_{i} \neq 0$, and $x_{i}^{+}=w_{i}^{j}$ if $1+\gamma_{i}=0$. Then $x_{i}^{j}=\epsilon_{i} x_{i}^{+}+q_{i}^{+}$, where $0<\epsilon_{i}<p$ and $q_{i}^{+} \in\left\langle W_{j-1} U_{j-1} V_{j-1} \backslash\left\{x_{i}^{+}\right\}\right\rangle . x_{1}^{+}, \ldots, x_{k}^{+}$is a basis of $\left\langle Y X_{1}^{+} X_{2}^{+} X_{2}^{-} \ldots X_{j-1}^{+} X_{j-1}^{-} X_{j}^{+}\right\rangle$modulo $\left\langle Y X_{1}^{+} X_{2}^{+} X_{2}^{-} \ldots X_{j-1}^{+} X_{j-1}^{-}\right\rangle$as $x_{1}^{j}, \ldots, x_{k}^{j}$. By (S4) again w.l.o.g. $\epsilon_{i}=1$ and $q_{i}^{+} \in\left\langle W_{j-1} U_{j-1} V_{j-1} \backslash\left\{x_{1}^{+}, \ldots, x_{k}^{+}\right\}\right\rangle$. First we assume that $Z_{j}^{+b y} \neq \emptyset$. W.l.o.g. $t_{1}^{j} \in Z_{j}^{+b y}$. By (S1) we obtain $\Phi_{i}^{j}$, s that freely generate $\left\langle Y X_{1}^{+} X_{2}^{+} X_{2}^{-} \ldots X_{j}^{+} X_{j}^{-}\right\rangle$over $\left\langle Y X_{1}^{+} X_{2}^{+} X_{2}^{-} \ldots X_{j-1}^{+} X_{j-1}^{-}\right\rangle . \Phi_{i}^{j}$ is of the form

$$
\sum_{1 \leq s<t \leq k} \alpha_{s t}^{i}\left(\left(x_{s}^{j} \wedge x_{t}^{j}\right)-\left(x_{s}^{1} \wedge x_{t}^{1}\right)\right)+\sum_{1 \leq t \leq k}\left(y_{t}^{i} \wedge\left(x_{t}^{j}-x_{t}^{1}\right)\right)+\sum_{k<t \leq r}\left(y_{t}^{i} \wedge z_{t}^{j}\right)
$$

Then there are some $i$ and $s$ with $\alpha_{1 s}^{i} \neq 0$. If we rewrite $\Phi_{i}^{j}$ with respect to the suitable basis $W_{j} U_{j} V_{j}$, then $x_{1}^{+} \wedge x_{s}^{+}$occurs in the linear combination of basic commutators exactly once (with the coefficient $\alpha_{1 s} \neq 0$ ). If $x_{s}^{+} \in A$, then this is clear. Otherwise further $\left(x_{1}^{+} \wedge x_{s}^{+}\right)$'s can be produced by the $\left(x_{l}^{1} \wedge x_{q}^{1}\right)$ 's only, since $Y \subseteq A$. But $t_{1}$ or $w_{1}$ occurs in $x_{l}^{1}$ as $t_{1}+w_{1}$ only and $t_{s}$ or $w_{s}$ as $t_{s}+w_{s}$ only. Hence this is impossible. As above let $\Phi_{i}^{j}$ be $\Phi_{i}^{b}+\Phi_{i}^{c}$, and $J \in \wedge^{2} A$, be such that $\Phi_{i}^{b}+J \in N(B)$ and $\Phi_{i}^{c}-J \in N(C)$.

If $x_{s}^{+}=t_{s}^{j} \in A$, then $x_{1}^{+} \wedge x_{s}^{+}$does not occur in $\wedge^{2}\left\langle W_{j-1} U_{j-1} V_{j-1}\right\rangle+\wedge^{2} A$. If $x_{1}^{+} \in U_{j}$, then $\Delta_{f(j)}=\Phi_{i}^{b}+J$ is independent from $\Delta_{1}, \ldots, \Delta_{j-1}$ modulo $\wedge^{2} A$. If $x_{1}^{+} \in V_{j}$, then $\Theta_{g(j)}=\Phi_{i}^{c}-J$ is independent from $\Theta_{1}, \ldots, \Theta_{g(j-1)}$ modulo $\wedge^{2} A$. (11.vi) and (11.vii) follow again by Lemma 4.3 .

If $x_{s}^{+}$is not in $A$, then by Lemma 4.3 either $x_{1}^{+}$and $x_{s}^{+}$are in $U_{j}$ or they are in $V_{j}$. We construct $\Delta_{f(j)}$ or $\Theta_{g(j)}$ respectively as above. The independence of $\Delta_{f(j)}$ from $\Delta_{1}, \ldots, \Delta_{f(j-1)}$ or of $\Theta_{g(j)}$ from $\Theta_{1}, \ldots, \Theta_{g(j-1)}$ over $\wedge^{2} A$ respectively follows, since by (11.vi) and (11.vii) $x_{1}^{+} \wedge x_{s}^{+}$cannot occur in the representations of $\Delta_{1}, \ldots, \Delta_{f(j-1)}$ and of $\Theta_{1}, \ldots, \Theta_{g(j-1)}$. (11.vi) and (11.vii) follow as above.

There remains the case $Z_{j}^{+b y}=0$. That means $x_{s}^{+}=t_{s}^{+} \in A$ for all $s$ with $1 \leq s \leq k$. We use $x_{s}^{j}=x_{s}^{+}+q_{s}^{+}$, where $q_{s}^{+} \in\left\langle W_{j-1} U_{j-1} V_{j-1}\right\rangle$. Since by assumption $X_{j}^{+} \nsubseteq A$, there exists some $w \in U_{j-1} V_{j-1}$ and some $t$ such that for $1 \leq s \leq k x_{s}^{j}=x_{s}^{+}+\gamma_{s} w+q_{s}^{0}$. where $0 \leq \gamma_{s}<p, q_{s}^{0} \in\left\langle W_{j-1} U_{j-1} V_{j-1} \backslash\{w\}\right\rangle$, and $\gamma_{t} \neq 0$. By (S4) w.l.o.g. $t=1$. Note $w \notin Y$, since $Y \subseteq A$. By (S4) w.l.o.g. $\gamma_{s}=0$ if and only if $s \neq 1$. Choose $s$ such that $\alpha_{1 s} \neq 0$ in $\Phi_{i}^{j}$. Then $w \wedge x_{s}^{+}$is a new basic commutator in the representation of $\Phi_{i}^{j}$ with respect to $W_{j} U_{j} V_{j}$. Here we use $w \notin Y$. We obtain $\Delta_{f(j)}$ or $\Theta_{g(j)}$, the linear independence over $\wedge^{2} A$, and (11.vi,vii) as above. 
Lemma 6.6. Assume that $A, B$, and $C$ are in $\mathcal{S}$ and fulfill ( $\Sigma 3)$. $A$ is a common substructure of $B$ and $C$. Let $D$ be $B \star_{A} C$. Suppose that $A$ is selfsufficient in $B$ and $\operatorname{dim}(B / A)$-selfsufficient in $C$. Let $Y X_{1}^{+} X_{2}^{+} X_{2}^{-} \ldots X_{h}^{+} X_{h}^{-}$be a reduced $k$-sequence in $D$.

(i) If there exists some $j(2 \leq j \leq h)$ such that $\left\langle Y X_{1}^{+} X_{j}^{+} X_{j}^{-}\right\rangle \cap C=\left\langle Y X_{1}^{+}\right\rangle \cap C$, then $Y \subseteq B$.

(ii) If there exists some $j(2 \leq j \leq h)$ such that $\left\langle Y X_{1}^{+} X_{j}^{+} X_{j}^{-}\right\rangle \cap B=\left\langle Y X_{1}^{+}\right\rangle \cap B$, then $Y \subseteq C$.

Proof. Assume that $Y=Y^{a} Y^{b} Y^{c} Y^{d}$ is a suitable basis of $\langle Y\rangle$, and

$$
S=S^{a} S^{b y} S^{b x} S^{c x} S^{e}
$$

is a suitable extension of $Y$ for $\left\langle Y X_{1}^{+}\right\rangle$. Then (i) and (ii) have the following form:

(i) If $Z_{j}=Z_{j}^{b x} Z_{j}^{e}$ is a suitable extension of $Y S$ for $\left\langle Y X_{1}^{+} X_{j}^{+} X_{j}^{-}\right\rangle$over $\left\langle Y X_{1}^{+}\right\rangle$, then $Y \subseteq B$.

(ii) If $Z_{j}=Z_{j}^{c x} Z_{j}^{e}$ is a suitable extension of $Y S$ for $\left\langle Y X_{1}^{+} X_{j}^{+} X_{j}^{-}\right\rangle$over $\left\langle Y X_{1}^{+}\right\rangle$, then $Y \subseteq C$.

By Theorem 4.1, $D$ fulfills $(\Sigma 3)^{0}$. We show (i); (ii) follows similarly. Assume that $Y^{d}$ is choosen in such a way that for every $y_{b}$ in $S^{b y}$ there is some $y$ in $Y^{d}$ with $y=y_{b}+y_{c}$, where $y_{c}$ is in $S^{c y}$. If $Y \nsubseteq B$, then $Y^{c} Y^{d} \neq \emptyset$. Let $y$ be an element of $Y^{c} Y^{d}$. We have $y^{+}=y_{c}$ if $y=y_{b}+y_{c}$ with $y_{b} \in S^{b y}$ and $y_{c} \in S^{c y}$; otherwise $y^{+}=y$. Using (S2), we can assume that $j=2$. By (S1), $N\left(\left\langle Y X_{1}^{+} X_{2}^{+} X_{2}^{-}\right\rangle\right)$is freely generated by $\Phi_{1}^{2}, \ldots, \Phi_{r}^{2}$ over $N\left(\left\langle Y X_{1}^{+}\right\rangle\right.$, where the $\Phi_{i}^{2}$ 's are of the form

$$
\sum_{1 \leq s<t \leq k} \alpha_{s t}^{i}\left(\left(x_{s}^{2} \wedge x_{t}^{2}\right)-\left(x_{s}^{1} \wedge x_{t}^{1}\right)\right)+\sum_{1 \leq t \leq k}\left(y_{t}^{i} \wedge\left(x_{t}^{2}-x_{t}^{1}\right)\right)+\sum_{k<t \leq r}\left(y_{t}^{i} \wedge z_{t}^{2}\right) .
$$

If we rewrite the $\Phi_{i}^{2}$ 's with respect to $Y X_{1}^{+} X_{2}^{+} X_{2}^{-}$, which means the $y_{t}^{i}$ 's with respect to the basis $Y$, then we denote by $\gamma_{t}^{i}$ the coefficient of $y \wedge\left(x_{t}^{2}-x_{t}^{1}\right)$ for $1 \leq t \leq k$ and of $y \wedge z_{t}^{2}$ for $k<t \leq r$.

For $1 \leq t \leq k$ assume $x_{t}^{2}=x_{t}+x_{t}^{0}+\epsilon_{t} y^{+}$, where $x_{t} \in\left\langle Z_{2}^{b x} Z_{2}^{e}\right\rangle, x_{t}^{0}$ $\in\left\langle Y S \backslash\left\{y^{+}\right\}\right\rangle$, and for $k<t \leq r$ assume $z_{t}^{2}=z_{t}+z_{t}^{0}$, where $z_{t} \in\left\langle Z_{2}^{b y} Z_{2}^{e}\right\rangle$ and $z_{t}^{0} \in\langle Y S\rangle$. W.l.o.g. we suppose that $\left\{x_{1}, \ldots, x_{k}, z_{k+1}, \ldots, z_{r}\right\}=Z_{2}^{b y} Z_{2}^{e}$ as sets, since otherwise we could apply an appropriate well-tolerated automorphism and use (S4).

In the representation of $\Phi_{i}^{2}(1 \leq i \leq r)$ with respect to a suitable basis of $D$ that is consistent with $Y S Z_{2}^{b x} Z_{2}^{e}$ the coefficient of $y^{+} \wedge z_{t}$ is $\gamma_{t}^{i}$ and the coefficient of $y^{+} \wedge x_{t}$ is $\gamma_{t}^{i}+\sum_{s<t} \alpha_{s t}^{i} \epsilon_{s}-\sum_{t<s} \alpha_{t s}^{i} \epsilon_{s}$. There has to be some $i$ such that at least one of these coefficients is not zero. Otherwise the well-tolerated automorphism $e$ defined by $e\left(x_{t}^{j}\right)=x_{t}^{j}-\epsilon_{t} y$ and $e\left(z_{t}^{j}\right)=z_{t}^{j}$ would give a representation of all $\Phi_{i}^{j}$ as in (S1) with respect to $Y X_{1}^{+} X_{2}^{+} X_{2}^{-} \ldots X_{h}^{+} X_{h}^{-} \backslash\{y\}$. But $y^{+} \wedge x_{t}$ or $y^{+} \wedge z_{t}$ with $y^{+} \in Y^{c} Y^{d} S^{c y}$ and $x_{t}, z_{t} \in Z_{2}^{b x} Z_{2}^{e}$ cannot occur in the representation of any $\Phi_{i}^{j}$ by Lemma 4.3.

Lemma 6.7. Assume that we are in the situation of Lemma 6.4.

Let $Y X_{1}^{+} X_{2}^{+} X_{2}^{-} \ldots X_{h}^{+} X_{h}^{-}$be a reduced $k$-sequence where $0<k$ and $r,|Y|+k<$ $m$.

(i) If $\left\langle Y X_{1}^{+}\right\rangle \subseteq B$ and $\left\langle Y X_{1}^{+}\right\rangle \cap A=\langle Y\rangle \cap A$, then all $X_{j}^{+} X_{j}^{-}$are in $B$.

(ii) If $\left\langle Y X_{1}^{+}\right\rangle \subseteq C$ and $\left\langle Y X_{1}^{+}\right\rangle \cap A=\langle Y\rangle \cap A$, then all $X_{j}^{+} X_{j}^{-}$are in $C$. 
Proof. We can write the assertions in the following way:

(i) If $Y \subseteq B$ and $\left\langle Y X_{1}^{+}\right\rangle$has a suitable basis $Y Z_{1}^{+b x}$, then all $X_{j}^{+} X_{j}^{-}$are in $B$.

(ii) If $Y \subseteq C$ and $\left\langle Y X_{1}^{+}\right\rangle$has a suitable basis $Y Z_{1}^{+c x}$, then all $X_{j}^{+} X_{j}^{-}$are in $C$.

We show (i). The proof of (ii) is similar. Let $Y$ be $Y^{a} Y^{b}$, a suitable basis of $\langle Y\rangle$, and let $Y Z_{1}^{+b x} Z_{j}^{a} Z_{j}^{b x} Z_{j}^{c x} Z_{j}^{e}$ be a suitable basis of $\left\langle Y X_{1}^{+} X_{j}^{+} X_{j}^{-}\right\rangle$. W.l.o.g. $Z_{1}^{+b x}=X_{1}^{+}$. We have to show that $Z_{j}^{c x} Z_{j}^{e}=\emptyset$. Otherwise let us first assume that $Z_{j}^{c x} Z_{j}^{e} \neq \emptyset$ and $Z_{j}^{a} Z_{j}^{b x} \neq \emptyset$. Let $Z_{j}^{e}$ be $\left\{t_{1}+w_{1}, \ldots, t_{l}+w_{l}\right\}$. Note that $Y^{c} Y^{d}=\emptyset$. By Lemma $5.1 \delta\left(\left\langle\left\{w_{1}, \ldots, w_{l}\right\} Z^{c x} A\right\rangle\right)<\delta(A)$, a contradiction to the $2 m^{2}$-selfsufficiency of $A$ in $C$. Note that $r<m$.

There remains the case $Z_{j}^{a} Z_{j}^{b x}=\emptyset$. Then $Y Z_{1}^{+b x} Z_{j}^{+c x} Z_{j}^{+e}$ is a suitable basis of $\left\langle Y X_{1}^{+} X_{j}^{+}\right\rangle$. A suitable basis of $\left\langle Y X_{j}^{+}\right\rangle$has the form $Y S_{j}^{+c x} S_{j}^{+e}$. By Lemma 6.3 $S_{j}^{+e}=Z_{j}^{+e}=\emptyset$, and so $\left\langle Y X_{1}^{+} X_{j}^{+} X_{j}^{-}\right\rangle$has a suitable basis $Y Z_{1}^{+b x} Z_{j}^{+c x} Z_{j}^{-c x} Z_{j}^{-e}$, where $X_{1}^{+}=Z_{1}^{+b x}$ and $X_{j}^{+}=Z_{j}^{+c x}$. By Lemma $6.6 Y \subseteq C$, and therefore $Y \subseteq A$.

Next we show $Z_{j}^{-c x}=\emptyset$. We use $\left\{t_{1}+w_{1}, \ldots, t_{l}+w_{l}\right\}$ for $Z_{j}^{-e}$, where $t_{i} \in B$ and $w_{i} \in C$. Remember that $X_{1}^{+}=Z_{1}^{+b x}$ and $X_{j}^{+}=Z_{j}^{+c x}$. By (S3) and (S1) we have that $\left\langle Y X_{j}^{+} X_{1}^{+} Z_{j}^{-c x} Z_{j}^{-e}\right\rangle$ is a minimal extension of $\left\langle Y X_{j}^{+}\right\rangle .\left|Z^{-c y}\right|<r$, since $0<k$. If $Z^{-c x} \neq \emptyset$, then by Lemma $5.1(1) \operatorname{dim}\left(N\left(\left\langle\left\{t_{1}, \ldots, t_{l}\right\} X_{1}^{+} A\right\rangle\right)\right)-\operatorname{dim}(N(A))>$ $\left|X_{1}^{+}\right|+l=k+l$. It follows that $\delta\left(\left\langle\left\{t_{1}, \ldots, t_{l}\right\} X_{1}^{+} A\right\rangle\right)<\delta(A)$, a contradiction to the selfsufficiency of $A$ in $B$.

We have shown that

$$
Y X_{1}^{+} X_{j}^{+} X_{j}^{-}
$$

is a suitable basis. We write $Y^{a} X_{1}^{+b x} X_{j}^{+c x} X_{j}^{-e}$ to indicate the nature of the single parts. As above, by (S1) and (S3) $\left\langle Y^{a} X_{j}^{+c x} X_{1}^{+b x} X_{j}^{-e}\right\rangle$ is a minimal extension of $\left\langle Y^{a} X_{j}^{+c x}\right\rangle$. By Lemma 5.1 (6) applied to $\left\langle Y^{a} X_{j}^{+c x} X_{1}^{+b x} X_{j}^{-e}\right\rangle$ over $\left\langle Y^{a} X_{j}^{+c x}\right\rangle$ we obtain

$$
\operatorname{dim}\left(N\left(\left\langle X_{1}^{+b x}\left\{t_{1}, \ldots, t_{r-k}\right\} A\right\rangle\right)\right)-\operatorname{dim}(N(A)) \geq r .
$$

Hence $\delta\left(\left\langle X_{1}^{+b x}\left\{t_{1}, \ldots, t_{r-k}\right\} A\right\rangle\right) \leq \delta(A)$. Since $B$ is a minimal extension of $A$, equality follows, and $\left\langle X_{1}^{+b x}\left\{t_{1}, \ldots, t_{r-k}\right\} A\right\rangle=B$.

Let us consider the free generators $\Phi_{1}^{j}, \ldots, \Phi_{r}^{j}$ of $N\left(\left\langle Y^{a} X_{1}^{+b x} X_{j}^{+c x} X_{j}^{-e}\right\rangle\right)$ over $N\left(\left\langle Y^{a} X_{1}^{+b x}\right\rangle\right)$ given by (S1). By Lemma 4.3 there are $J_{i} \in \wedge^{2} A$ such that $\Phi_{i}^{j}=$ $\Phi_{i}^{c}-\Phi_{i}^{b}, \Phi_{i}^{b}+J_{i} \in N(B)$, and $\Phi_{i}^{c}+J_{i} \in N(C)$, where

$$
\begin{gathered}
\Phi_{i}^{b}=\sum_{1 \leq t<s \leq k} \alpha_{t s}^{i}\left(x_{t}^{1} \wedge x_{s}^{1}\right)+\sum_{1 \leq t \leq k}\left(y_{t}^{i} \wedge x_{t}^{1}\right)+\sum_{k<t \leq r}\left(y_{t}^{i} \wedge-t_{t-k}\right), \\
\Phi_{i}^{c}=\sum_{1 \leq t<s \leq k} \alpha_{t s}^{i}\left(x_{t}^{j} \wedge x_{s}^{j}\right)+\sum_{1 \leq t \leq k}\left(y_{t}^{i} \wedge x_{t}^{j}\right)+\sum_{k<t \leq r}\left(y_{t}^{i} \wedge w_{t-k}\right) .
\end{gathered}
$$

Note that

$$
z_{k+l}^{j}=t_{l}+w_{l} .
$$

Since $\Phi_{1}^{j}, \ldots, \Phi_{r}^{j}$ are linearly independent over $N\left(\left\langle Y^{a} X_{1}^{+b x}\right\rangle\right)$, every non-trivial linear combination of them contains some basic commutator $x_{t}^{j} \wedge x_{s}^{j}$ or $a \wedge x_{t}^{j}$ or $a \wedge t_{s}$ for some $a \in Y$. Hence this linear combination also contains $x_{t}^{1} \wedge x_{s}^{1}$ or $a \wedge x_{t}^{1}$ or $a \wedge w_{s}$ respectively. Therefore $\Phi_{1}^{c}+J_{1}, \ldots, \Phi_{r}^{c}+J_{r}$ are linearly independent over $\wedge^{2} A$ and $\Phi_{1}^{b}+J_{1}, \ldots, \Phi_{r}^{b}+J_{r}$ are linearly independent over $\wedge^{2} A$. Since $B=$ 
$\left\langle X_{1}^{+b x}\left\{t_{1}, \ldots, t_{r-k}\right\} A\right\rangle$ and $\delta(B)=\delta(A), \Phi_{1}^{b}+J_{1}, \ldots, \Phi_{r}^{b}+J_{r}$ freely generate $N(B)$ over $N(A)$. Since $A$ is $2 m^{2}$-selfsufficient in $C$, we obtain that $\Phi_{1}^{c}, \ldots, \Phi_{r}^{c}$ freely generate $N\left(\left\langle X_{j}^{c x}\left\{w_{1}, \ldots, w_{r-k}\right\} A\right\rangle\right.$ over $N(A)$. Hence $f\left(x_{i}^{1}\right)=x_{i}^{j}$ for $1 \leq i \leq k$, $f\left(t_{i}\right)=-w_{i}$ for $1 \leq i \leq r-k$, and $f(a)=a$ for $a \in A$ induces an isomorphism of $B$ into $C$ that is the identity on $A$ and can be extended to the corresponding structures in $\mathcal{S}$. This contradicts the assumption of the lemma, that $B$ is not realized in $C$.

Proof of Lemma 6.4. Let $Y X_{1}^{+} X_{2}^{+} X_{2}^{-} \ldots X_{h}^{+} X_{h}^{-}$be a reduced $k$-sequence in $D$ with $r,|Y|+k<m$. Assume $Y$ is a suitable basis of $\langle Y\rangle$.

Case 1. $k>0$ and there exists some $j$ such that $\left\langle Y X_{j}^{+}\right\rangle \cap C=\langle Y\rangle \cap C$. By (S3) we can assume w.l.o.g. that $j=1$.

Case 1.1. There exists an $i$ such that $2 \leq i \leq h,\left\langle Y X_{1}^{+} X_{i}^{+} X_{i}^{-}\right\rangle \cap C=\left\langle Y X_{1}^{+}\right\rangle \cap C$.

By Lemma 6.6 $Y \subseteq B$. By (S2) we can assume w.l.o.g. that $i=2$. By the assumption we have $\left\langle Y X_{1}^{+}\right\rangle \cap C=\langle Y\rangle \cap C$ and $\left\langle Y X_{1}^{+} X_{2}^{+}\right\rangle \cap C=\left\langle Y X_{1}^{+}\right\rangle \cap C$. Lemma 6.3 implies $\left\langle Y X_{1}^{+}\right\rangle=\langle Y\rangle+\left(\left\langle Y X_{1}^{+}\right\rangle \cap B\right)$. Using $Y \subseteq B$ we get $\left\langle Y X_{1}^{+}\right\rangle \subseteq B$. Then $\left\langle Y X_{1}^{+}\right\rangle \cap C=\langle Y\rangle \cap C$ implies $\left\langle Y X_{1}^{+}\right\rangle \cap A=\langle Y\rangle \cap A$. Hence by Lemma 6.7 all $X_{j}^{+} X_{j}^{-} \subseteq B$. The assertion follows from $(\Sigma 4)^{m}$ for $B$.

Case 1.2. There exists an $i$ such that $2 \leq i \leq h$, and $\left\langle Y X_{1}^{+} X_{i}^{+} X_{i}^{-}\right\rangle \cap B=\left\langle Y X_{1}^{+}\right\rangle \cap$ $B$.

By Lemma 6.6 $Y \subseteq C$. By (S2) we can assume w.l.o.g. that $i=2$. By the assumptions we have $\left\langle Y X_{1}^{+}\right\rangle \cap C=\langle Y\rangle \cap C$, and $\left\langle Y X_{1}^{+} X_{2}^{+}\right\rangle \cap B=\left\langle Y X_{1}^{+}\right\rangle \cap B$. Note that this implies $\left\langle Y X_{2}^{+}\right\rangle \cap B=\langle Y\rangle \cap B$. Lemma 6.3 implies $\left\langle Y X_{2}^{+}\right\rangle=$ $\langle Y\rangle+\left(\left\langle Y X_{2}^{+}\right\rangle \cap C\right)$. Using $Y \subseteq C$ we get $\left\langle Y X_{2}^{+}\right\rangle \subseteq C$. Since $\left\langle Y X_{2}^{+}\right\rangle \cap B=\langle Y\rangle \cap B$ we get $\left\langle Y X_{2}^{+}\right\rangle \cap A=\langle Y\rangle \cap A$. By (S3) we can exchange $X_{1}^{+}$and $X_{2}^{+}$. Hence by Lemma 6.7 we get $X_{1}^{+} \subseteq C$, a contradiction to the general assumption of Case 1 .

Case 1.3. For all $i$ with $2 \leq i \leq h$ we have $\left\langle Y X_{1}^{+} X_{i}^{+} X_{i}^{-}\right\rangle \cap C \neq\left\langle Y X_{1}^{+}\right\rangle \cap C$ and $\left\langle Y X_{1}^{+} X_{i}^{+} X_{i}^{-}\right\rangle \cap B \neq\left\langle Y X_{1}^{+}\right\rangle \cap B$.

In this case the assertion follows from Lemma 6.5.

Case 2. The roles of $B$ and $C$ are exchanged in Case 1.

The proof is completely symmetric.

Case 3. $k>0$ and for every $j$ with $1 \leq j \leq h$ we have neither $\left\langle Y X_{j}^{+}\right\rangle \cap C=\langle Y\rangle \cap C$ nor $\left\langle Y X_{j}^{+}\right\rangle \cap B=\langle Y\rangle \cap B$.

We get $\left\langle Y X_{1}^{+} X_{j}^{+} X_{j}^{-}\right\rangle \cap C \neq\left\langle Y X_{1}^{+}\right\rangle \cap C$ and $\left\langle Y X_{1}^{+} X_{j}^{+} X_{j}^{-}\right\rangle \cap B \neq\left\langle Y X_{1}^{+}\right\rangle \cap B$. The assertion follows from Lemma 6.5.

Case $4 . k=0$ and for every $j$ with $2 \leq j \leq h$ we have neither $\left\langle Y X_{j}^{-}\right\rangle \cap C=\langle Y\rangle \cap C$ nor $\left\langle Y X_{j}^{-}\right\rangle \cap B=\langle Y\rangle \cap B$.

In this case the assertion follows immediately from Lemma 6.5.

Case 5. $k=0$ and there is some $j$ with $2 \leq j \leq h$ such that $\left\langle Y X_{j}^{-}\right\rangle \cap C=\langle Y\rangle \cap C$. 
In this case $\left\langle Y X_{j}^{-}\right\rangle$has a suitable basis $Y S_{j}^{b x} S_{j}^{e}$. By Lemma $6.6 Y \subseteq B$. By (S2) w.l.o.g. $j=2$. Let $Y S_{i}^{a} S_{i}^{b x} S_{i}^{c x} S_{i}^{e}$ be a suitable basis of $\left\langle Y X_{i}^{-}\right\rangle$for $2 \leq i \leq h$. Since $Y^{d}=\emptyset$ there is no $S_{i}^{b y}$. Let $S_{i}^{e}$ be $\left\{t_{1}+w_{1}, \ldots, t_{l}+w_{l}\right\}$, where $t_{1}, \ldots, t_{l}$ are in $B$, $w_{1}, \ldots, w_{l}$ are in $C$, and $t_{1}, \ldots, t_{l}, w_{1}, \ldots, w_{l}$ are linearly independent over $A$. First assume $Y^{b} \neq \emptyset$. If $S_{i}^{a} S_{i}^{b x}=\emptyset$, then $Y \subseteq C$ by Lemma 6.6 , a contradiction to $Y^{b} \neq \emptyset$. Hence $S_{i}^{a} S_{i}^{b x} \neq \emptyset$. If $S_{i}^{c x} S_{i}^{e} \neq \emptyset$, then $\operatorname{dim}\left(N\left(\left\langle S_{i}^{c x}\left\{w_{1}, \ldots, w_{l}\right\} A\right\rangle\right)\right)-\operatorname{dim}(N(A))>$ $\left|S_{i}^{c x}\right|+l$ by Lemma 5.1. Therefore $\delta\left(\left\langle S_{i}^{c x}\left\{w_{1}, \ldots, w_{l}\right\} A\right\rangle\right)<\delta(A)$, a contradiction to the $2 m^{2}$-selfsufficiency of $A$ in $C$. Note that $r<m$. We have shown that $S_{i}^{c x} S_{i}^{e}=\emptyset$ for every $i$. Hence all $X_{i}^{-}$are in $B$. The assertion follows from $(\Sigma 4)^{m}$ for $B$.

There remains the case $Y=Y^{a} \subseteq A$. A suitable basis $Y S_{i}^{e}$ for some $\left\langle Y X_{i}^{-}\right\rangle$is impossible. In this case we could assume $X_{i}^{-}=S_{i}^{e}$ by (S4) and apply Lemma 4.4 to the situation described in (S1). We would get a contradiction to $\delta(\langle Y\rangle)=\delta\left(\left\langle Y X_{i}^{-}\right\rangle\right)$ Then by Lemma 5.2 every $X_{i}^{-}$is in $B$. The assertion follows from $(\Sigma 4)^{m}$ for $B$.

Case 6. $k=0$ and there is some $j$ with $2 \leq j \leq h$ such that $\left\langle Y X_{j}^{-}\right\rangle \cap B=\langle Y\rangle \cap B$.

A similar proof as for Case 5 works.

Lemma 6.8. Assume $A, B$, and $C$ are finite structures in $\mathcal{S}$ that satisfy ( $\Sigma 3)$ and $(\Sigma 4)$. Furthermore $A$ is a common substructure of $B$ and $C, B$ is a minimal extension of $A$ that is not realized in $C$, and $A$ is n-selfsufficient in $C$, where $n \geq 2(2 \operatorname{dim}(B)+1)^{2}$. Let $D$ be $B \star_{A} C$.

Then $C$ is selfsufficient in $D, B$ is n-selfsufficient in $D$, and $D$ satisfies $(\Sigma 3)$ and $(\Sigma 4)$.

Proof. By Theorem 4.1 we have everything except $(\Sigma 4)$ for $D$. Note that

$$
2(2 \operatorname{dim}(B)+1)^{2} \geq \operatorname{dim}(B / A)+2 .
$$

By Lemma 6.4 we obtain $(\Sigma 4)^{2 \operatorname{dim}(B)+1}$. That means for every reduced $k$-sequence with $|Y|+k, r \leq 2 \operatorname{dim}(B)$ we have $h \leq 2 \max \{r,|Y|+k\}+1$.

Now we consider reduced $k$-sequences $Y X_{1}^{+} X_{2}^{+} X_{2}^{-} \ldots X_{h}^{+} X_{h}^{-}$with $r>2 \operatorname{dim}(B)$ or $|Y|+k>2 \operatorname{dim}(B)$. Assume that $Y$ is a suitable basis of $\langle Y\rangle$. We choose

$$
Z_{j}=Z_{j}^{a} Z_{j}^{b y} Z_{j}^{b x} Z_{j}^{c x} Z_{j}^{e}
$$

such that $Y Z_{1} \ldots Z_{h}$ gives us a suitable basis for $\left\langle Y X_{1}^{+} X_{2}^{+} X_{2}^{-} \ldots X_{h}^{+} X_{h}^{-}\right\rangle$that respects $\langle Y\rangle,\left\langle Y X_{1}^{+}\right\rangle, \ldots,\left\langle Y X_{1}^{+} X_{2}^{+} X_{2}^{-} \ldots X_{h-1}^{+} X_{h-1}^{-}\right\rangle$.

If $h \leq 2 \operatorname{dim}(B)+1$, then $(\Sigma 4)$ is fulfilled by this sequence. In the case $h>$ $2 \operatorname{dim}(B)+1$ we will show that the whole reduced $k$-sequence is contained in $C$ and the assertion follows from $(\Sigma 4)$ for $C$.

If $h>2 \operatorname{dim}(B)+1$, then there are different $j_{0}, j_{1}$ with $1 \leq j_{0}<j_{1} \leq 2 \operatorname{dim}(B)+2$, such that

$$
\left\langle Y X_{1}^{+} X_{2}^{+} X_{2}^{-} \ldots X_{j_{0}}^{+} X_{j_{0}}^{-}\right\rangle \cap B=\left\langle Y X_{1}^{+} X_{2}^{+} X_{2}^{-} \ldots X_{j_{0}-1}^{+} X_{j_{0}-1}^{-}\right\rangle \cap B
$$

and

$$
\left\langle Y X_{1}^{+} X_{2}^{+} X_{2}^{-} \ldots X_{j_{1}}^{+} X_{j_{1}}^{-}\right\rangle \cap B=\left\langle Y X_{1}^{+} X_{2}^{+} X_{2}^{-} \ldots X_{j_{1}-1}^{+} X_{j_{1}-1}^{-}\right\rangle \cap B .
$$

After an appropriate application of (S2) and (S3) we can assume that $j_{0}=1$ and $j_{1}=2$. Then $\left\langle Y X_{1}^{+}\right\rangle \cap B=\langle Y\rangle \cap B$ and $\left\langle Y X_{1}^{+} X_{2}^{+} X_{2}^{-}\right\rangle \cap B=\left\langle Y X_{1}^{+}\right\rangle \cap B$. Hence by Lemma $6.6 Y \subseteq C$. Furthermore, $\left\langle Y X_{1}^{+} X_{2}^{+}\right\rangle \cap B=\langle Y\rangle \cap B$. By Lemma 6.3 $\left\langle Y X_{1}^{+} X_{2}^{+}\right\rangle=\langle Y\rangle+\left(\left\langle Y X_{1}^{+} X_{2}^{+}\right\rangle \cap C\right)$. The last two equations imply that $\left\langle Y X_{1}^{+} X_{2}^{+}\right\rangle$ 
has a suitable basis $Y Z_{1}^{+c x} Z_{2}^{+c x} \subseteq C$, where $Y Z_{1}^{+c x}$ is a suitable basis of $\left\langle Y X_{1}^{+}\right\rangle$. Now we know that $Y \subseteq C$ and $Z_{1}=Z_{1}^{+c x}$. Choose $j$ minimal such that $Z_{j} \nsubseteq \subseteq C$. Then $Y S_{1}^{+c x} Z_{2} \ldots Z_{j}$ is a suitable basis of $\left\langle Y X_{1}^{+} X_{2}^{+} X_{2}^{-} \ldots X_{j}^{+} X_{j}^{-}\right\rangle$. Let $Z_{j}$ be $Z_{j}^{a} Z_{j}^{b x} Z_{j}^{c x} Z_{j}^{e}$. Note that $Z_{j}^{b y}=\emptyset$ and $Z_{j}^{b x} Z_{j}^{e} \neq \emptyset$, by the choice of $j$.

First assume $Z_{j}^{a} Z_{j}^{c x} \neq \emptyset$. Note that $\operatorname{dim}(B)<r$ implies $Z_{j}^{c x} \neq \emptyset$. By Lemma 5.1 (1) we obtain $\operatorname{dim}\left(N\left(\left\langle Z_{j}^{b x}\left\{t_{1}, \ldots t_{l}\right\} A\right\rangle\right)\right)-\operatorname{dim}(N(A))>\left|Z_{j}^{b x}\right|+l$, where $Z_{j}^{e}=$ $\left\{t_{1}+w_{1}, \ldots, t_{l}+w_{l}\right\}$ and $Z_{j}^{b x}\left\{t_{1}, \ldots, t_{l}\right\} \subseteq B$ is linearly independent over $A$. Then $\delta\left(\left\langle Z_{j}^{b x}\left\{t_{1}, \ldots, t_{l}\right\} A\right\rangle\right)<\delta(A)$, a contradiction to the selfsufficiency of $A$ in $B$.

If $Z_{j}^{a} Z_{j}^{c x}=\emptyset$, then $r \leq \operatorname{dim}(B)$. Hence by the assumption $|Y|+k>2 \operatorname{dim}(B)$. Since $k \leq r \leq \operatorname{dim}(B)$, we get $|Y|>\operatorname{dim}(B)$ and therefore $Y^{c} \neq \emptyset$. On the other hand, by Lemma 6.6 we have $Y \subseteq B$, a contradiction to $Y^{c} \neq \emptyset$. We have shown that all $X_{j}^{+} X_{j}^{-}$are in $C$. The assertion follows from $(\Sigma 4)$ for $C$.

The final theorem summarizes the efforts of this section and formulates the result for further applications.

Theorem 6.9 (Second Amalgamation Theorem). Assume that $A, B$, and $C$ are finite structures in $\mathcal{S}$ that satisfy $(\Sigma 3)$ and $(\Sigma 4)$. Suppose that $A$ is a common substructure of $B$ and $C, A$ is selfsufficient in $B$, and $A$ is $(\operatorname{dim}(B / A)+n)$-selfsufficient in $C$, where $n \geq 2(2 \operatorname{dim}(B)+1)^{2}$.

Then there exists an amalgam $D$ of $B$ and $C$ over $A$ such that $D$ fulfills $(\Sigma 3)$ and ( $\Sigma 4), C$ is selfsufficient in $D$, and $B$ is n-selfsufficient in $D$. If $A$ is selfsufficient in $C$, then $B$ is selfsufficient in $D$.

Proof. We decompose B into a nonrefinable tower $A=B_{0} \subset B_{1} \subset \ldots \subset B_{n-1} \subset$ $B_{n}=B$ of subspaces such that each $B_{i}$ is selfsufficient in $B$.

First we assume that we have shown the assertion for all cases where $n=1$. We prove the theorem by induction on $n$. By induction hypothesis there exists an amalgam $D_{n-1}$ of $B_{n-1}$ and $C$ over $A$ such that $D_{n-1}$ satisfies $(\Sigma 3)$ and $(\Sigma 4), C$ is selfsufficient in $D_{i-1}$, and $B_{n-1}$ is $\left(\operatorname{dim}\left(B_{n} / B_{n-1}\right)+n\right)$-selfsufficient in $D_{n-1}$. Now we apply the assertion for the case $n=1$ to the following situation: $B_{n-1}$ plays the role of $A, B$ plays the role of $B$, and $D_{n-1}$ plays the role of $C$. The $D$ we obtain has the desired properties. $C$ is selfsufficient in $D$ by Lemma 3.10.

Now we consider the case $n=1$. Then $\delta(A) \leq \delta(B) \leq \delta(A)+1$. If $\delta(A)=\delta(B)$, then $B$ is a minimal extension of $A$. If there is a realization $B^{+}$of $B$ in $C$ over $A$, then take $D=C$. The assertion follows. Note that $B^{+}$is $n$-selfsufficient in $C$, since $\delta(B)=\delta(A)$ and $A$ is $(\operatorname{dim}(B / A)+n)$-selfsufficient in $C$. If there is no realization of $B$ in $C$, then we apply Lemma 6.8 .

There remains the case $\delta(B)=\delta(A)+1$. Then $\operatorname{dim}(B)=\operatorname{dim}(A)+1$. Again we take $D$ as $B \star_{A} C$. By Theorem 4.1 we have $(\Sigma 3)$ for $D$, selfsufficiency of $C$ in $D$, and $n$-selfsufficiency of $B$ in $D$. We show $(\Sigma 4)$. Note that for every $b \in B \backslash A$ we obtain $\langle A\{b\}\rangle=B$. Furthermore, $D=\langle C\{b\}\rangle$ for every $b \in D \backslash C$. Let $Y X_{1}^{+} X_{2}^{+} X_{2}^{-} \ldots X_{h}^{+} X_{h}^{-}$be a reduced $k$-sequence in $D$. Our aim is to prove that the sequence lies completely in $C$. Then $(\Sigma 4)$ for $C$ implies $(\Sigma 4)$ for $D$. If $Y \nsubseteq C$, then in $(\mathrm{S} 1)$ there is some

$$
\Phi_{i}^{2} \in N\left(\left\langle Y X_{1}^{+} X_{2}^{+} X_{2}^{-}\right\rangle\right) \backslash N\left(\left\langle Y X_{1}^{+}\right\rangle\right)
$$

such that $y_{t}^{i} \notin C$ for some $t$. By (S4) we can consider a suitable basis $\left\{y_{t}^{i}\right\} Y^{0} Z_{1} Z_{2}$ in $D$, where $\left\{y_{t}^{i}\right\} Y^{0}=Y$ and $Y^{0}, Z_{1}, Z_{2} \subseteq C, X_{1}^{+} \subseteq\left\langle\left\{y_{t}^{i}\right\} Z_{1}\right\rangle$, and $X_{2}^{+} X_{2}^{-}=Z_{2}$. If we represent $\Phi_{i}^{2}$ with respect to this basis, we obtain a non-vanishing summand 
$\left(y_{t}^{i} \wedge x_{t}^{2}\right)$ or $\left(y_{t}^{i} \wedge z_{t}^{2}\right)$. This is the only occurrence of this basic commutator. We obtain a contradiction, since in $D$ no element of $D \backslash C$ is involved in a relation. The same argument works for $Y \subseteq C$ and $x_{i}^{j} \notin C$ or $z_{i}^{j} \notin C$ for some $i$ and $j$. If $x_{i}^{j} \notin C$, then by (S3) and (S4) w.l.o.g. $j=i=1$. Let $Y\left\{x_{1}^{1}\right\} Z_{1} Z_{2}$ be a basis of $\left\langle Y X_{1}^{+} X_{2}^{+} X_{2}^{-}\right\rangle$, where $Z_{1}, Z_{2} \subseteq C$, and $Y\left\{x_{1}^{1}\right\} Z_{1}$ is a basis of $\left\langle Y X_{1}^{1}\right\rangle$. By (S4) w.l.o.g. $\left\{x_{1}^{1}\right\} Z_{1}=X_{1}^{+}$. In (S1) there exists some $\Phi_{i}^{2}$ with $\alpha_{1 s}^{i} \neq 0$ for some $s$. If we rewrite $\Phi_{i}^{2}$ with respect to the basis above, then it contains $x_{1}^{1} \wedge x_{s}^{1}$ exactly once with the coefficient $\alpha_{1 s}^{i}$. Again we have a contradiction. If $Y \subseteq C$ and all $X_{j}^{+} \subseteq C$, but $z_{i}^{j} \notin C$, then by (S2) and (S4) w.l.o.g. $j=2, i=k+1$, and $z_{k+l}^{2} \in C$ for $2 \leq l \leq r-k$. Choose $\Phi_{i}^{2}$ with $y_{k+1}^{i} \neq 0$. Then the representation of $\Phi_{i}^{2}$ with respect to $Y X_{1}^{+} X_{2}^{+} X_{2}^{-}$contains $y_{k+1}^{i} \wedge z_{k+1}^{2}$ exactly once. Since we can assume that $y_{k+1}^{i} \in Y$, we obtain the desired contradiction.

\section{Construction of the Group}

We now come back to our category of groups, that is to the category $\mathcal{G}$. Let $H$ be a subgroup of $G$, with $G, H \in \mathcal{G}$. Then we say that $H$ is selfsufficient in $G$ if this is true for the corresponding structures in $\mathcal{S}$.

Now let $\Sigma$ be the following elementary theory formulated in the pure group language. Let $G$ be any model of $\Sigma$ and let $M$ be the corresponding structure in $\mathcal{S}$. (We still have to show its existence.) The axioms of $\Sigma$ express the following:

( $\Sigma 1): G$ is a nilpotent group of class 2 and of exponent $p>2 . p$ is a fixed prime.

( 2$): \forall x \notin Z(G) \forall z \in Z(G) \exists y([x, y]=z)$.

$(\Sigma 3)$ : For every finite subspace $H$ of the corresponding $\mathcal{S}$-structure $M$ we have $\delta(H)=\operatorname{dim}(H)$ if $\operatorname{dim}(H) \leq 3$ and $\delta(H) \geq 3$ otherwise.

$(\Sigma 4)$ : For every reduced $k$-sequence $Y X_{1}^{+} X_{2}^{+} X_{2}^{-} \ldots X_{h}^{+} X_{h}^{-}$in the corresponding structure $M$ of $\mathcal{S}$ we have $h \leq 2 \max \left(k+|Y|,\left|X_{2}^{+}\right|+\left|X_{2}^{-}\right|\right)+1$.

$(\Sigma 5)$ : Let $A$ and $B$ be finite $\mathcal{S}$-structures that satisfy $(\Sigma 3)$ and $(\Sigma 4)$. Suppose that $A$ is selfsufficient in $B$ and $(\operatorname{dim}(\bar{B} / \bar{A})+n)$-selfsufficient in $M$, where $n \geq 2(2 \operatorname{dim}(\bar{B})+1)^{2}$. Then there exists an embedding of $B$ in $M$ that extends the embedding of $A$ in $M$, such that the image of $B$ is $n$-selfsufficient in $G$.

Note that axioms $(\Sigma 3)-(\Sigma 5)$ are to be expressed in the language of groups, but refer to the properties of the corresponding structure $M$ in the category $\mathcal{S}$. Using the Second Amalgamation Theorem, we will prove the consistency, the completeness, and the uncountable categoricity of $\Sigma$.

Lemma 7.1. Assume that $M$ is in $\mathcal{S}$ with the properties $(\Sigma 3)$ and $(\Sigma 4)$. Let $g$ be in $M$ and $b \in \wedge^{2} M$ be such that there is no $h$ in $M$ with $(g \wedge h)-b \in N(M)$. Define $M^{+}$in the following way: $M^{+}=\langle a\rangle \oplus M$ and $N\left(M^{+}\right)=\langle(g \wedge a)-b\rangle \oplus N(M)$

Then $M$ is selfsufficient in $M^{+}$, and $M^{+}$satisfies $(\Sigma 3)$ and $(\Sigma 4)$.

Proof. We obtain $\delta(M)=\delta\left(M^{+}\right)$by construction. Therefore $M$ is selfsufficient in $M^{+}$. From the assumption that $(g \wedge x)-b \in N(M)$ has no solution it follows that $\delta(M) \geq 3$.

Next we show ( $\Sigma 3)$. Let $K \subseteq M^{+}$. If $K \subseteq M$, then we are done. Therefore suppose that $K \nsubseteq M$. Let $K_{1}$ be $K \cap M$. Note that $M^{+}$can be defined using any $a+h$, where $h \in M$, and the relation $(g \wedge(a+h))-(b+(g \wedge h))$ instead of $(g \wedge a)-b$. Also $(g \wedge x)-(b+(g \wedge h)) \in N(M)$ has no solution in $M$. Now $\delta(K)=\delta\left(K_{1}\right)+1-\operatorname{dim}\left(N(K) / N\left(K_{1}\right)\right)$. By definition $\operatorname{dim}\left(N(K) / N\left(K_{1}\right)\right) \leq 1$. If 
$\operatorname{dim}\left(K_{1}\right) \geq 3$, then the assertion follows. If $\operatorname{dim}\left(K_{1}\right) \leq 2$, then $\operatorname{dim}\left(N\left(K_{1}\right)\right)=0$ and the only possibility to obtain a relation in $N(K)$ is the following: As noted above, w.l.o.g. $K=\left\langle a, g_{1}, g_{2}\right\rangle$, where $g_{1}, g_{2} \in M$ and are linearly independent. Then w.l.o.g. $g_{1}=g$. A relation would have the form $(g \wedge a)-\left(g \wedge r g_{2}\right) \in N(K)$. This would imply a solution of $(g \wedge x)-b \in N(M)$, a contradiction. $(\Sigma 3)$ is shown.

To show $(\Sigma 4)$ let $Y X_{1}^{+} X_{2}^{+} X_{2}^{-} \ldots X_{h}^{+} X_{h}^{-}$be a reduced $k$-sequence that is not completely in $M$. Note that $r=1$ is impossible, since by $(\Sigma 3)$ relations of the form $y \wedge z$ do not exist.

First we assume that $Y \nsubseteq M$. Then w.l.o.g. $a \in Y$, and $Y \backslash\{a\} \subseteq M$. We fix some $j$ with $2 \leq j \leq h$ and consider the $\Phi_{i}^{j}$ 's in (S1):

$$
\sum_{1 \leq s<t \leq k} \alpha_{s t}^{i}\left(\left(x_{s}^{j} \wedge x_{t}^{j}\right)-\left(x_{s}^{1} \wedge x_{t}^{1}\right)\right)+\sum_{1 \leq t \leq k}\left(y_{t}^{i} \wedge\left(x_{t}^{j}-x_{t}^{1}\right)\right)+\sum_{k<t \leq r}\left(y_{t}^{i} \wedge z_{t}^{j}\right) .
$$

After the application of an appropriate well-tolerated automorphism we can assume that $X_{j}^{+} \subseteq M$. Since all of $Y$ must be used in (S1), there is some $i_{j}$ such that $y_{t}^{i_{j}} \neq 0$ for some $t$ with the property that $y_{t}^{i_{j}}$ involves $a$ in its representation as a linear combination over $Y$. Now we rewrite $\Phi_{i_{j}}^{j}$ with respect to a basis $Y Z_{1}^{+} X_{j}^{+} Z_{j}^{-}$, where $Z_{1}^{+} X_{j}^{+} Z_{j}^{-} \subseteq M, Z_{1}^{+}$is a basis of $\left\langle X_{1}^{+}\right\rangle$modulo $\langle\{a\}\rangle$, and $Z_{j}^{-}$is a basis of $\left\langle X_{j}^{-}\right\rangle$ modulo $\langle\{a\}\rangle$. Then $\Phi_{i_{j}}^{j}=\left(a \wedge u_{j}\right)+\left(a \wedge v_{j}\right)+w_{j}$, where $u_{j} \neq 0, u_{j} \in\left\langle X_{j}^{+} Z_{j}^{-}\right\rangle$, $v_{j} \in\left\langle Y \backslash\{a\} Z_{1}^{+}\right\rangle$, and $w_{j} \in \wedge^{2}\left\langle(Y \backslash\{a\}) Z_{1}^{+} X_{j}^{+} Z_{j}^{-}\right\rangle$. Then $u_{j} \neq 0, u_{j}+v_{j} \neq 0$, and $\Phi_{i_{j}}^{j}$ has to be a free generator of $N\left(M^{+}\right)$over $N(M)$. That means $\Phi_{i_{j}}^{j}$ is of the form $r(a \wedge g)-b_{1}$, where $0<r<p$ and $b_{1} \in \wedge^{2} M$. It follows that $h \leq 2$, since it is not possible that $u_{2}+v_{2}, u_{3}+v_{3} \in\langle g\rangle$.

Now we can assume that $Y \subseteq M$. Next we consider the case $X_{j}^{+} \subseteq M$ for some $j$. By (S3) w.l.o.g. $X_{1}^{+} \subseteq M$. The case $k=0$ is included. By assumption and by (S2) w.l.o.g. $X_{2} \nsubseteq \subseteq M$. We replace $X_{2}$ by a basis $a W$ such that $\left\langle X_{2}\right\rangle=\langle a W\rangle$ and $W \subseteq$ $M$. Since $\left\langle Y X_{1}^{+} X_{2}\right\rangle$ is a minimal extension of $\left\langle Y X_{1}^{+}\right\rangle$and $\left.\left|X_{2}\right|=r\right\rangle 1$, there are $\Phi_{i_{1}}^{2}, \Phi_{i_{2}}^{2} \in N\left(\left\langle Y X_{1}^{+} X_{2}\right\rangle\right)$ linearly independent over $N\left(\left\langle Y X_{1}^{+} W\right\rangle\right)$. This contradicts the assumption that $N\left(M^{+}\right)$is generated by a single relation over $N(M)$.

Finally, we assume $Y \subseteq M$ but all $X_{j}^{+} \nsubseteq M$. We consider $j>1$. Using (S4), we can suppose w.l.o.g. that $x_{1}^{j}=a$ and $x_{2}^{j}, \ldots, x_{k}^{j} \in M$. Then there is some $i_{j}$ such that $\alpha_{1 s}^{i_{j}} \neq 0$ for some $s$ in $\Phi_{i_{j}}^{j}$. Hence $\Phi_{i_{j}}^{j}$ contains a basic commutator $a \wedge x_{s}^{j}$ if we rewrite $\Phi_{i_{j}}^{j}$ with respect to a basis $Y Z_{1}^{+} X_{j}^{+} Z_{j}^{-}$where $Z_{1}^{+}=X_{1}^{+} \operatorname{modulo}\langle a\rangle$ and $Z_{j}^{-}=X_{j}^{-}$modulo $\langle a\rangle$. Hence $\Phi_{i_{j}}^{j} \notin N\left(Y X_{1}^{+} X_{2}^{+} X_{2}^{-} \ldots X_{j-1}^{+} X_{j-1}^{-}\right)+N(M)$. Then $\Phi_{i_{2}}^{2}$ and $\Phi_{i_{3}}^{3}$ are linearly independent over $N(M)$. Hence $h \leq 2$, as desired.

Let $F_{2}(p, \kappa)$ be the free group in the variety of all nilpotent groups of class two and with exponent $p$ that has $\kappa$-many free generators. Let $F(3)$ be the $\mathcal{S}$-structure that corresponds to $F_{2}(p, 3)$.

Corollary 7.2. There are infinitely many finite extensions $B_{i}$ of $A=F(3)$ such that $B_{i}$ satisfies $(\Sigma 3)$ and $(\Sigma 4), A$ is selfsufficient in $B_{i}$, and $\delta(A)=\delta\left(B_{i}\right)=3$.

Proof. Let $a_{1}, a_{2}, a_{3}$ be three free generators of $A$. Define $B_{1}$ as $M^{+}$in Lemma 7.1 using the relation $\left[a_{3}, x\right]=\left[a_{1}, a_{2}\right]$. Let $a_{4}$ be the solution in $B_{1}$. To get $B_{2}$ we use $B_{1}$ and $\left[a_{4}, x\right]=\left[a_{2}, a_{3}\right]$. Let $a_{5}$ be the solution in $B_{2}$. In general $B_{i}$ is constructed from $B_{i-1}$ and $\left[a_{i+2}, x\right]=\left[a_{i}, a_{i+1}\right]$ with a solution $a_{i+3}$. The assertion follows from Lemma 7.1. 
Note. Let $G$ be any group that satisfies $(\Sigma 1),(\Sigma 2)^{w}$, and $(\Sigma 3)$. If $A$ is a subspace of $\bar{G}$ of linear dimension 3, then $A$ is selfsufficient in $\bar{G}$ and $A$ generates a subgroup isomorphic to $F_{2}(p, 3)$.

Lemma 7.3. $\Sigma$ is consistent.

Proof. We construct an $\mathcal{S}$-structure $M$ that fulfills $(\Sigma 3),(\Sigma 4)$, and $(\Sigma 5)$. Let $G$ be the corresponding group in $\mathcal{G}$. First we show that $G$ fulfills $(\Sigma 2)$. We assume that $(\Sigma 2)$ is not true in $G$. Then there are $g$ in $M$ and $b$ in $\wedge^{2} M$ such that $g \wedge x=b$ has no solution in $M$. Let $A$ be a finite selfsufficient substructure of $M$ that contains $g$ and $b$. Of course $A$ fulfills $(\Sigma 3)$ and $(\Sigma 4)$. By Lemma 7.1 there is a finite minimal extension $B$ of $A$ such that $B$ fulfills $(\Sigma 3)$ and $(\Sigma 4)$, and there exists some $a$ in $B$ with $g \wedge a=b$. By axiom $(\Sigma 5)$ we can find $B$ and therefore $a$ in $M$, a contradiction to the assumption.

Let $(\Sigma 5)^{\prime}$ be the following consequence of $(\Sigma 5)$ in an $\omega$-saturated model $G$ of $\Sigma$. Let $M$ be the corresponding $\mathcal{S}$-structure:

$(\Sigma 5)^{\prime}$ : Let $A$ and $B$ be finite $\mathcal{S}$-structures that fulfill $(\Sigma 3)$ and $(\Sigma 4)$. Let $A$ be selfsufficient in $B$. If $A$ is selfsufficient in $M$, then there exists a selfsufficient embedding of $B$ in $M$ that extends the embedding of $A$ in $M$.

Note that $(\Sigma 5)^{\prime}$ is not elementary. Next we show that every $M$ that satisfies ( $\Sigma 3),(\Sigma 4)$, and $(\Sigma 5)^{\prime}$ fulfills $(\Sigma 5)$ also. To prove $(\Sigma 5)$ let $A$ be selfsufficient in $B$. Furthermore let $A$ be a $(\operatorname{dim}(B / A)+n)$-selfsufficient substructure of $M$, where $n \geq 2(2 \operatorname{dim}(B)+1)^{2}$. Let $C$ be the finite subspace $C S S(A)$ of $M$. By Theorem 6.9 there is an amalgam $D$ of $B$ and $C$ over $A$ such that $C$ is selfsufficient in $D$ and $B$ is $n$-selfsufficient in $D$. If $D$ plays the role of $B$ and $C$ the role of $A$ in $(\Sigma 5)^{\prime}$, then we have a selfsufficient embedding of $D$ in $M$, that extends the embedding of $C$ in $M$. This gives the desired embedding of $B$ in $M$ with $n$-selfsufficiency.

To get the desired model $G$ of $\Sigma$ we show that there is an $\mathcal{S}$-structure $M$ with the properties $(\Sigma 3),(\Sigma 4)$ and $(\Sigma 5)^{\prime}$. We construct $M$ by successive amalgamation (Theorem 6.9). This is the usual procedure. Let $\left\{A_{i} \subset B_{i}: i<\omega\right\}$ be an enumeration of all pairs of finite structures in $\mathcal{S}$ with $(\Sigma 3)$ and $(\Sigma 4)$ such that $A_{i}$ is selfsufficient in $B_{i}$, and $\operatorname{dim}\left(B_{i}\right) \leq \operatorname{dim}\left(B_{j}\right)$ for $i<j$. By Corollary 7.2 there are infinitely many such pairs. Note that in any $M$ with $(\Sigma 3)$ every substructure generated by three elements is selfsufficient . Let $C_{0}$ be $F(3)$. If $C_{j-1}$ is constructed with $(\Sigma 3)$ and $(\Sigma 4)$, then consider all pairs $A_{i} \subset B_{i}$ with $\operatorname{dim}\left(B_{i}\right) \leq j+2$. Define $D_{0}=C_{j-1}$. If $D_{i-1}$ is constructed with the properties $(\Sigma 3),(\Sigma 4)$, and $C_{j-1}$ is selfsufficient in $D_{i-1}$, then $D_{i}$ is defined in the following way. Let $A_{i}^{1}, \ldots, A_{i}^{s}$ be a list of all copies of $A_{i}$ that are selfsufficient in $C_{j-1}$. They are also selfsufficient in $D_{i-1}$. We apply Theorem 6.9 and obtain the amalgam $D_{i}^{1}$ of $D_{i-1}$ and $B_{i}$ over $A_{i}^{1}$ that again fulfills $(\Sigma 3)$ and $(\Sigma 4) . D_{i-1}$ is selfsufficient in $D_{i}^{1}$. The selfsufficient image $A_{i}^{1}$ of $A_{i}$ in $D_{i-1}$ can be extended to a selfsufficient embedding of $B_{i}$ into $D_{i}^{1}$. In the next step we apply Theorem 6.9 to $D_{i}^{1}$ and $B_{i}$ over $A_{i}^{2}$. We get $D_{i}^{2}$ with $(\Sigma 3)$ and $(\Sigma 4)$ such that $D_{i}^{1}$ is selfsufficient in $D_{i}^{2}$ and there is a selfsufficient embedding of $B_{i}$ in $D_{i}^{2}$ over $A_{i}^{2}$. We iterate the argument and obtain $D_{i}$ as $D_{i}^{s}$. Then we start to work with $A_{i+1} \subset B_{i+1}$, if $\operatorname{dim}\left(B_{i+1}\right) \leq j+2$. If we continue in this way for all pairs $A_{l} \subset B_{l}$ with $\operatorname{dim}\left(B_{l}\right) \leq j+2$, then we obtain $C_{j}$. It is finite and has the properties $(\Sigma 3)$ and $(\Sigma 4)$. $C_{j-1}$ is selfsufficient in $C_{j}$. For every pair $A_{i} \subset B_{i}$ with $\operatorname{dim}\left(B_{i}\right) \leq j+2$ and for every selfsufficient copy of $A_{i}$ in $C_{j-1}$ there is a selfsufficient embedding of $B_{i}$ in $C_{j}$ that continues the embedding of $A_{i}$. Then 
$\bigcup_{j<\omega} C_{j}$ is the desired $\mathcal{S}$-structure such that the corresponding group is a model of $\Sigma$.

Lemma 7.4. $\Sigma$ is complete.

Proof. Let $G_{1}$ and $G_{2}$ be two $\omega$-saturated models of $\Sigma$. Let $M_{i}$ be the $\mathcal{S}$-structure corresponding to $G_{i}(i=1,2)$. We use $a_{i}$ and $b_{i}$ to denote sequences in $G_{1}$ and $G_{2}$ respectively. We assume that the elements of these sequences are linearly independent over the center. Let $\overline{a_{i}}$ and $\overline{b_{i}}$ be the corresponding sequences in $M_{1}$ and $M_{2}$ respectively. We show that player II has a winning strategy in the infinitely long Ehrenfeucht-Fraïssé game. Let $a_{0}$ be a sequence that contains one element $c_{0}$ of $G_{1} \backslash Z\left(G_{1}\right)$, and $b_{0}$ a sequence that contains one element $d_{0}$ in $G_{2} \backslash Z\left(G_{2}\right)$. $\left\langle a_{0}\right\rangle$ and $\left\langle b_{0}\right\rangle$ are selfsufficient in $G_{1}$ and $G_{2}$ respectively, and there is an isomorphism $f_{0}$ of these two subgroups with $f_{0}\left(a_{0}\right)=b_{0}$. After the $(i-1)^{t h}$ round there are sequences $a_{i-1}$ in $G_{1}$ and $b_{i-1}$ in $G_{2}$ of linearly independent elements modulo $Z$ and a map $f_{i-1}$ corresponding to the choice of the players that can be extended to an isomorphism of the subgroups generated by $a_{i-1}$ and $b_{i-1}$ respectively. We require furthermore that $\left\langle a_{i-1}\right\rangle$ and $\left\langle b_{i-1}\right\rangle$ are selfsufficient in $G_{1}$ and $G_{2}$ respectively. Note that it is very important that the players can choose all elements linearly independent modulo $Z$. Now we describe the strategy of player II in the $i^{t h}$ round. Assume w.l.o.g. that player I has chosen $c_{i}$ in $G_{1}$. W.l.o.g. $c_{i}$ is not an element of the subgroup generated by $a_{i-1}$. If $c_{i}$ is not linearly independent from $a_{i-1}$ modulo $Z$, then we can assume that $c_{i} \in Z\left(G_{1}\right)$. By axiom $(\Sigma 2)$ there is some $e_{i}$ such that $\left[c_{0}, e_{i}\right]=c_{i}$. Then $e_{i}$ is linearly independent from $a_{i-1}$ modulo $Z$, since otherwise $c_{i}$ is in the subgroup generated by $a_{i-1}$. In this case player II can assume that $e_{i}$ was chosen. So w.l.o.g. we can suppose that $c_{i}$ is linearly independent from $a_{i-1}$ modulo $Z$. Let $a_{i}$ be a sequence of elements linearly independent modulo $Z$ extending $a_{i-1} c_{i}$ such that its image in $\bar{G}$ is a basis of $C S S\left(\left\langle\bar{a}_{i-1} \bar{c}_{i}\right\rangle\right)$. Player II considers $\left\langle a_{i}\right\rangle \backslash\left\langle a_{i-1}\right\rangle$ as the sequence of new elements chosen by player I. He applies axiom $(\Sigma 5)$ to $M_{2}$, where $\left\langle\overline{a_{i-1}}\right\rangle$ plays the role of $A$ and $\left\langle\overline{a_{i}}\right\rangle$ plays the role of $B$. For every sufficiently large $n$ he gets an $n$-selfsufficient embedding $\overline{f_{i}}$ of $\left\langle\overline{a_{i}}\right\rangle$ into $M_{2}$, where $\overline{f_{i}}$ extends the isomorphism of $\left\langle\overline{a_{i-1}}\right\rangle$ onto $\left\langle\overline{b_{i-1}}\right\rangle$ induced by $f_{i-1}$. By Lemma 3.2 we can lift $\overline{f_{i}}$ to an isomorphism $f_{i}$ of $\left\langle a_{i}\right\rangle$ into $G_{2}$ that extends $f_{i-1}$. By the $\omega$-saturation of $G_{2}$ we can find $f_{i}$ in such a way that $\left\langle f_{i}\left(a_{i}\right)\right\rangle$ is selfsufficient in $G_{2}$. We have the desired properties if we denote $f\left(a_{i}\right)$ by $b_{i}$ in an appropriate way.

If $\left\langle a_{0}\right\rangle$ and $\left\langle b_{0}\right\rangle$ in the proof above are any isomorphic selfsufficient subgroups with $(\Sigma 2)^{w}$, then the same proof works. If $A$ and $B$ are isomorphic selfsufficient subgroups of the same model $G$ of $\Sigma$, then we can extend $G$ to an $\omega$-saturated elementary extension $G_{1}=G_{2}=G^{+}$. Then $A$ and $B$ are selfsufficient in $G^{+}$too. We have:

Lemma 7.5. If $A$ and $B$ are isomorphic finite selfsufficient subgroups of a model of $\Sigma$ that satisfy $(\Sigma 2)^{w}$, then they have the same elementary type over the empty set.

To prove the uncountable categoricity of $\Sigma$ we show that for every model $G$ the geometrical closure $c l$ and the algebraic closure $a c l$ coincide in $\bar{G}$. It was to ensure this that the axiom $(\Sigma 4)$ was introduced and the Second Amalgamation Theorem was proved. 
Lemma 7.6. Let $G$ be a model of $\Sigma$. Let $A$ be a finite subgroup of $G$ with the property $(\Sigma 2)^{w}$. Let $\bar{A}$ be its image in $\bar{G}$.

(i) For all $a$ in $\bar{G}$ we have $a \in \operatorname{acl}(\bar{A})$ if and only if $a \in \operatorname{cl}(\bar{A})$.

(ii) For all elements $a$ and $b$ in $G$ whose images in $\bar{G}$ are not in acl $(A)$, we obtain $t(a / A)=t(b / A)$.

Proof. Since $C S S(\bar{A}) \subseteq \operatorname{acl}(\bar{A})$ we can assume that $A$ is selfsufficient in $G$.

$\operatorname{ad}(\mathrm{i})$. $(\leftarrow)$. There is a non-refinable tower $\bar{A}=\bar{B}_{0} \subset \bar{B}_{1} \subset \bar{B}_{2} \ldots \subset \bar{B}_{n}=$ $C S S(\langle\bar{A}\{a\}\rangle)$ such that $\bar{B}_{i+1}$ is a minimal extension of $\bar{B}_{i}$ for all $0 \leq i \leq n-1$. It is sufficient to show that $\bar{B}_{i+1} \subseteq \operatorname{acl}\left(\bar{B}_{i}\right)$ for all these $i$. Otherwise there are infinitely many realizations of the minimal extension $\bar{B}_{i+1}$ over $\bar{B}_{i}$. Note that all $\bar{B}_{i}$ are selfsufficient in $\bar{G}$. Therefore by Lemma 5.3 there are infinitely many realizations of the minimal extension $\bar{B}_{i+1}$ over $\bar{B}_{i}$, that are in free composition over $\bar{B}_{i}$. This is impossible by $(\Sigma 4)$, as mentioned in Lemma 6.24$)$.

$(\rightarrow)$. If $a \notin \operatorname{cl}(\bar{A})$, then $d(\langle\bar{A}\{a\}\rangle)>d(\bar{A})$ and by Lemma $3.8 d(\langle\bar{A}\{a\}\rangle)=$ $d(\bar{A})+1$. Since $\bar{A}$ is selfsufficient in $\bar{G}$, we have that $\langle\bar{A}\{a\}\rangle$ is selfsufficient in $\bar{G}$. If $d(\bar{A}) \leq 2$, then every $c \notin \bar{A}$ is isomorphic to $a$ over $\bar{A}$ with respect to the full group structure. By Lemma 7.5, $t(a / A)=t(c / A)$. Therefore $a \notin a c l(\bar{A})$. If $d(\bar{A}) \geq 3$, then by Corollary $7.2 \operatorname{cl}(\bar{A})$ is infinite. For each $c \in \operatorname{cl}(\bar{A})$ we have $d(\langle\bar{A}\{a+c\}\rangle)=d(\bar{A})+1$. Then $a+c$ is isomorphic to $a$ over $\bar{A}$ with respect to the full group structure, and $\langle\bar{A}\{a+c\}\rangle$ and $\langle\bar{A}\{a\}\rangle$ are selfsufficient in $\bar{G}$. By Lemma 7.5 again $t(a+c / A)=t(a / A)$ for infinitely many $c$. We obtain $a \notin \operatorname{acl}(\bar{A})$, as desired.

ad(ii). As above we have $A$ selfsufficient in $G$. By (i), $d(\langle\bar{A}\{a\}\rangle)=d(\bar{A})+1$ and $d(\langle\bar{A}\{b\}\rangle)=d(\bar{A})+1$. It follows that $a$ and $b$ are isomorphic over $A$. By Lemma 7.5 we obtain the assertion.

Theorem 7.7. $\Sigma$ is uncountably categorical. If $G$ is a model of $\Sigma$, then $G / Z(G)$ and $Z(G)$ are strongly minimal. $\Sigma$ has Morley rank 2. $\Sigma$ is not countably categorical. $\Sigma$ has a non-locally-modular geometry.

Proof. We consider $\Sigma$. By Lemmas 7.3 and $7.4, \Sigma$ is consistent and complete. By Corollary 7.2 and Lemma 7.6, $\operatorname{acl}(\{a, b, c\})$ is infinite for elements $a, b, c$ that are linearly independent over $Z(G)$. It follows that $\Sigma$ is not countably categorical. To show the uncountable categoricity let $\mathrm{G}$ be any model of $\Sigma$ embedded in the monster model $\mathbf{C}$. Let $a \in \mathbf{C} \backslash G$. If $a h^{-1} \in Z(\mathbf{C})$ for some $h \in G$ and $g$ is any element of $G$ not in $Z(G)$, then by $(\Sigma 2)$ there exists $c \in \mathbf{C} \backslash G+Z(\mathbf{C})$ such that $a h^{-1}=[g, c]$. Therefore every $t(a / G)$ with a formula $Z\left(x h^{-1}\right)$ is algebraic over a type $t(c / G)$ with $\neg Z\left(x h^{-1}\right)$ for all $h \in G$. By Lemma 7.6 there exists only one nonalgebraic type $t(a / G)$ with $\neg Z\left(x h^{-1}\right)$ for all $h \in G$. The uncountable categoricity of $T$ follows. Furthermore, $G / Z(G)$ is strongly minimal by Lemma 7.6. By $(\Sigma 2)$ and ( $\Sigma 3)$, for any $g \in G \backslash Z(G)$ the map $[g, x]$ is one-to-one from $G /\langle Z(G) \cup\{g\}\rangle$ onto $Z(G)$. Hence $Z(G)$ is strongly minimal. Since for uncountably categorical theories Morley rank and Lascar rank coincide, we obtain Morley rank 2 for $\Sigma$ by the Berline-Lascar inequalities for the Lascar rank of groups. By [13] $\Sigma$ has a non-locally-modular geometry, since the models of $\Sigma$ are not Abelian by finite. It is easy to prove this directly. 


\section{CM-triviality}

Let $\Sigma$ be the theory constructed above. In this section we prove the noninterpretability of a field in $\Sigma$. The first proof of this result was based on the investigation of the group-configuration of a group interpreted in $\Sigma$. It was shown that such a group has to be nilpotent by finite and of bounded exponent. (See [6], section 7.) Here we prove CM-triviality of $\Sigma$, following a suggestion of E. Hrushovski and A. Pillay. This geometrical property of a stable theory implies the non-interpretability of a field. Hence $\Sigma$ does not allow the interpretation of a field, as desired. Furthermore, Pillay [21] has shown that it is impossible to interpret a simple group in an $\omega$-stable theory of finite Morley rank with CM-triviality. Hence no simple group is interpretable in $\Sigma$. By Theorem 2.1 we also obtain that every group interpretable in $\Sigma$ is nilpotent by finite, and of bounded exponent up to an Abelian direct summand.

CM-triviality was introduced by E. Hrushovski in [12]. A stable theory T is CM-trivial if the following three equivalent conditions concerning $T^{e q}$ are fulfilled.

(CMT1): Suppose that $B_{1}, B_{2}$, and $E$ are algebraically closed, $B_{1}$ and $B_{2}$ are independent over $E$, $\operatorname{acl}\left(B_{1} B_{2}\right) \cap \operatorname{acl}\left(E B_{i}\right)=B_{i}$, and $B_{i} \cap E=A$. Then $B_{1}$ and $B_{2}$ are independent over $A$.

(CMT2): If $E$ is algebraically closed and $C_{1}$ and $C_{2}$ are independent over $E$, then $C_{1}$ and $C_{2}$ are independent over $\operatorname{acl}\left(C_{1} C_{2}\right) \cap E$.

$(C M T 3)$ : Let $A$ and $B$ be algebraically closed. Assume $\operatorname{acl}(A c) \cap B=A$. Then $C b(c / A) \subseteq \operatorname{acl}(C b(c / B))$.

A 1-based theory is CM-trivial. E. Hrushovski's new strongly minimal theory is CM-trivial but not 1-based. (For all of the above see [12].) If $T_{c}$ is the theory of a model of $T$ with constants for some fixed finite sequence $c$, then $T$ is CM-trivial iff $T_{c}$ is CM-trivial, as mentioned by A. Pillay in [21]. Furthermore he mentioned in that paper that it is sufficient to replace $(C M T 3)$ by the following:

(CMT3): Let $c$ be a tuple in the monster model $\mathbf{C}$ of $T$. Assume that $M \preceq N$ are elementary submodels of $\mathbf{C}$ with $\operatorname{acl}(c M) \cap N=M$. Then $C b(t p(c / M)) \subseteq$ $\operatorname{acl}(C b(t p(c / N)))$.

Let $G$ be any model of $\Sigma$. Let $a_{1}, a_{2}$, and $a_{3}$ be three elements in $G$ that are linearly independent over $Z(G)$. Let $\Sigma^{*}$ be the elementary theory of $\left(G ; a_{1}, a_{2}, a_{3}\right)$ with constants for the three fixed elements. Note that $\Sigma^{*}$ is independent from the choice of $G$ and the elements $a_{1}, a_{2}, a_{3}$, because of axiom ( $\left.\Sigma 3\right)$ and Lemma 7.5. Let $\overline{\Sigma^{*}}$ be the elementary theory of the vector space $\bar{G}$ with the fixed elements $a_{1} / Z$, $a_{2} / Z, a_{3} / Z$ and the relation $R(x, y, z)$ defined by $\left[a_{1} / Z, x\right]=[y, z]$, where $[$,$] is$ the alternating bilinear map on $\bar{G} \times \bar{G}$ into $Z(G)$ given by the commutator. Let $\Gamma$ be this interpretation of $\overline{\Sigma^{*}}$ in $\Sigma^{*}$.

Lemma 8.1. There is an interpretation $\Lambda$ of $\Sigma^{*}$ in $\overline{\Sigma^{*}}$ such that every model $\left(G ; a_{1}, a_{2}, a_{3}\right)$ of $\Sigma^{*}$ is isomorphic to $\Lambda\left(\Gamma\left(G ; a_{1}, a_{2}, a_{3}\right)\right)$ and for every model $M$ of $\overline{\Sigma^{*}}$ there is an isomorphism between $M$ and $\Gamma(\Lambda(M))$ definable in $M^{e q}$.

Proof. Let $\left(G ; a_{1}, a_{2}, a_{3}\right)$ be a model of $\Sigma^{*}$ and let $B_{G}$ be the corresponding alternating bilinear map in $\mathcal{B}$ of $\bar{G} \times \bar{G}$ into $Z(G)$ with three fixed constants. By $(\Sigma 2)$ and $(\Sigma 3),\left[a_{1} / Z, x\right]$ defines a vector space isomorphism of $\bar{G} /\left\langle a_{1} / Z\right\rangle$ onto $Z(G)$. Hence $R(x, y, z)$ gives an interpretation of $B_{G}$ in $\Gamma(G)$. To prove the assertion we define an interpretation of $G$ in $B_{G}$. We write the vector spaces $\bar{G}$ and $Z(G)$ 
additively as above. Starting with $B_{G}$ we define in $B_{G}$ a group $G^{+}$isomorphic to $G$. $G^{+}$consists of all tuples $(a, x)$ with $a \in \bar{G}$ and $x \in Z(G)$. The multiplication is given by $(a, x) \circ(b, y)=(a+b, x+y+[a, b])$. It is easily seen that this defines a group. $(0,0)$ is the unit and $(-a,-x)$ is the inverse element of $(a, x)$. Furthermore $[(a, x),(b, y)]=(0,2[a, b]) . G^{+}$fulfills $(\Sigma 1)$ and $(\Sigma 2)^{w}$. If $g_{0}, g_{1}, g_{2}, \ldots$ is a basis of $\bar{G}$, then the images of $\left(g_{0}, 0\right),\left(g_{1}, 0\right),\left(g_{2}, 0\right), \ldots$ in $\bar{G}^{+}$form a basis of $\bar{G}^{+}$. A linear combination of commutators over $g_{0}, g_{1}, g_{2}, \ldots$ is 0 if and only if the corresponding linear combination of commutators over $\left(g_{0}, 0\right),\left(g_{1}, 0\right),\left(g_{2}, 0\right), \ldots$ is 0 . By Lemma $3.1 G$ and $G^{+}$are isomorphic, as desired. The definition of $G^{+}$in $\Gamma(G)$ gives the desired interpretation $\Lambda$.

By Lemma 8.1 we have that $\overline{\Sigma^{*}}$ is the theory of the full structure definable on $\bar{G}$ in $\left(G ; a_{1}, a_{2}, a_{3}\right)$.

Now we consider a model $\Gamma(G)$ of $\overline{\Sigma^{*}}$ living in a model $G$ of $\Sigma^{*}$ via the given interpretation. We do not mention the fixed three elements, but we will need them later. We get that $\overline{\Sigma^{*}}$ is strongly minimal. Furthermore an automorphism $\chi$ of $\Gamma(G)$ corresponds to an automorphism of the alternating bilinear map $B_{G}$ living in $G$. By Lemma 3.1 we can extend $\chi$ to an automorphism of $G$. Hence $\Gamma$ is an interpretation without new information in the sense of [3]. As shown in [4], forking for subsets of $\Gamma(G)$ does not depend on whether we consider them in $\overline{\Sigma^{*}}$ or in $\Sigma^{*}$.

Since we have fixed three linearly independent elements in every model $M$ of $\overline{\Sigma^{*}}, \operatorname{acl}(\emptyset)$ is infinite in $M$ (in the real sort!). As mentioned by A. Pillay in [26], a strongly minimal theory with this property allows weak elimination of imaginaries. This notion was introduced by B. Poizat in [25]. We will use this property to prove CM-triviality of $\overline{\Sigma^{*}}$. First we will show that CM-triviality of $\overline{\Sigma^{*}}$ will imply CM-triviality of $\Sigma$. For this we will use the third form of the definition.

Lemma 8.2. If $\overline{\Sigma^{*}}$ is CM-trivial, then $\Sigma$ is CM-trivial.

Proof. Assume that $\overline{\Sigma^{*}}$ is CM-trivial. We will show the CM-triviality of $\Sigma^{*}$. Then the $C M$-triviality of $\Sigma$ follows, as mentioned above (see [21]). We use (CMT3). Assume that $c$ is a tuple in the monster model $\mathbf{C}$ of $\Sigma^{*}$. Let $G \preceq H$ be elementary submodels of $\mathbf{C}$ such that $\operatorname{acl}(c G) \cap H=G$. We have to show that $C b(t p(c / G)) \subseteq$ $C b(t p(c / H))$. Since in a model of $\Sigma^{*}$ every tuple is interalgebraic with a tuple of elements that are linearly independent over $Z+\left\langle\left\{a_{1}, a_{2}, a_{3}\right\}\right\rangle$, we can assume that the elements of $c$ are linearly independent over $Z+\left\langle\left\{a_{1}, a_{2}, a_{3}\right\}\right\rangle$. Let us write $\Gamma(c)$ for the image of $c$ in the monster model $\boldsymbol{\Gamma}(\mathbf{C})$ of $\Sigma^{*}$. Let $\Gamma(G)$ and $\Gamma(H)$ be the elementary submodels of $\boldsymbol{\Gamma}(\mathbf{C})$ that correspond to $G$ and $H$ respectively. Again $\Gamma(G) \preceq \Gamma(H)$, and in $\boldsymbol{\Gamma}(\mathbf{C})$ we have $\operatorname{acl}(\Gamma(c) \Gamma(G)) \cap \Gamma(H)=\Gamma(G)$. (CMT3) for $\overline{\Sigma^{*}}$ implies $C b(t p(\Gamma(c) / \Gamma(G))) \subseteq \operatorname{acl}(C b(t p(\Gamma(c) / \Gamma(H))))$ By the weak elimination of imaginaries for $\overline{\Sigma^{*}}$ we have finite subspaces $A_{0} \subseteq A_{1}$ of $\boldsymbol{\Gamma}(\mathbf{C})$ such that $A_{0} \subseteq \Gamma(G)$ is interalgebraic with $C b(t p(\Gamma(c) / \Gamma(G)))$ and $A_{1} \subseteq \Gamma(H)$ is interalgebraic with $C b(t p(\Gamma(c) / \Gamma(H)))$. Now we consider $A_{0}$ and $A_{1}$ as subspaces in the sort $\mathbf{C} / Z(C)$ in C. We claim that $A_{0}$ is interalgebraic with $C b(t p(c / G))$ and $A_{1}$ is interalgebraic with $C b(t p(c / H))$. Then the assertion of the lemma follows. We consider the first case. The second works in the same way.

Let $K$ be any elementary extension of $G$ and let $t p(d / K)$ be the non-forking extension of $t p(c / G)$. We have to show that for all possible $K$ and $d$ above:

(i) Let $\Pi$ be the set of all automorphisms of the monster model $\mathbf{C}$ that fixes $A_{0}$ pointwise and $K$ setwise. Then $\{t p(\pi(d) / K): \pi \in \Pi\}$ is finite. 
(ii) Let $\Xi$ be the set of all automorphisms $\xi$ of the monster model $\mathbf{C}$ such that $t p(\xi(d) / K)=t p(d / K)$ and $\xi$ fixes $K$ setwise. Then $\left\{\xi\left(A_{0}\right): \xi \in \Xi\right\}$ is finite.

First we prove (i). If we restrict all $\pi \in \Pi$ to $\boldsymbol{\Gamma}(\mathbf{C})$, then $\{t p(\pi(\Gamma(d)) / \Gamma(K))$ : $\pi \in \Pi\}$ is finite since $C b(t p(\Gamma(c) / \Gamma(G))) \subseteq \operatorname{acl}\left(A_{0}\right)$. Assume $\pi_{1}, \pi_{2} \in \Pi$ and $\operatorname{tp}\left(\pi_{1}(\Gamma(d)) / \Gamma(K)\right)=t p\left(\pi_{2}(\Gamma(d)) / \Gamma(K)\right)$. It remains to show that $t p\left(\pi_{1}(d) / K\right)=$ $t p\left(\pi_{2}(d) / K\right)$. Let $B$ be any finite subgroup of $K$ with $(\Sigma 2)^{w}$ that contains $a_{1}, a_{2}$, and $a_{3}$. In $\boldsymbol{\Gamma}(\mathbf{C})$ we have an isomorphism $\eta$ of

$$
C S S\left(\left\langle\Gamma\left(\pi_{1}(d)\right)\right\rangle+\Gamma(B)\right) \text { onto } C S S\left(\left\langle\Gamma\left(\pi_{2}(d)\right)\right\rangle+\Gamma(B)\right)
$$

that is the identity on

$$
C S S\left(\left\langle\Gamma\left(\pi_{1}(d)\right)\right\rangle+\Gamma(B)\right) \cap \Gamma(K)=C S S\left(\left\langle\Gamma\left(\pi_{2}(d)\right)\right\rangle+\Gamma(B)\right) \cap \Gamma(K)
$$

and that fulfills $\eta\left(\pi_{1}(d)\right)=\pi_{2}(d)$. By Lemma 7.5 we get

$$
t p\left(\pi_{1}(d) / K\right)=t p\left(\pi_{2}(d) / K\right) \text {, }
$$

as desired.

(ii) follows immediately from the corresponding statement in $\mathbf{\Gamma}(\mathbf{C})$.

The following lemma is an essential part of the proof of the CM-triviality of $\overline{\Sigma^{*}}$.

Lemma 8.3. Let $A$ and $B$ be finite $\mathcal{S}$-structures that satisfy $(\Sigma 3)$, where $B$ is a minimal extension of $A$. Assume that $b_{1}, \ldots, b_{n}$ is a linear basis of $B$ over $A$, and that $N(B)$ is freely generated over $N(A)$ by relations $\Delta_{1}, \ldots, \Delta_{n}$ of the following form:

$$
\Delta_{i}=\sum_{1 \leq j \leq n} a_{j}^{i} \wedge b_{j}+\Psi_{i}
$$

where $a_{j}^{i} \in A$ and $\Psi_{i} \in \wedge^{2} A$. Finally, assume that it is impossible to present $N(B)$ over $N(A)$ as above without the use of nontrivial $\Psi_{i}$ 's. Then ( $\left.\Sigma 4\right)$ for A implies ( $\Sigma 4)$ for $B$.

Note first that we can replace $b_{1}, \ldots, b_{n}$ by any other basis of $B$ over $A$. The basis of $N(B)$ over $N(A)$ we get with respect to the new basis of $B$ over $A$ has again the form described above and the new elements replacing the $a_{j}^{i}$, s generate again the subspace $\left\langle\left\{a_{j}^{i}: 1 \leq i, j \leq n\right\}\right\rangle$ of $A$. Hence this subspace is uniquely determined by $B$.

Proof. Let $Y X_{1}^{+} X_{2}^{+} X_{2}^{-} \ldots X_{h}^{+} X_{h}^{-}$be a reduced $k$-system in $B$. If it is completely in $A$, then we are done. Otherwise we choose a basis $Y^{a} Y^{b}$ of $\langle Y\rangle$ such that $\left\langle Y^{a}\right\rangle=$ $\langle Y\rangle \cap A$. If there is some $X_{i}^{+}$such that $X_{i}^{+} \subseteq A$ modulo $\langle Y\rangle$, then w.l.o.g. we can assume that $i=1$. Use (S3) and (S4). Using a well-tolerated automorphism we can assume that $X_{1}^{+}=X^{a} X^{b}$, where $Y^{a} X^{a}$ is a basis of $\left\langle Y X_{1}^{+}\right\rangle \cap A$. For $2 \leq i \leq h$ we choose a basis $V_{i} U_{i}$ of $\left\langle Y X_{1}^{+} X_{2}^{+} X_{2}^{-} \ldots X_{i}^{+} X_{i}^{-}\right\rangle$over $\left\langle Y X_{1}^{+} X_{2}^{+} X_{2}^{-} \ldots X_{i-1}^{+} X_{i-1}^{-}\right\rangle$ such that $V_{i} \subseteq A$ and $U_{i}$ is linearly independent over $\left\langle Y X_{1}^{+} X_{2}^{+} X_{2}^{-} \ldots X_{i-1}^{+} X_{i-1}^{-}\right\rangle+$ $A$. To prove the next claim we use the special form of the free generators of $N(B)$ over $N(A)$.

(1) Assume $k>0$. Then for $2 \leq i \leq h V_{i} \neq \emptyset$.

Proof of (1). Assume $V_{i}=\emptyset$. We consider the condition (S1). Then $X_{i}^{+} X_{i}^{-}$is part of a basis of $B$ over $A+\left\langle Y X_{1}^{+} X_{2}^{+} X_{2}^{-} \ldots X_{i-1}^{+} X_{i-1}^{-}\right\rangle$. Since $k>0$ there are $1 \leq s<t \leq k$ such that $\alpha_{s t}^{l}\left(x_{s}^{i} \wedge x_{t}^{i}\right)$ occurs in $\Phi_{l}^{i}$ in (S1), where $\alpha_{s t}^{l} \neq 0$. But 
then it is impossible to get $\Phi_{l}^{i}$ as a linear combination of the $\Delta_{j}$ 's above modulo $\wedge^{2} A$.

(2) Assume $V_{i} \neq \emptyset$ for $2 \leq i \leq h$. Then $\left\langle Y X_{1}^{+}\right\rangle \nsubseteq A$.

Proof of (2). We assume toward a contradiction that $\left\langle Y X_{1}^{+}\right\rangle \subseteq A$. Since the reduced $k$-sequence under consideration is not contained in $A$, there exists a minimal $i_{0}$ such that $2 \leq i_{0} \leq h$ and $U_{i_{0}} \neq \emptyset$. Since $\left\langle Y X_{1}^{+} X_{2}^{+} X_{2}^{-} \ldots X_{i_{0}}^{+} X_{i_{0}}^{-}\right\rangle$is a minimal extension of $\left\langle Y X_{1}^{+} X_{2}^{+} X_{2}^{-} \ldots X_{i_{0}-1}^{+} X_{i_{0}-1}^{-}\right\rangle, V_{i_{0}} \neq \emptyset$, and $U_{i_{0}} \neq \emptyset$, we get

$$
\begin{aligned}
& \operatorname{dim}\left(N\left(\left\langle Y X_{1}^{+} X_{2}^{+} X_{2}^{-} \ldots X_{i_{0}}^{+} X_{i_{0}}^{-}\right\rangle\right)\right) \\
& \quad-\operatorname{dim}\left(N\left(\left\langle Y X_{1}^{+} X_{2}^{+} X_{2}^{-} \ldots X_{i_{0}-1}^{+} X_{i_{0}-1}^{-} V_{i_{0}}\right)\right)>\left|U_{i_{0}}\right| .\right.
\end{aligned}
$$

But then $\delta\left(A+\left\langle U_{i_{0}}\right\rangle\right)<\delta(A)$, a contradiction to the minimality of $B$ over $A$.

The next assertion together with (1) implies the assertion of the lemma in the case $0<k$.

(3) Assume that $V_{i} \neq \emptyset$ for $2 \leq i \leq h$. Then $h<|Y|+k+2$, as desired.

Proof of (3). Assume $|Y|+k+2 \leq h$. Let $n_{i}=\left|U_{i}\right|$. We show by induction on $i$ (for $2 \leq i \leq h$ ) that:

$\left(*_{i}\right)$ There are $m_{i}$ relations $\Theta_{1}, \ldots, \Theta_{m_{i}}$ in $N\left(Y X_{1}^{+} X_{2}^{+} X_{2}^{-} \ldots X_{i}^{+} X_{i}^{-}\right)$linearly independent over $N(A)$, where $m_{i}=\sum_{2 \leq j \leq i}\left(n_{j}+1\right)$.

We prove the assertion for $i+1(1 \leq i<h)$. First assume $U_{i+1} \neq \emptyset$. Since $\left\langle Y X_{1}^{+} X_{2}^{+} X_{2}^{-} \ldots X_{i+1}^{+} X_{i+1}^{-}\right\rangle$is a minimal extension of $\left\langle Y X_{1}^{+} X_{2}^{+} X_{2}^{-} \ldots X_{i}^{+} X_{i}^{-}\right\rangle$and $V_{i+1} \neq \emptyset$, there are $\Theta_{m_{i}+1}, \ldots, \Theta_{m_{i}+n_{i+1}+1} \in N\left(\left\langle Y X_{1}^{+} X_{2}^{+} X_{2}^{-} \ldots X_{i+1}^{+} X_{i+1}^{-}\right\rangle\right)$ which are linearly independent over $N\left(\left\langle Y X_{1}^{+} X_{2}^{+} X_{2}^{-} \ldots X_{i}^{+} X_{i}^{-} V_{i+1}\right\rangle\right)$. Then

$$
\Theta_{m_{i}+1}, \ldots, \Theta_{m_{i}+n_{i+1}+1}
$$

are linearly independent over

$$
N\left(\left\langle Y X_{1}^{+} X_{2}^{+} X_{2}^{-} \ldots X_{i}^{+} X_{i}^{-} V_{i+1}\right\rangle\right)+\wedge^{2} A,
$$

since every nontrivial linear combination of them contains some $u \wedge x$ where $u \in$ $U_{i+1}$. The assertion follows.

If $U_{i+1}=\emptyset$, then by (2) we get again $Y^{b} X^{b} \neq \emptyset$. We will show that we can find the desired $\Theta_{m_{i}+1}$ in

$$
N\left(\left\langle Y X_{1}^{+} X_{2}^{+} X_{2}^{-} \ldots X_{i+1}^{+} X_{i+1}^{-}\right\rangle\right) \backslash \wedge^{2}\left(\left\langle Y X_{1}^{+} X_{2}^{+} X_{2}^{-} \ldots X_{i}^{+} X_{i}^{-}\right\rangle\right)+\wedge^{2} A
$$

We work with the basis $Y^{a} Y^{b} X^{a} X^{b} U_{2} V_{2} \ldots U_{i} V_{i} V_{i+1}$. We consider $\Phi_{j}^{i+1}$ in (S1):

$$
\begin{aligned}
\sum_{1 \leq s<t \leq k} \alpha_{s t}^{j} & \left(\left(x_{s}^{i+1} \wedge x_{t}^{i+1}\right)-\left(x_{s}^{1} \wedge x_{t}^{1}\right)\right) \\
& +\sum_{1 \leq t \leq k}\left(y_{t}^{j} \wedge\left(x_{t}^{i+1}-x_{t}^{1}\right)\right)+\sum_{k<t \leq r}\left(y_{t}^{j} \wedge z_{t}^{i+1}\right) .
\end{aligned}
$$

By assumption $X^{a} X^{b}=X_{1}^{+}$. We can choose $V_{i+1}=\left\{v_{1}, \ldots, v_{r}\right\} \subseteq A$ in such a way that $x_{t}^{i+1}=v_{t}+c_{t}$ for $1 \leq t \leq k$ and $z_{t}^{i+1}=v_{t}+c_{t}$ for $k<t \leq r$, where $c_{i} \in\left\langle Y^{b} X^{b} U_{2} \ldots U_{i}\right\rangle$. First we assume $Y^{b} \neq \emptyset$. Let $y$ be an element of $Y^{b}$. We show that there is some $j$ such that the representation of $\Phi_{j}^{i+1}$ with respect to the basis $Y^{a} Y^{b} X^{a} X^{b} U_{2} V_{2} \ldots U_{i} V_{i} V_{i+1}$ contains a basic commutator $y \wedge v_{t}$. Then $\Phi_{j}^{i+1} \notin$ $\wedge^{2}\left\langle Y X_{1}^{+} X_{2}^{+} X_{2}^{-} \ldots X_{i}^{+} X_{i}^{-}\right\rangle+\wedge^{2} A$ and the assertion follows. First we assume that 
$y$ occurs in the linear presentation of $y_{t}^{j}$ for some $t>k$. Then $y \wedge v_{t}$ cannot vanish. Now we assume that, for all $j, y$ only occurs in the presentation of $y_{t}^{j}$ with respect to the basis $Y=Y^{a} Y^{b}$ if $t \leq k$. Then $y_{t}^{j}=\gamma_{t}^{j} y+w_{t}^{j}$ for $1 \leq j \leq r$ and $1 \leq t \leq k$, where $w_{t}^{j} \in\langle Y \backslash\{y\}\rangle$ and $y_{t}^{j} \in\langle Y \backslash\{y\}\rangle$ if $k<t$. For $1 \leq t \leq k$ we write $x_{t}^{i+1}=v_{t}+\epsilon_{t} y+d_{t}$, where $v_{t} \in V_{i+1}$ as above and $d_{t} \in\left\langle\left(Y^{b} \backslash\{y\}\right) X^{b} U_{2} \ldots U_{i}\right\rangle$. If we rewrite $\Phi_{j}^{i+1}$ with respect to the basis $Y X^{a} X^{b} U_{2} V_{2} \ldots U_{i} V_{i} V_{i+1}$, then the coefficient of the basic commutator $y \wedge v_{t}$ is

$$
\sum_{1 \leq s<t} \alpha_{s t}^{j} \epsilon_{t}-\sum_{t<s \leq k} \alpha_{t s}^{j} \epsilon_{t}+\gamma_{t}^{j}
$$

If there is some $j(1 \leq j \leq r)$ and some $t(1 \leq t \leq k)$ such that this coefficient is not 0 , then $\Phi_{j}^{i+1} \notin \wedge^{2}\left(\left\langle Y X_{1}^{+} X_{2}^{+} X_{2}^{-} \ldots X_{i}^{+} X_{i}^{-}\right\rangle\right)+\wedge^{2} A$, as desired. If all these coefficients are 0 , then we consider the following well-tolerated automorphism $e$ : $e\left(x_{t}^{i}\right)=x_{t}^{i}-\epsilon_{t} y$ for $1 \leq t \leq k, e\left(z_{t}^{i}\right)=z_{t}^{i}$, and $e(a)=a$ for $a \in\langle Y\rangle$. If we rewrite $\Phi_{j}^{i}$ with respect to the basis $e\left(Y X_{1}^{+} X_{2}^{+} X_{2}^{-} \ldots X_{h}^{+} X_{h}^{-}\right)$, then

$$
\begin{aligned}
\Phi_{j}^{i} & =\sum_{1 \leq s<t \leq k} \alpha_{s t}^{j}\left(\left(e\left(x_{s}^{i}\right) \wedge e\left(x_{t}^{i}\right)\right)-\left(e\left(x_{s}^{1}\right) \wedge e\left(x_{t}^{1}\right)\right)\right) \\
& +\sum_{1 \leq t \leq k}\left(w_{t}^{j} \wedge\left(e\left(x_{t}^{i}\right)-e\left(x_{t}^{1}\right)\right)\right)+\sum_{k<t \leq r}\left(y_{t}^{j} \wedge e\left(z_{t}^{i}\right)\right) .
\end{aligned}
$$

Since $y_{t}^{j} \in\langle Y \backslash\{y\}\rangle$ for $k<t \leq h$ and $w_{t}^{j} \in\langle Y \backslash\{y\}\rangle$, we get (S1) with a smaller $Y$, a contradiction to (S4).

If $Y^{b}=\emptyset$, then $X^{b} \neq \emptyset$ by (2). By assumption $X^{b} \neq \emptyset$ implies that $X_{i+1}^{+} \nsubseteq A$ modulo $\langle Y\rangle$. Hence there is some $t_{0}$ such that $1 \leq t_{0} \leq k$ and $c_{t_{0}} \notin\langle Y\rangle$. We fix some $u$ in $X^{b} U_{2}, \ldots, U_{i}$ that occurs in $c_{t_{0}}$. Then we apply a well-tolerated automorphism to $\left\langle X_{i+1}^{+}\right\rangle$such that $u$ occurs exactly in $c_{t_{0}}$. That means we subtract suitable $s x_{t_{0}}^{i+1}$ from each $x_{t}^{i+1}$ with $t \neq t_{0}$ and $1 \leq t \leq k$. Then we choose some $s$ and $j$ with $\alpha_{t_{0} s}^{j} \neq 0$ or $\alpha_{s t_{0}}^{j} \neq 0$ respectively. It follows that $u \wedge v_{s}$ will not vanish in $\Phi_{j}^{i+1}$. Hence $\Phi_{j}^{i+1} \in N\left(\left\langle Y X_{1}^{+} X_{2}^{+} X_{2}^{-} \ldots X_{i+1}^{+} X_{i+1}^{-}\right\rangle\right) \backslash\left(\wedge^{2}\left(\left\langle Y X_{1}^{+} X_{2}^{+} X_{2}^{-} \ldots X_{i}^{+} X_{i}^{-}\right\rangle\right)+\wedge^{2} A\right)$.

Now we compute $\delta\left(Y X_{1}^{+} X_{2}^{+} X_{2}^{-} \ldots X_{h}^{+} X_{h}^{-}+A\right)-\delta(A)$ :

$$
\operatorname{dim}\left(Y X_{1}^{+} X_{2}^{+} X_{2}^{-} \ldots X_{h}^{+} X_{h}^{-}+A\right)-\operatorname{dim}(A)=\left|Y^{b}\right|+\left|X^{b}\right|+\sum_{2 \leq j \leq h} n_{j} .
$$

On the other hand by the induction above

$$
\operatorname{dim}\left(N\left(\left\langle Y X_{1}^{+} X_{2}^{+} X_{2}^{-} \ldots X_{h}^{+} X_{h}^{-}\right\rangle+A\right)\right)-\operatorname{dim}(N(A)) \geq\left(\sum_{2 \leq j \leq h} n_{j}\right)+h-1 .
$$

Hence

$$
\delta\left(\left\langle Y X_{1}^{+} X_{2}^{+} X_{2}^{-} \ldots X_{h}^{+} X_{h}^{-}\right\rangle+A\right)-\delta(A) \leq\left|Y^{b}\right|+\left|X^{b}\right|-h+1 .
$$

If $h \geq|Y|+k+2$, then this number is negative, a contradiction to the selfsufficiency of $A$.

Now (1) and (3) imply the assertion for the case $0<k$. We assume $k=0$. If for all $2 \leq j \leq h$ we have $V_{j} \neq \emptyset$, then by (3) we obtain again the assertion. Hence we can assume w.l.o.g. that $V_{2}=\emptyset$. Use (S2). We show that this is impossible. First we have to prove:

(4) If $k=0$ and $V_{2}=\emptyset$, then $Y \subseteq A$. 
$V_{2}=\emptyset$ implies that $X_{2}^{-}$is linearly independent over $A$. By (S1) the $\Phi_{j}^{2}=$ $\sum_{1 \leq t \leq r}\left(y_{t}^{j} \wedge z_{t}^{2}\right)$ for $1 \leq j \leq r$ generate $N\left(\left\langle Y X_{2}^{-}\right\rangle\right)$over $N(\langle Y\rangle)$ freely. If some $y_{t}^{j}$ $(1 \leq t \leq r)$ is not in $A$, then it is impossible to write $\Phi_{j}^{2}$ as a linear combination of $\Delta_{1}, \ldots, \Delta_{n}$ modulo $\wedge^{2} A$. Hence (4) is shown.

Now (4) implies that $\left\langle Y X_{2}^{-}\right\rangle$is a minimal extension of $\langle Y\rangle \subseteq A$. Then $\Phi_{1}^{2}, \ldots, \Phi_{r}^{2}$ are linearly independent over $\wedge^{2} A$. We have $\left\langle A X_{2}^{-}\right\rangle=B$ and $r=n$. But in the assumptions of the lemma we have excluded such a basis $\Phi_{1}^{2}, \ldots, \Phi_{n}^{2}$ of $N(B)$ over $N(A)$ that only uses basic commutators $a_{i} \wedge b_{i}$ where $a_{i} \in A$ and $b_{1}, \ldots, b_{n}$ is a basis of $B$ over $A$. Hence we have the desired contradiction.

Lemma 8.4. $\overline{\Sigma^{*}}$ is CM-trivial.

Proof. Let $M$ be a saturated model of $\overline{\Sigma^{*}}$. Then Lemma 8.1 gives a model $G$ of $\Sigma^{*}$ defined in $M . G$ is saturated, and $M$ is the induced structure on $\bar{G}$. We also use $M$ to denote the corresponding $\mathcal{S}$-structure. The following proof uses the fact that $M$ is strongly minimal, that $a c l=c l$, and that independence in the sense of forking is geometrical independence.

Assume $B_{1}, B_{2}, A$, and $E$ are algebraically closed sets in $M^{e q}$. Let $\overline{B_{i}}$ be $\operatorname{acl}\left(B_{i} E\right)$. Furthermore assume that the following conditions are fulfilled:

i) $\operatorname{acl}\left(B_{1}+B_{2}\right) \cap \overline{B_{i}}=B_{i}$.

ii) $B_{i} \cap E=A$.

iii) $B_{1}$ and $B_{2}$ are independent over $E$.

We have to show that $B_{1}$ and $B_{2}$ are independent over $A$.

Since $\overline{\Sigma^{*}}$ has weak elimination of imaginaries, every algebraically closed set is the algebraic closure of an algebraically closed set in the real sort. Hence we can work in the model $M$ instead of $M^{e q}$. By iii)

$$
\overline{B_{1}}+\overline{B_{2}}=\overline{B_{1}} \star_{E} \overline{B_{2}}
$$

and $\overline{B_{1}}+\overline{B_{2}}$ is selfsufficient in $M$.

Since $\overline{B_{1}} \cap \overline{B_{2}}=E$ we obtain $B_{1} \cap B_{2} \subseteq E$. Hence $B_{i} \cap E=A$ implies $B_{1} \cap B_{2}=A$. It is sufficient to show that $B_{1}+B_{2}$ is selfsufficient and

$$
B_{1}+B_{2}=B_{1} \star_{A} B_{2} \text {. }
$$

W.l.o.g. we assume that all sets considered have finite geometrical dimension.

(5) $B_{1}+B_{2}$ is selfsufficient .

Indeed, we know that $\left(\overline{B_{1}}+\overline{B_{2}}\right) \cap \operatorname{acl}\left(B_{1}+B_{2}\right)$ is selfsufficient. Hence we can work in this selfsufficient subspace. If $B_{1}+B_{2}$ was not selfsufficient, then there would exist an extension $D$ of $B_{1}+B_{2}$ such that

$$
\begin{aligned}
& \text { the linear dimension of } D \text { over }\left(B_{1}+B_{2}\right) \text { is finite, } \\
& D \subseteq \operatorname{acl}\left(B_{1}+B_{2}\right) \cap\left(\overline{B_{1}}+\overline{B_{2}}\right) \text {, and } \\
& \delta(D)<\delta\left(B_{1}+B_{2}\right)
\end{aligned}
$$

Since $\operatorname{acl}\left(B_{1}+B_{2}\right) \cap \overline{B_{i}}=B_{i}, D$ has a linear basis $\left\{u_{1}+v_{1}, \ldots, u_{n}+v_{n}\right\}$ over $B_{1}+B_{2}$, where $u_{1}, \ldots, u_{n} \in \overline{B_{1}}$ are linearly independent over $B_{1}+E$ and $v_{1}, \ldots, v_{n} \in \overline{B_{2}}$ are linearly independent over $B_{2}+E$ (Lemma 4.2).

Since $\delta(D)<\delta\left(B_{1}+B_{2}\right)$, there are $m>n$ many relation $\Phi_{1}, \ldots, \Phi_{m}$ in $N(D)$ that are linearly independent over $\wedge^{2}\left(B_{1}+B_{2}\right)$. By Lemma 4.3 there are $a_{i}^{j}$, 
$\Psi_{j}^{1} \in \wedge^{2} B_{1}, \Psi_{j}^{2} \in \wedge^{2} B_{2}$, and $I_{j} \in \wedge^{2} E$ for $1 \leq j \leq m, 1 \leq i \leq n$ such that

$$
\begin{gathered}
\Phi_{j}=\sum_{1 \leq i \leq n}\left(a_{i}^{j} \wedge\left(u_{i}+v_{i}\right)\right)+\Psi_{j}^{1}+\Psi_{j}^{2}, \\
\Phi_{j}^{1}=\sum_{1 \leq i \leq n}\left(a_{i}^{j} \wedge u_{i}\right)+\Psi_{j}^{1}+I_{j} \in N\left(\overline{B_{1}}\right), \\
\Phi_{j}^{2}=\sum_{1 \leq i \leq n}\left(a_{i}^{j} \wedge v_{i}\right)+\Psi_{j}^{2}-I_{j} \in N\left(\overline{B_{2}}\right) .
\end{gathered}
$$

Note that it follows that $A \neq \emptyset$. The linear independence of $\Phi_{1}, \ldots, \Phi_{m}$ over $\wedge^{2}\left(B_{1}+B_{2}\right)$ implies that the $\left(\sum_{1 \leq i \leq n} a_{i}^{j} \wedge u_{i}\right)$ for $1 \leq j \leq m$ are linearly independent over $\wedge^{2}\left(B_{1}+E\right)$ and the $\left(\sum_{1 \leq i \leq n} a_{i}^{j} \wedge v_{i}\right)$ for $1 \leq j \leq m$ are linearly independent over $\wedge^{2}\left(B_{2}+E\right)$. Hence $\Phi_{1}^{1}, \ldots, \Phi_{m}^{1}$ are linearly independent over $\wedge^{2}\left(B_{1}+E\right)$ and $\Phi_{1}^{2}, \ldots, \Phi_{m}^{2}$ are linearly independent over $\wedge^{2}\left(B_{2}+E\right)$. W.l.o.g. we can assume that there is some $m_{0} \leq m$ such that $I_{j}=0$ for $1 \leq j<m_{0}$ and $I_{m_{0}}, \ldots, I_{m}$ are linearly independent over $\wedge^{2} A$. Then $m_{0} \leq n \leq m$, since otherwise $\delta\left(B_{1}+\right.$ $\left.\left\langle u_{1}, \ldots, u_{n}\right\rangle\right) \leq \delta\left(B_{1}\right)$ would imply $u_{1}, \ldots, u_{n} \in B_{1}$, a contradiction. Assume that $D$ is chosen of minimal size over $B_{1}+B_{2}$. That means $n$ is minimal. Let $B_{1}^{\prime} \subseteq B_{1}$, $E^{\prime} \subseteq E$, and $A^{\prime} \subseteq A$ be finite selfsufficient subspaces such that $\delta\left(B_{1}^{\prime}\right)=d\left(B_{1}\right)$, $\delta\left(E^{\prime}\right)=d(E), \delta\left(\overline{A^{\prime}}\right)=d(A), I_{j} \in \wedge^{2} E^{\prime}, \Psi_{j}^{1} \in \wedge^{2} B_{1}^{\prime}($ for $1 \leq j \leq m, 1 \leq i \leq n)$, and $E^{\prime} \cap A=B_{1}^{\prime} \cap A=A^{\prime}=E^{\prime} \cap B_{1}^{\prime}$.

Let $\left(E^{\prime}, N\left(E^{\prime}\right)\right)$ be the $\mathcal{S}$-structure corresponding to $E^{\prime}$. We define a finite $\mathcal{S}$ structure $C$ that extends $E^{\prime}$. We prove that $C$ is an algebraic extension of $E^{\prime}$ that satisfies $(\Sigma 3)$ and $(\Sigma 4) . C$ is freely generated by $c_{1}, \ldots, c_{n}$ over $E^{\prime}$, and $N(C)$ is freely generated over $N\left(E^{\prime}\right)$ by $\Delta_{1}, \ldots, \Delta_{n}$, where $\Delta_{j}=\left(\sum_{1 \leq i \leq n} a_{i}^{j} \wedge c_{i}\right)+I_{j}$ is obtained from $\Phi_{j}^{1}(1 \leq j \leq n)$ above.

(6) $\delta(C)=\delta\left(E^{\prime}\right)$ and $\mathrm{C}$ satisfies $(\Sigma 3)$.

We prove (6). The linear independence of $\Phi_{1}^{1}, \ldots, \Phi_{n}^{1}$ over $\wedge^{2}\left(E+B_{1}\right)$ implies that $\Delta_{1}, \ldots, \Delta_{n}$ are linearly independent over $\wedge^{2} E^{\prime}$. Hence $\delta(C)=\delta\left(E^{\prime}\right)$. For the proof of (6) we show for every subspace $K$ of $C$ :

(7) $\delta(K) \geq \delta\left(K \cap E^{\prime}\right)$

If $\operatorname{dim}(K)-\operatorname{dim}\left(K \cap E^{\prime}\right)=n$, then (7) is clear. Assume $\delta(K)<\delta\left(K \cap E^{\prime}\right)$. We show a contradiction. Let $K$ be freely generated by $d_{1}, \ldots, d_{l}$ over $K \cap E^{\prime}$. Then $l<n$ and there are $l+1$ relations $\Theta_{1}, \ldots, \Theta_{l+1}$ in $N(K)$ linearly independent over $\wedge^{2}\left(K \cap E^{\prime}\right)$. Note that every $\Theta_{j}$ is a linear combination of $\Delta_{1}, \ldots, \Delta_{n}$ modulo $N\left(E^{\prime}\right)$. Every $d_{i}$ is a linear combination $d_{i}^{+}$of $c_{1}, \ldots, c_{n}$ modulo $E^{\prime}$. These $d_{i}^{+}$are linearly independent over $E^{\prime}$. If we consider the corresponding linear combinations $w_{i}^{+}$of $u_{1}+v_{1}, \ldots, u_{n}+v_{n}$, then

$$
\delta\left(B_{1}+B_{2}+\left\langle w_{1}^{+}, \ldots, w_{l}^{+}\right\rangle\right)<\delta\left(B_{1}+B_{2}\right),
$$

a contradiction to the minimality of $n$. This proves (7).

From (7) we get $\delta(K) \geq 3$, if $\operatorname{dim}\left(K \cap E^{\prime}\right) \geq 3$, because $(\Sigma 3)$ is true for $E^{\prime}$. Let us assume that $\operatorname{dim}\left(K \cap E^{\prime}\right)<3$. Then

$$
\delta\left(K \cap E^{\prime}\right)=\operatorname{dim}\left(K \cap E^{\prime}\right) \quad \text { and } \quad \operatorname{dim}\left(K \cap A^{\prime}\right)<3 .
$$

We discuss the three possible cases for $\operatorname{dim}\left(K \cap A^{\prime}\right)$. 
If $\operatorname{dim}\left(K \cap A^{\prime}\right)=0$, then no nontrivial linear combination of $\Delta_{1}, \ldots, \Delta_{n}$ is in $N(K)$ modulo $N\left(E^{\prime}\right)$, since the $\left(\sum_{1 \leq i \leq n} a_{i}^{j} \wedge c_{i}\right)$ 's are linearly independent. Then $\delta(K)=\delta\left(K \cap E^{\prime}\right)+\operatorname{dim}(K)-\operatorname{dim}\left(K \cap E^{\prime}\right)=\operatorname{dim}(K)$, as desired.

If $\operatorname{dim}\left(K \cap A^{\prime}\right)=1$, then either $N(K)=N\left(K \cap E^{\prime}\right)=0$ or there is a relation of the form $\Delta=a \wedge d+I$ in $N(K)$, where $\langle a\rangle=K \cap A^{\prime}, I \in \wedge^{2}\left(E^{\prime} \cap K\right)$ and $d=c$ modulo $E^{\prime}$, where $c$ is a nontrivial linear combination of $c_{1}, \ldots, c_{n}$. $\Delta$ has this form because w.l.o.g. it is a linear combination of the $\Delta_{i}$ 's. We can rewrite $\Delta$ as $a \wedge c+I^{\prime}$, where $I^{\prime} \in \wedge^{2} E^{\prime}$. We find a linear combination $\Psi \in N\left(E+B_{1}+\left\langle\left\{u_{1}, \ldots, u_{n}\right\}\right\rangle\right)$ of $\Phi_{1}^{1}, \ldots, \Phi_{n}^{1}$ corresponding to $\Delta$. That means that in the linear combination $\Delta$ we replace every $\Delta_{i}$ by the corresponding $\Phi_{i}^{1}$. We get that $\Psi$ is of the form $a \wedge u+\Psi^{1}+I^{\prime}$, where $\Psi^{1} \in \wedge^{2} B_{1}$, and $u$ is a nontrivial combination of $u_{1}, \ldots, u_{n}$. Since $E$ is algebraically closed, there is some $e \in E$ with $a \wedge e=I^{\prime}$ (use $(\Sigma 2)$ ). Since $B_{1}$ is algebraically closed, there is some $b \in B_{1}$ with $a \wedge b=\Psi^{1}$. Hence $a \wedge(u+e+b) \in N\left(E+B_{1}\right)$. By $(\Sigma 3)$ for $M$ we obtain $u+e+b=r a$. Then $u=r a-e-b \in E+B_{1}$. This is a contradiction, since $u_{1}, \ldots, u_{n}$ are linearly independent over $E+B_{1}$.

There remains the case $\operatorname{dim}\left(K \cap A^{\prime}\right)=2$. Then $K \cap A^{\prime}=K \cap E^{\prime}$. If $\operatorname{dim}(K)=2$, then $K=K \cap A^{\prime}$ and $\delta(K)=\operatorname{dim}(K)=2$, as desired. Otherwise $\operatorname{dim}(K)=r+2>$ 2. We suppose $\delta(K)<3$ and derive a contradiction. By $(7) \delta(K)=\delta\left(K \cap A^{\prime}\right)=2$. Hence there are $r$ linear combinations $\Theta_{1}, \ldots, \Theta_{r}$ in $N(K)$ linearly independent over $\wedge^{2}\left(K \cap A^{\prime}\right)$. There are linear combinations $b_{1}, \ldots, b_{r}$ of $c_{1}, \ldots, c_{n}$ that are linearly independent and elements $e_{1}, \ldots, e_{r} \in E^{\prime}$ such that

$$
K=\left(K \cap A^{\prime}\right)+\left\langle b_{1}+e_{1}, \ldots, b_{r}+e_{r}\right\rangle
$$

Since we can replace $c_{1}, \ldots, c_{n}$ by any other basis of $C$ over $E^{\prime}$, we can assume w.l.o.g. that $c_{i}=b_{i}$ and $e_{i}=0$ for $1 \leq i \leq r$. Since every $\Theta_{i}$ has to be a nontrivial linear combination of the $\Delta_{j}$ modulo $N\left(E^{\prime}\right)$ we can assume w.l.o.g that $\Theta_{1}=\Delta_{1}, \ldots, \Theta_{r}=\Delta_{r} . \Delta_{i} \in N(K)$ implies $I_{i} \in \wedge^{2}\left(K \cap A^{\prime}\right)$. Hence $\Phi_{1}^{1}, \ldots, \Phi_{r}^{1} \in$ $N\left(B_{1}+\left\langle u_{1}, \ldots, u_{r}\right\rangle\right)$ are linearly independent over $N\left(B_{1}\right)$. Hence $u_{1}, \ldots, u_{n} \in B_{1}$, a contradiction. (6) is shown.

If $C$ is not a minimal extension of $E^{\prime}$, then there are $E^{\prime}=C_{0} \subseteq C_{1} \subseteq \ldots \subseteq C_{s}=$ $C$ such that $C_{i+1}$ is a minimal extension of $C_{i}$ for $0 \leq i<s$. Every $C_{i+1}$ is freely generated by some $d_{1}, \ldots, d_{l}$ over $C_{i}$, and $N\left(C_{i+1}\right)$ is freely generated over $N\left(C_{i}\right)$ by $l$ relations of the form $\left(\sum_{1 \leq i \leq l} a_{i}^{j} \wedge d_{i}\right)+J_{j}$, where $a_{i}^{j} \in A^{\prime}$ and $J_{j} \in \wedge^{2} C_{i}$. These relations are linear combinations of $\Delta_{1}, \ldots, \Delta_{n}$ modulo $N\left(E^{\prime}\right)$. We cannot choose $d_{1}, \ldots, d_{l}$ in such a way that $J_{1}=\ldots=J_{l}=0$. Otherwise we could choose the corresponding $u_{1}, \ldots, u_{l}$ in $B_{1}$, a contradiction. Hence we can apply Lemma 8.3 to every $C_{i+1}$ over $C_{i}$. We have $(\Sigma 4)$ for all $C_{i}$ :

(8) $C$ satisfies $(\Sigma 4)$.

Now we can apply axiom $(\Sigma 5)$ successively to every $C_{i+1}$ over $C_{i}$. Since $M$ was chosen saturated, there is a selfsufficient image of $C$ in $M$. We denote it again by $C$. Since $E$ was algebraically closed, $C \subseteq E$. Hence we have $c_{1}, \ldots, c_{n}$ in $E$ such that for $1 \leq j \leq n$

$$
\left(\sum_{1 \leq i \leq n} a_{i}^{j} \wedge c_{i}\right)+I_{j} \in N(E) .
$$


It follows that

$$
\left(\sum_{1 \leq i \leq n} a_{i}^{j} \wedge\left(u_{i}-c_{i}\right)\right)+\Psi_{j}^{1} \in N\left(\overline{B_{1}}\right) .
$$

Since these relations are linearly independent over $N\left(B_{1}^{\prime}\right), a_{i}^{j} \in A^{\prime}$, and $\Psi_{j}^{1} \in \wedge^{2} B_{1}^{\prime}$, we obtain that $u_{i}-c_{i}$ are in the algebraic closure of $B_{1}^{\prime}$, i.e., in $B_{1}$. We have shown that $u_{i}=c_{i}+\left(u_{i}-c_{i}\right) \in E+B_{1}$, a contradiction. Hence $B_{1}+B_{2}$ is selfsufficient, as desired.

It remains to show that $B_{1}+B_{2}=B_{1} \star_{A} B_{2}$. This follows from:

(9) $\delta\left(B_{1}+B_{2}\right)=\delta\left(B_{1}\right)+\delta\left(B_{2}\right)-\delta(A)$.

By Lemma 3.4 we have $\delta\left(B_{1}+B_{2}\right) \leq \delta\left(B_{1}\right)+\delta\left(B_{2}\right)-\delta(A)$. To show equality we consider some $\Phi$ in $N\left(B_{1}+B_{2}\right)$. Since we work in $\overline{B_{1}} \star_{E} \overline{B_{2}}$ we obtain by Lemma $4.3 \Phi=\Phi^{1}+I+\Phi^{2}-I$, where $\Phi^{1}+I \in N\left(\overline{B_{1}}\right), \Phi^{2}-I \in N\left(\overline{B_{2}}\right), \Phi^{1} \in \wedge^{2} B_{1}$, $\Phi^{2} \in \wedge^{2} B_{2}$, and $I \in \wedge^{2} E$. Let $a$ be one of the fixed constants in $M$. By $(\Sigma 2)$ there is some $e \in E$ such that $a \wedge e+I \in N(E)$. Then $\Phi^{1}-a \wedge e \in N\left(\overline{B_{1}}\right)$. Since $a \in B_{1}$, $\Phi^{1} \in \wedge^{2} B_{1}$, and $B_{1}$ is algebraically closed we obtain $e \in B_{1}$. On the other hand $\Phi^{2}+a \wedge e \in N\left(\overline{B_{2}}\right)$. Similarly as above we obtain $e \in B_{2}$. Hence $e \in B_{1} \cap B_{2}=A$. Then $\Phi=\Phi^{1}-a \wedge e+\Phi^{2}+a \wedge e$, where $\Phi^{1}-a \wedge e \in N\left(B_{1}\right)$ and $\Phi^{2}+a \wedge e \in N\left(B_{2}\right)$, as desired. CM-triviality of $\overline{\Sigma^{*}}$ is shown.

Lemma 8.2 and Lemma 8.4 imply

Theorem 8.5. $\Sigma$ is CM-trivial.

CM-triviality implies the non-interpretability of a field and of a simple group in the case of finite Morley rank, as proved by A. Pillay [21]. Hence from Theorem 8.5 we conclude:

Theorem 8.6. No field is interpretable in $\Sigma$.

Together with Theorem 2.1 we obtain:

Corollary 8.7. Every group interpretable in $\Sigma$ is a direct sum of a group that is nilpotent by finite and of bounded exponent, and an abelian group.

Furthermore we have proved in [6]:

Theorem 8.8. Every group interpretable in $\Sigma$ is nilpotent by finite and of bounded exponent.

\section{REFERENCES}

1. J. T. Baldwin and A. H. Lachlan On strongly minimal sets, JSL 36 (1971), 79-96. MR 44:3851

2. A. Baudisch, Decidability and stability of free nilpotent Lie algebras and free nilpotent pgroups of finite exponent, Ann. of Math. Logic 23 (1982), 1-25. MR 84e:03019

3. A. Baudisch, On Lascar rank in non-multidimensional $\omega$-stable theories, Logic Colloquium ' 85 in Paris, North-Holland, Amsterdam 1987, 33-51. MR 88k:03068

4. A. Baudisch, Classification and interpretation, JSL 54 (1989), 138-159. MR 90c:03026

5. A. Baudisch, On superstable groups, J. London Math. Soc. 42 (1990), 452-464. MR 92e:03044

6. A. Baudisch, A new uncountably categorical pure group, Preprint Nr. A 93-40 Freie Universität Berlin 1993.

7. O. V. Belegradek, On groups of finite Morley rank, Abstracts of the $8^{\text {th }}$ International Congress of Logic, Methodology, and Philosophy of Science, Moscow, 1987, 100.

8. Ch. Berline and D. Lascar, Superstable groups, Ann. Pure and Appl. Logic 30 (1986), 1-43. MR 87k:03028

9. R. Fraissé, Sur l'extension aux relations de quelques propriétés des ordres, Ann. Sci. École Norm. Sup. 71 (1954), 361-388. MR 16:1006b 
10. J. B. Goode, Hrushovski's geometries, Proceedings of the $7^{\text {th }}$ Easter Conference on Model Theory 1989, Seminarberichte der Humboldt-Universität zu Berlin Nr. 104, 106-117. CMP 1990:9

11. C. Grünenwald and F. Haug, On stable torsion-free nilpotent groups, Arch. Math. Logic 32 (1993), 451-462. MR 95e:03105

12. E. Hrushovski, A new strongly minimal set, Ann. Pure Appl. Logic 62 (1993), 147-166. MR 94d:03064

13. E. Hrushovski and A. Pillay, Weakly normal groups, Logic Colloquium' 85 in Paris, edited by the Paris Logic Group, North-Holland, Amsterdam, 1987, 233-244. MR 88e:03051

14. S. Lang, Algebra, Addison-Wesley, 1965. MR 33:5416

15. M. D. Morley, Categoricity in power, Trans. A.M.S. 114 (1965), 514 -538. MR 31:58

16. A. Nesin, Poly-separated and $\omega$-stable nilpotent groups, J. Symbolic Logic 56 (1991), 915-931. MR 93b:03049

17. B. Poizat, Cours de théorie de modèles, Nur al-Mantiq wal Ma'rifah, Lyon, 1985. MR 87f:03084

18. B. Poizat, Groupes stables, Nur al-Mantiq wal Ma'rifah, Lyon, 1987. MR 89b:03056

19. B. Poizat, Une théorie de Galois imaginaire, JSL 48 (1983), 1151-1176. MR 85e:03083

20. A. Pillay, Some remarks on modular regular types, JSL 56 (1991), 1003-1011. MR 92g:03053

21. A. Pillay The geometry of forking and groups of finite Morley rank, preprint 1994.

22. S. Shelah, Classification theory and the number of non-isomorphic models (Second Edition), North-Holland, Amsterdam, 1990. MR 91k:03085

23. B. Zil'ber, Groups and rings with categorical theories (Russian), Fund. Math. 95 (1977), 173188; English transl., Amer. Math. Soc. Transl. (2) 149 (1991), 1-16. MR 56:118

24. B. Zil'ber, Some model theory of simple algebraic groups over algebraically closed fields, Colloquium Math. 48 (1984), 173-180. MR 86f:03055

25. B. Zil'ber, The strucutre of models of uncountably categorical theories, Proc. Internat. Congr. Math. (Warsaw, 1983), Vol. 1, PWN, Warsaw, 1984, pp. 359-368. MR 87d:03093b

26. B. Zil'ber, Uncountably categorical nilpotent groups and Lie algebras, Soviet Math. (Iz. VUZ) 26 (1982), 98 -99.

2. Mathematisches Institut, Freie Universität Berlin, Arnimallee 3, 14195 Berlin Current address: Fachbereich Mathematik, Humboldt-Universität zu Berlin, Unter den Linden 6, 10099 Berlin, Germany

E-mail address: baudisch@mathematik.hu-berlin.de 